\title{
A Review of Neotropical Myxomycetes (1828-2008)
}

\author{
by \\ Carlos Lado \& Diana Wrigley de Basanta \\ Real Jardín Botánico, CSIC, Plaza de Murillo 2, 28014 Madrid, Spain. lado@rjb.csic.es
}

\begin{abstract}
Lado, C. \& Wrigley de Basanta, D. 2008. A Review of Neotropical Myxomycetes (1828-2008). Anales Jard. Bot. Madrid 65(2): 211-254.

A synthesis of the accumulated knowledge on myxomycetes recorded from the Neotropical region is presented in this paper. The biodiversity of these microorganisms in the Neotropics has been underestimated, and this paper shows that half the known species in the world have been recorded from the region. The monograph by M.L. Farr, for the series Flora Neotropica, published in 1976, has been taken as a baseline. The records produced after this date, some older obscure records, and data from recently published catalogues, monographs and other papers have been incorporated. The information is presented in a table format by species and countries. Species names are listed with synonyms that have been used in Neotropical literature and nomenclature has been updated. A comprehensive list of references by country has been included. A characteristic assemblage of myxomycetes from the Neotropics has been identified. The richness of myxobiota in different countries has been evaluated, and gaps in current information and unexplored areas have become evident from the results. Use of the compiled information to direct conservation plans, and to serve as a starting point to establish and develop future strategies for the study of myxomycetes in this area of the world, is discussed. The importance of prioritizing this research on microorganismal biodiversity, in view of accelerated habitat destruction, is stressed.
\end{abstract}

Keywords: biodiversity, microorganisms, protists, Mycetozoa, tropics, geographical distribution, catalogue, Central America, Caribbean, South America.

\section{Introduction}

The biodiversity of microorganisms is a topic that is becoming increasingly important since they are the very basis of ecosystems. Myxomycetes are eukaryotic microorganisms, with unicellular and coenocytic

\section{Resumen}

Lado, C. \& Wrigley de Basanta, D. 2008. Revisión de los Myxomycetes del Neotrópico (1828-2008). Anales Jard. Bot. Madrid 65(2): 211-254 (en inglés).

Se realiza una síntesis sobre el conocimiento actual de los Myxomycetes en el Neotrópico. La biodiversidad de estos microorganismos en la región neotropical ha sido subestimada, pero este trabajo demuestra que la mitad de las especies conocidas en el mundo se han citado de esta región. La monografía que M.L. Farr publicó en 1976, para la serie Flora Neotrópica, se ha tomado como punto de partida para la realización de este trabajo. A ella se han incorporado las citas publicadas después de esta fecha, algunas más antiguas pero raras, y datos de catálogos, monografías y otros trabajos recientes. La información se presenta en una tabla de doble entrada, por orden alfabético de especies y por países. La nomenclatura de las especies se ha actualizado y se han añadido los sinónimos con los que han sido citadas en la bibliografía neotropical. También se incluye una lista de referencias bibliográficas por países. Se ha podido identificar un conjunto de especies de Myxomycetes que, por su abundancia de citas en los países neotropicales, parecen características de la región. Se evalúa, por países, la riqueza de su mixobiota y se ponen de manifiesto la falta de información y los escasos estudios que se han llevado a cabo en determinados territorios de esta región biogeográfica. Se discute y comenta el uso que se puede dar a esta información recopilada, como punto de partida para establecer y desarrollar estrategias de estudio sobre los Myxomycetes en esta parte del mundo. Por último, se llama la atención sobre la importancia y prioridad que se debe dar a la investigación sobre biodiversidad de microorganismos, a la hora de valorar la acelerada destrucción de hábitat.

Palabras clave: biodiversidad, microorganismos, protistas, Mycetozoa, trópicos, distribución geográfica, catálogo, América Central, Caribe, América del Sur.

phagotrophic phases. They inhabit all terrestrial ecosystems, feeding on bacteria and other microorganisms, in and on plant parts and plant remains. Some are known to be associated with specific ecosystems, while others are more cosmopolitan, and research into their diversity and their specific relation- 
ships within certain ecosystems is an emerging focus of recent research. This is especially critical in areas like the Neotropics, where rapid habitat loss endangers all components of the various biomes. The Neotropical region is one of the biogeographical regions with the highest biodiversity in the world. Estimates by Davis \& al. (1997), show that more than 70.000 endemic plant species exist in the Neotropics, and the Tropical Andean region alone, contains about a sixth of all plant life in less than $1 \%$ of the world's land area. More than a third of the centres of plant diversity and endemisms recognized by Davis \& al. (1997) and eight of the designated biodiversity "hot spots", where "exceptional concentrations of endemic species are undergoing exceptional loss of habitat" (Myers \& al., 2000), are located in this area (Mittermeier \& al., 2004). In contrast, the knowledge of myxomycetes of the Neotropics is far from complete. The first record of myxomycetes in the Neotropics was in 1828, from Chile (Bertero, 1828). In the $19^{\text {th }}$ Century there were various publications from the region. Farr (1976), included these in a monograph published in the series Flora Neotropica, in which she compiled all the information available up to the year 1975. In this monograph, 250 of the almost 900 myxomycete species known in the world (Lado, 2008), were reported for this region. This is about the same as in single, possibly less diverse, but well studied countries, like the United Kingdom, the Netherlands, Ireland, France or Spain, which have 250-350 species recorded (Nannenga-Bremekamp, 1991; Lado, 1994; Ing, 1999).

Several research projects on Neotropical myxomycetes, supported by science foundations or research institutions from different countries such as Spain, USA, Brazil or Mexico, have been developed over the last decade and some are currently in progress. The objectives and outcomes of these projects are different but complementary. Some are devoted to the study of pristine tropical forest (Novozhilov \& al., 2000; Schnittler \& al., 2002; Lado \& al., 2003; Stephenson \& al., 2003), others to special microhabitats (Schnittler, 2001; Schnittler \& Stephenson, 2002a; Maimoni-Rodella \& Cavalcanti, 2006; Wrigley de Basanta \& al., 2008), others to the knowledge of the myxobiota of specific environments (Mosquera \& al., 2000b; Lado \& al., 2007b; Estrada-Torres \& al., in press), and others make inventories of regions (Hochgesand \& Gottsberger, 1996; Pando, 1997; Lizárraga \& al., 1997; Estrada-Torres \& al., 2001; Putzke, 2002; Cavalcanti, 2002; MaimoniRodella, 2002). Additionally, some recent cheklists and catalogues of Myxomycetes, from regions or countries such as Colombia (Uribe-Meléndez, 1995), French Guayana (Courtecuisse \& al., 1996), Brazil (Putzke, 1996), Mexico (Ogata \& al., 1994; Illana \& al., 2000; Moreno \& al., 2007), the Caribbean (Minter
\& al., 2001) or Argentina (Crespo \& Lugo, 2003), have been published, and new species or genera have been described from the region (Hochgesand \& Gottsberger, 1989; Lado \& al., 1999a, 2007b; Mosquera \& al., 2003; Estrada-Torres \& al., 2001, 2003). All these projects, and their resulting publications have provided, in a short time, an important and valuable body of information, on the myxobiota of this bioregion, but much of the data is dispersed and, in some cases, is difficult to obtain.

The objective of this paper is to compile and synthesize all the accumulated information on the presence of Myxomycetes, in this part of the world. Apart from providing an up to date view of the knowledge of myxomycetes from the Neotropics, it can be used to show gaps of information, and areas currently unexplored. In addition, it can be used to evaluate the richness of the myxobiota in different countries, and so provide information for future conservation and protection plans, and also serve as a starting point to establish and develop future strategies for the study of myxomycetes in this area of the world.

\section{Geographic area covered}

The geographic area covered by this review includes all of the American territories between the Tropic of Cancer and the Tropic of Capricorn, and encompasses all the Mesoamerica and Caribbean bioregions as well as South America. We consider whole countries in a political sense, even when the limits of all the territory of the country are not included between the limits of the Neotropical region in a strict sense, as is the case of Mexico, Argentina and Chile. Uruguay, the only country out of the limits of the tropics, but with subtropical features, is also included. With these criteria the paper includes all of the territories between Mexico (Fig. 1), as the northern limit, and Tierra del Fuego (Fig. 2), as the southern limit.

The area has been divided, for practical reasons, into thirty regions, which largely conform to political circumscriptions. But the designations of geographical entities do not imply the expression of any opinion whatsoever concerning the legal status of any country, territory or area, or of its authorities, or concerning the delimitation of its frontiers or boundaries. The countries are designated with the three-letter code of Botanical Countries established by Brummitt (2001) in the World Geographical Scheme for Recording Plant Distributions. Some exceptions were made, to enable an easier interpretation of the data, and so a single code is used for all the continental territory of Mexico (MEX) as well as its Pacific and Caribbean Islands, and for all of the territories of Brazil (BZI). Those of Argentina (AGA) and Chile (CLI), include each country's area of Tierra del 


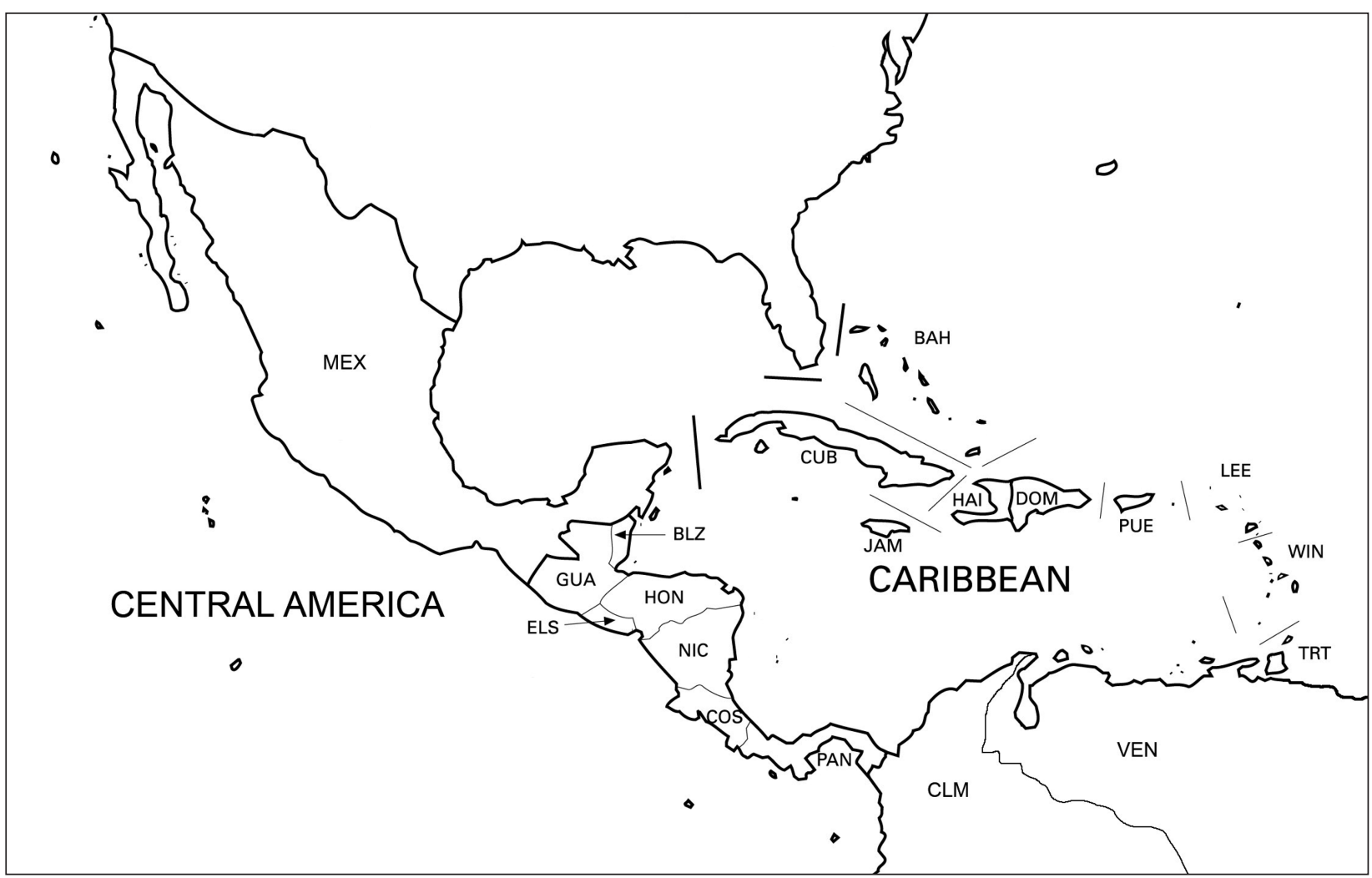

Fig. 1. Central America and the Caribbean.

Fuego. Tiny enclaves of one country in another have been ignored, and geographical disjunctions such as major or remote islands have been included in their countries of political dependence. According to these criteria, the Desventurados Islands and Juan Fernandez Islands are also included in Chile (CLI), the Galapagos Islands are included in Ecuador (ECU), and the Fernando de Noronha and Trindade Islands in Brazil (BZI).

Due to the large number of islands and to avoid too much division of the territory, the Leeward Islands of the Caribbean bioregion, such as Anguilla, AntiguaBarbuda, Guadalupe, Montserrat or the American Virgin Islands, have been considered as one territory (LEE). The same criteria have been applied to the Bahama Islands (BAH) and the Windward Islands (WIN), that include Barbados, Dominica, Grenada, St. Lucia, Martinique and St. Vincent. The little islands making up the Central American Pacific Islands such as Cocos, Coiba and Malpelo, are included in the countries of political dependence (Costa Rica, Panama or Colombia), as have the Southwest Caribbean Islands of Colombian, Honduran and Nicaraguan Islands. The Dutch territories of Aruba, Bonaire and Curaçao Islands, are included in Venezuela as well as the Venezuelan Antilles. The Turks and Caicos Islands have been included with the Bahamas, and the
Cayman Islands jointly with Cuba. Abbreviations used herein are listed and shown on figs. 1-2.

\section{Central America}

MEX Mexico (Guadalupe Island, Rocas Alijos Islands and Revillagigedo Islands included)

BLZ Belize

GUA Guatemala

HON Honduras (Honduran Caribbean Islands included)

ELS El Salvador

NIC Nicaragua (Nicaraguan Caribbean Islands included)

COS Costa Rica (Cocos Island included)

PAN Panama (Coiba Island included)

\section{Caribbean}

BAH Bahamas (Turks and Caicos Islands included)

CUB Cuba (Cayman Islands included)

JAM Jamaica

HAI Haiti (Navassa Island included)

DOM Dominican Republic

PUE Puerto Rico

LEE Leeward Islands (Antigua-Barbuda, Anguilla, Aves Island, British Virgin Islands, Guadeloupe, Montserrat, Netherlands Leeward Islands, St. Kitts-Nevis, St. Martin-St. Barthélémy and Virgin Islands)

WIN Windward Islands (Barbados, Dominica, Grenada, Martinique, St. Lucia, St. Vincent)

TRT Trinidad and Tobago 


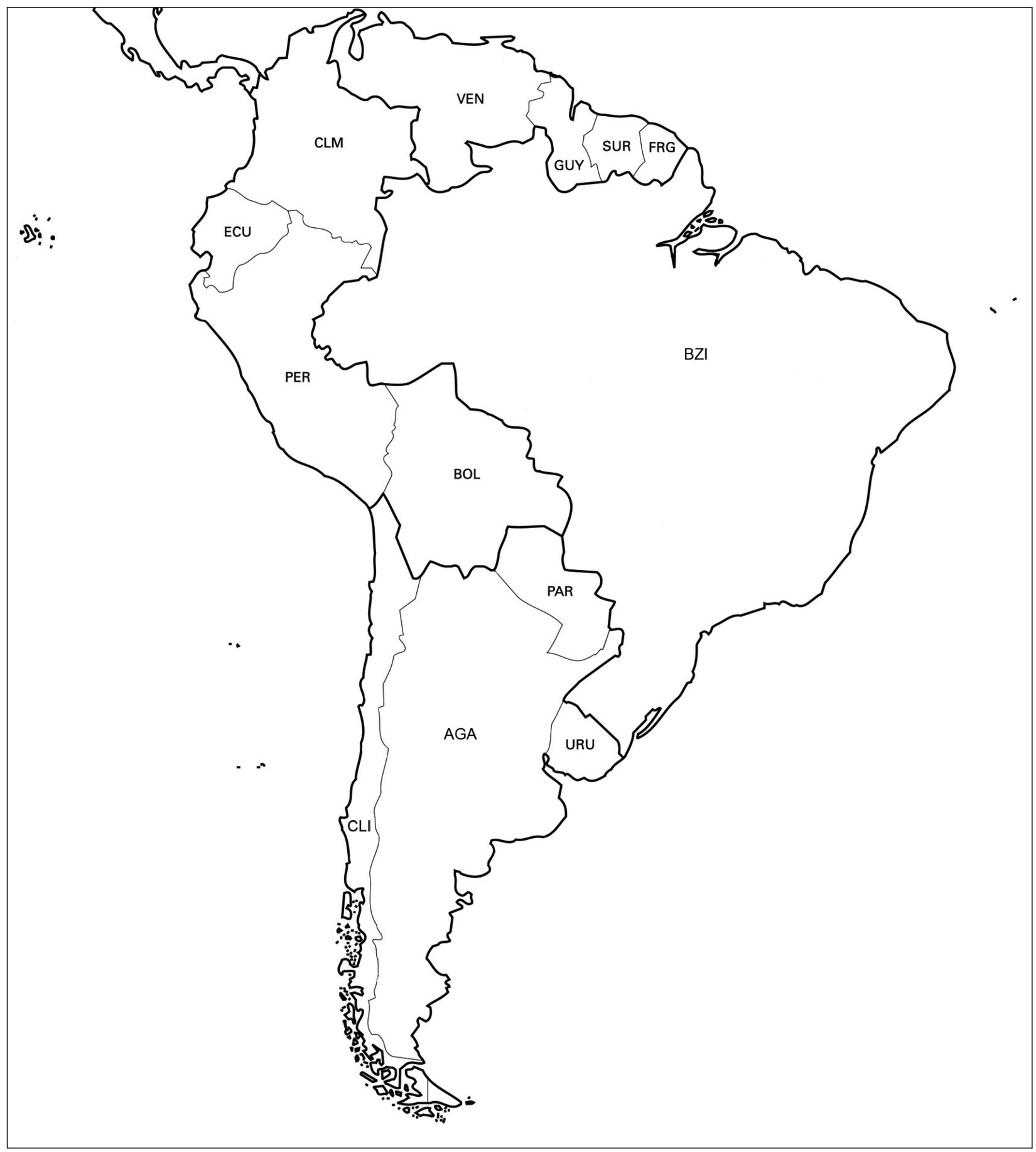

Fig. 2. South America.

\section{South America}

CLM Colombia (Colombian Caribbean Islands and Malpelo Island included)

VEN Venezuela [Venezuelan Antilles and Netherlands Antilles (Curaçao and Bonaire), the island of Aruba included]

GUY Guyana

SUR Suriname

FRG French Guiana
ECU Ecuador (Galápagos Islands included)

PER Peru

BOL Bolivia

BZI Brazil (Fernando de Noronha and Trindade Island included)

PAR Paraguay

URU Uruguay

AGA Argentina (Tierra del Fuego and other islands included)

CLI Chile (Desventurados Islands, Juan Fernández Islands, Tierra del Fuego and other islands included) 


\section{Other criteria followed}

To present the data, the same basic format employed by Lado (1994) in the checklist of Myxomycetes of the Mediterranean countries, has been followed. The review is similar to a checklist, basically a species listing (Table 1), including synonyms, of myxomycetes from the Neotropics cited in the literature, with species distribution by countries. With regard to nomenclature used, the generic and species treatment are those accepted by Lado (2001, 2008) and Hernández-Crespo \& Lado (2005), the generic names Amaurochaete, Ceratiomyxa (traditionally considered a myxomycete until recently) and Hemitrichia are conserved according to Lado \& al. (2005) (see also, Gams, 2005). Infraspecific taxa have not been considered, due to the inconsistency of distinctive characters in many of the cases, and are treated under their respective species. All the species names utilised in the consulted sources have been verified. Spelling variations and transcriptional errors have been corrected. The accepted names appear in bold, and the synonyms (homo or heterotypic synonyms), which have been used for species in the literature from the Neotropics, appear in italics. Records cited herein are compiled from the literature and no attempt has been made, for this paper, to examine or authenticate material.

The taxa, in the Table 1, are arranged alphabetically by genera and species. A query after a species under a certain country, means doubts as to its presence in that country, which is usually due to imprecision by the author of the paper, or the collector of the specimen. The countries or geographical units have been arranged more-or-less in order from the North to the South, in three major bioregions, starting with Central America, continuing with the Caribbean Islands and ending with South America. The totals for the number of species by countries and countries for each species have been given at the end and the side of the table. Doubtful excluded or species have been listed in a separate table (Table 2).

\section{Sources of information}

The monograph by Farr (1976) for the series Flora Neotropica, has been taken as a baseline for this review, and the reference has been included in all the countries for which she gave citations. This may lead to some duplication in the numbers of references for a country, but this has not caused duplication in the records of Myxomycetes, which have been listed only once. All the literature references of Farr's monograph have been incorporated into the list of references below, except those general works that re- compile records or references, such as Lister (1911, 1925), Hagelstein (1944) or Macbride $(1899,1922)$. Some pre-1975 papers, omitted by Farr (1976), are also included. In addition, checklists, inventories, catalogues as well as more obscure papers with valuable information, published after 1975, have been perused for myxomycete records, and used as sources of information for this review. All the sources of information used for the myxomycete records listed in Table 1 are given below, arranged by countries and date. Compilation of this data by country should assist future researchers, and be useful as a guideline for future government initiatives. The sources are:

Argentina (AGA): Spegazzini (1880a, 1880b, 1880c, 1881, 1882, 1886, 1887b, 1889, 1896a, 1896b, 1899a, 1899b, 1909a, 1909b, 1912, 1913, 1919a, 1926, 1927), Berlese (1888), Massee (1889), Saccardo (1892), Saccardo \& Sydow (1899, 1902), Fries (1903), Torrend (1908), Saccardo \& Trotter (1913), Sturgis (1916), Digilio (1946, 1950), Farr (1971, 1973, 1974, 1976), Arambarri (1972, 1973, 1975), Deschamps (1972, 1974, 1975, 1976a, 1976b), Castillo \& al. (1996), Crespo \& Lugo (2003), Wrigley de Basanta \& Stephenson (2005), Wright \& Albertó (2006).

Bahamas (BAH): Britton \& Millspaugh (1920).

Belize (BLZ): Ing \& Haynes (1999).

Bolivia (BOL): Fries (1903), Saccardo \& Saccardo (1906), Torrend (1908), Stevenson \& Cardenas (1949), Farr (1976).

Brazil (BZI): Montagne (1837), Berkeley \& Cooke (1876), Spegazzini (1881, 1888, 1889, 1919b, 1926), Berlese (1888), Massee (1889), Saccardo (1892, 1895), Hennings (1896, 1902a), Bresadola (1896), Pazschke (1896), Saccardo \& Sydow (1899), Jahn (1902, 1904), Saccardo \& Saccardo (1906), Höhnel (1907), Sydow \& Sydow (1907), Torrend (1908, 1915, 1916), Batista (1949), Hashimoto (1953), Hertel (1954a, 1954b, 1955), Farr \& Martin (1958), Farr (1959, 1960, 1968, 1973, 1974, 1976, 1985), Fidalgo \& al. (1965), Ing (1967), Gottsberger (1968, 1971), Mariz (1968), Cavalcanti (1970, 1974a, 1974b, 1976, 1977, 1985, 1996a, 1996b, 2002), Dennis (1970), Gottsberger \& Nannenga-Bremekamp (1971), Mariz \& Cavalcanti (1970), Maimoni-Rodella \& Gottsberger (1980), Bononi \& al. (1981), Cavalcanti \& al. (1982, 1985, 1993, 1999, 2005, 2006), Pôrto \& Cavalcanti (1984, 1986), Pôrto \& al. (1982), Cavalcanti \& Araújo (1985), Cavalcanti \& Dias Filha (1985), Cavalcanti \& Marinho (1985), Cavalcanti \& Oliveira (1985), Cavalcanti \& Porto (1985), Cavalcanti \& Silva (1985), Rodrigues (1985), Santos \& al. (1986), Muchovej \& Muchovej (1987), Capelari \& Mazeiro (1988), Silva \& Cavalcanti (1988), Santos \& Cavalcanti (1988, 1991a, 1991b, 1995), Hochgesand \& Gottsberger (1989, 1996), Hochgesand \& al. (1989), Cavalcanti \& Brito (1990), Mendes \& Guerrero (1990), Rodrigues \& Guerreiro (1990), Rogerson \& al. (1990), Cavalcanti \& Santos (1991), Gottsberger \& al. (1992), Cavalcanti \& Fortes (1994, 1995), Góes Neto (1996), Barbosa (1996), Putzke (1996, 2002), Alves \& Cavalcanti (1996), Cavalcanti \& Putzke (1998), Mobin \& Cavalcanti (1998, 1999a, 1999b, 2000, 2001), Yamamoto \& al. (2000), Cavalcanti \& Mobin (2001, 2002, 2004), Góes Neto \& Cavalcanti (2002), Maimoni-Rodella (2002), Matsumoto (2002), Chiappeta \& al. (2003), Ponte \& al. (2003), Maimoni-Rodella \& Cavalcanti (2006), Bezerra \& al. (2007), Rufino \& Cavalcanti (2007).

Chile (CLI): Bertero (1828), Montagne (1837, 1852a, 1852b), 
Berlese (1888), Massee (1889), Saccardo (1892), Johov (1896), Saccardo \& Sydow (1899), Torrend (1908), Sturgis (1916), Spegazzini (1887b, 1917, 1921), Fries (1920), Mújica \& Vergara (1945), Lazo (1966), Farr (1976), Lado \& al. (2007a).

Colombia (CLM): Leveille (1863), Massee (1889), Saccardo (1892), Torrend (1908), Chardon (1928), Muenscher (1930), Martin (1932, 1938a), Dennis (1970), Farr (1976), Guzmán \& Varela (1978), Uribe-Meléndez (1995).

Costa Rica (COS): Hennings (1902b), Spegazzini (1919b), Welden (1954), Alexopoulos (1967), Alexopoulos \& Sáenz (1975), Farr (1976), Edmunds \& Stephenson (1996), Schnittler \& Stephenson (2000, 2002a, 2002b), Schnittler (2001), Moore \& Stephenson (2003), Stephenson \& al. (2004b), Wrigley de Basanta \& Lado (2005), Rojas \& Stephenson $(2007,2008)$.

Cuba (CUB): Montagne (1837, 1838), Berkeley (1868), Berlese (1888), Massee (1889), Torrend (1908), Saccardo \& Trotter (1913), Farr (1976), Camino (1991, 1996, 1998a, 1998b), Camino \& Pérez (2000, 2001), Pérez \& Camino (2000), Minter \& al. (2001), Camino \& Eliasson (2002), Camino \& Moreno (2002), Camino \& Rodríguez (2002), Krivomaz (2003), Wrigley de Basanta \& al. (2003), Camino \& al. (2005, 2007, in press), Wrigley de Basanta \& Lado (2005).

Dominican Republic (DOM): Ciferri \& González-Fragoso (1926), Toro (1926), Gonzalez-Fragoso \& Ciferri (1927, 1928), Ciferri (1929, 1961), Farr (1976), Minter \& al. (2001),

Ecuador (ECU): Patouillard \& Lagerheim (1891, 1892, 1893 , 1895a, 1985b), Saccardo (1895), Saccardo \& Sydow (1899), Torrend (1908), Bonar (1939), Martin (1948), Harling (1967), Dennis (1970), Eliasson (1971, 2000), Farr (1974, 1976), Farr \& al. (1979), Reid \& al. (1981), Schinner (1981), Eliasson \& Nannenga-Bremekamp (1983), Nannenga-Bremekamp (1989), Stephenson \& Mitchell (1994), Schnittler (2001), Estrada \& al. (2002c), Lado \& al. (2002b), Schnittler \& al. (2002), Schnittler \& Stephenson (2002a, 2002b), Stephenson \& al. (2004a, 2004b), McHugh (2005).

French Guiana (FRG): Farr (1976), Courtecuisse \& al. (1996).

Guatemala (GUA): Farr (1976), Estrada-Torres \& al. (2000).

Guyana (GUY): Montagne (1855), Cooke (1877), Berlese (1888), Torrend (1908), Gilbert (1928), Farr (1976), Rogerson \& al. (1990).

Haiti (HAI): Benjamín \& Slott (1969), Farr (1976), Minter \& al. (2001).

Honduras (HON): Davis \& Butterfield (1967), Alexopoulos (1970), Clark \& Collins (1973), Farr (1976).

Jamaica (JAM): Saccardo (1895), Torrend (1908), Alexopoulos \& Beneke (1954a, 1954b), Farr (1957, 1974, 1976), Alexopoulos (1967, 1970), Minter \& al. (2001).

Leeward Islands (LEE): Lister (1898a, 1898b), Saccardo \& Sydow (1902), Duss (1903, 1904), Seaver \& Chardon (1926), Raunkiaer (1928), Hagelstein (1932), Alexopoulos (1970), Stevenson (1975), Farr (1976), Minter \& al. (2001).

Mexico (MEX): Saccardo \& Sydow (1902), Torrend (1908), Emoto (1933), Welden \& Lemke (1961), Alexopoulos \& Blackwell (1968), Martínez-Murillo \& Ochotorena (1970), Guzmán (1972, 1983), Farr (1976), Braun \& Keller (1976, 1986), Keller \& Brooks (1976), Keller \& Braun (1977), Welden \& Guzmán (1978), Welden \& al. (1979), Guzmán \& Varela (1979), López \& al. (1979, 1981a, 1981b, 1981c, 1982), Pérez-Silva (1979), GómezSánchez \& Castillo (1981), Dávalos \& Guzmán (1981), Guzmán \& Guzmán-Dávalos (1981), Mapes \& al. (1981), López \& Sosa (1982), Martínez-Alfaro \& al. (1983), Villarreal (1983, 1985, 1990), Chacón \& Guzmán (1984), Gómez-Pompa \& al. (1984),
Guzmán \& Villarreal (1984), Pérez-Silva \& Aguirre-Acosta (1985), Trujillo-Flores \& al. (1986), Trujillo-Flores (1988), PérezMoreno \& Villarreal (1988), Heredia (1989), Capello-García \& Hernández-Trejo (1990), Hernández-Cuevas \& al. (1991), Galindo-Flores (1992), Galindo-Flores \& Estrada-Torres (1993), Galindo-Flores \& al. (1993), Ogata \& al. (1994, 1996), EstradaTorres (1996), Illana (1996), Lizárraga \& al. (1996, 1997, 1998, 1999a, 1999b, 1999c, 2003 a, 2003b, 2004a, 2004b, 2005a, 2005b, 2006, 2007, 2008), López \& García (1996a, 1996b, 1996c, 1996d, 1996e, 1996f, 1996g, 1996h, 1996i, 1996j, 2001a, 2001b, 2001c, 2001d, 2002a, 2002b, 2002c, 2002d, 2002e, 2002f, 2002g, 2005a, 2005b), Ogata \& Andrade-Torres (1996), Rodríguez-Palma \& Estrada-Torres (1996a, 1996b), Hernández-Cuevas \& EstradaTorres (1993a, 1993b, 1997), Rodríguez-Palma \& al. (1996, 2002, 2005), Moreno \& al. (1997a, 1997b, 1997c, 2000, 2001, 2004, 2006a, 2006b, 2007), Andrade-Torres (1998), Rodríguez-Palma (1998), Illana \& al. (1999, 2000), Lado \& al. (1999a, 1999b, 2002a, 2003, 2007b), Pérez-Silva \& Bárcenas (1999), Mosquera \& al. (2000a, 2000b, 2003), Yamamoto (2000), Estrada-Torres \& al. (2001, 2002a, 2002b, 2002c, 2002d, 2003, 2005, in press), PérezSilva \& al. (2001), Andrade-Torres \& al. (2002a, 2002b), Lizárraga (2002), Stephenson \& al. (2003, 2004a, 2004b), Wrigley de Basanta \& al. (2002, 2003, 2008, in press), Wrigley de Basanta \& Lado (2005).

Nicaragua (NIC): Macbride (1893), Saccardo (1895), Macbride \& Smith (1896), Torrend (1908), Farr (1976).

Panama (PAN): Standley (1927, 1933), Weston (1933), Martin (1936, 1938b, 1957), Welden (1954), Davis \& Butterfield (1967), Dennis (1970), Ling \& Collins (1970), Farr (1974, 1976), Wheeler (1980), Pando (1997).

Paraguay (PAR): Hennings (1896), Saccardo \& Trotter (1913), Spegazzini (1886, 1888, 1919b, 1923, 1926), Farr (1973, 1976).

Peru (PER): Rudolphi (1829), Farr (1976), Stephenson \& Mitchell (1994), Wrigley de Basanta \& Lado (2005), Wrigley de Basanta \& al. (2008).

Puerto Rico (PUE): Klotzsch (1852), Seaver \& Chardon (1926), Hagelstein (1927, 1932), Alexopoulos (1970), Stevenson (1975), Farr (1976), Stephenson \& al. (1999, 2004b), Novozhilov \& al. (2000), Minter \& al. (2001), Schnittler (2001), Nieves-Rivera \& Darrah (2002a, 2002b), Schnittler \& Stephenson (2002a, 2002b), Nieves-Rivera (2003), Wrigley de Basanta \& Lado (2005), Wrigley de Basanta \& al. (2008).

Surinam (SUR): Gilbert (1928), Nannenga-Bremekamp (1961), Farr (1976), Rogerson \& al. (1990).

Trinidad and Tobago (TRT): Rorer (1911), Baker \& Dale (1951), Barnes (1963), Alexopoulos (1970), Dennis (1970), Farr (1976), Minter \& al. (2001).

Uruguay (URU): Spegazzini $(1881,1926)$, Herter $(1907,1933$, 1939), Farr (1976), García-Zorrón $(1967,1977)$.

Venezuela (VEN): Berlese (1888), Patouillard \& Gaillard (1888), Massee (1889), Saccardo (1892, 1895), Torrend (1908), Saccardo \& Trotter (1913), Heim (1928), Muenscher (1934), Rodríguez (1955, 1957), Dennis (1960, 1970), Farr \& Kowalski (1974), Farr (1974, 1976), Buyck (1984), Verde de Millán \& Jaimes (1987), Rogerson \& al. (1990).

Windward Islands (WIN): Cooke (1889), Lister (1898a), Duss (1903, 1904), Chardon (1926), Hagelstein (1932), Alexopoulos (1967, 1970), Farr (1969, 1976), Dennis (1970), Minter \& al. (2001). 


\begin{tabular}{|c|c|c|c|c|c|c|c|c|c|c|c|c|c|c|c|c|c|}
\hline |e701 & & $\sim$ & in & $\stackrel{\infty}{\sim}$ & $N$ & $\stackrel{2}{2}$ & in & - & - & 0 & $\div$ & $\div$ & 0 & $\sim$ & in & - & $\simeq$ \\
\hline еипุuəб. & 委 & & & 委 & 芯 & $\begin{array}{l}\text { 采 } \\
\end{array}$ & 帒 & 帒 & 要 & & 正 & 㯊 & & & $\frac{\pi}{3}$ & & 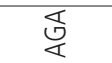 \\
\hline ә!!૫ગ & $\bar{U}$ & & & $\bar{U}$ & & $\bar{\Xi}$ & & & & & 己 & 己 & & & & & \\
\hline Kenbnun & 록 & & & 옥 & & 옥 & 록 & & & & & 롱 & & & & & \\
\hline Kenbesed & 愛 & & & & & & $\frac{\alpha}{\alpha}$ & & & & 愛 & & & & & & $\frac{\alpha}{\alpha}$ \\
\hline e!n!।0g & $\vec{\varnothing}$ & & & $\vec{\varnothing}$ & & $\vec{\varnothing}$ & & & & & & $\vec{\varnothing}$ & & & & & \\
\hline nגəd & 㗂 & & & 㗂 & & 临 & & & & & & & & & & & \\
\hline sopenj] & 己. & 己己 & 己. & 己. & & 己. & 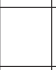 & & & 己. & 己. & $\sim$ & & 己. & & & 己己 \\
\hline |lzedg & $\bar{\infty}$ & & & $\bar{\infty}$ & $\bar{\aleph}$ & $\bar{\infty}$ & $\bar{\infty}$ & & & $\bar{\infty}$ & $\bar{\infty}$ & $\bar{\infty}$ & $\bar{\infty}$ & $\bar{\infty}$ & $\bar{\infty}$ & & $\bar{\infty}$ \\
\hline eue!ng чวนә. & ষ্ৰ & & & 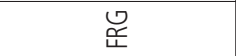 & & ষ্ৰে & & & & & & 荘 & & & & & \\
\hline әmeuluns & $\stackrel{\circ}{\sim}$ & & & $\stackrel{\text { 兴 }}{\curvearrowleft}$ & & $\stackrel{o}{\sim}$ & & & & & & & & & & & \\
\hline euerng & डे & & & ड़े & & डे & & & & & & & & & & & \\
\hline ерәпzәиә^ & 妾 & & & z & & 政 & & & & & 妾 & z & & & & & $\overline{\mathrm{z}}$ \\
\hline elquo|0J & $\sum$ & & & $\sum$ & & $\sum$ & & & & $\sum$ & $\sum$ & $\sum$ & & & & & \\
\hline oбeqoı pue pep!u!. 1 & 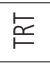 & & & 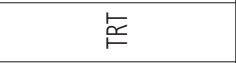 & & $\underline{\underline{\mu}}$ & & & & & 点 & $\stackrel{F}{\underline{F}}$ & & & & & 点 \\
\hline spue|ss рлемриім. & $\geqq$ & & & 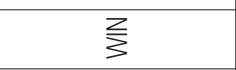 & & 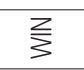 & & & & & 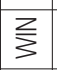 & & ऐ & & & & \\
\hline spue|sı рıемәә7 & 岃 & & & 岃 & & 岃 & & & & & 出 & 出 & & & & & 岃 \\
\hline ox!y oflənd & 容 & & 容 & 容 & & 㟔 & & & & 容 & 容 & 容 & & & & & \\
\hline еиез!uाuog 'dəy & 음 & & & ¿ & & ò & & & & & & & & & & & \\
\hline !!?еH & 竎 & & & 竎 & & & & & & & 竎 & & & & & & \\
\hline eșemer & $\underset{1}{i}$ & & & $\sum_{1}$ & & $\underset{1}{\$}$ & & & & & 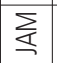 & 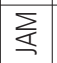 & & & & & $\underset{1}{\$}$ \\
\hline eqno & 弅 & & 孚 & 弅 & & 弅 & & & & 弅 & 弅 & $\stackrel{9}{\mathrm{O}}$ & 敛 & & & & 弅 \\
\hline semeyeg & 甶 & & & 壳 & & 䓌 & & & & & & & & & & & \\
\hline emeued & 斑 & & & 交 & & 交 & & & & & & 斑 & 交 & & z & & 斑 \\
\hline 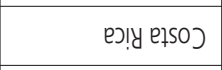 & $\tilde{\ominus}$ & & $\tilde{U}$ & $\tilde{\mathrm{U}}$ & & $\tilde{\vartheta}$ & & & & & $\tilde{\mathrm{U}}$ & $\tilde{\mathrm{U}}$ & $\tilde{\mathrm{o}}$ & & $\tilde{\mathrm{U}}$ & & $\tilde{\mathrm{U}}$ \\
\hline enбe.⿲丿尸!N & $\frac{u}{z}$ & & & $\frac{u}{z}$ & & $\frac{u}{z}$ & & & & & & & & & & & \\
\hline Lopenes I & บ & & & & & & & & & & & & & & & & \\
\hline sempuoH & 호 & & & 호 & & 호 & & & & & & & & & & & \\
\hline ерешәдеn & 竎 & & & 今 & & & & & & & & & & & & & \\
\hline әz:|әд & 금 & & & 금 & & 금 & & & & & & & & & & & \\
\hline $0 כ \times x \partial W$ & 盖 & 售 & 离 & 离 & & 蛍 & 售 & & & 崔 & 崔 & 垐 & 离 & & 㠍 & 嵫 & 紋 \\
\hline & & 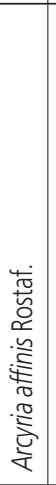 & 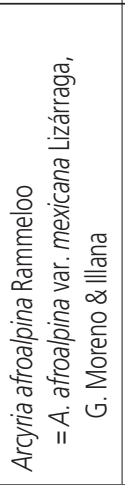 & 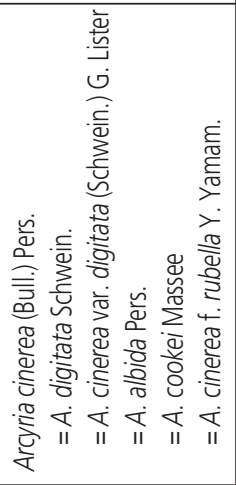 & 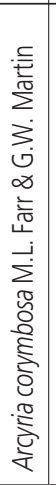 & 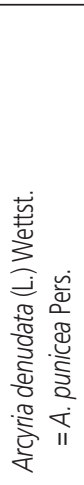 & 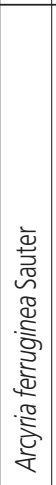 & 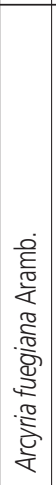 & 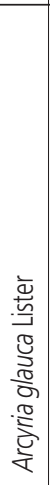 & 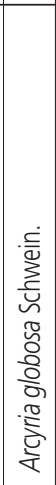 & 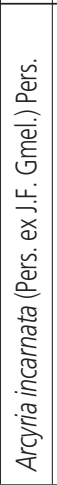 & 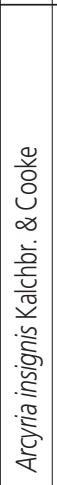 & 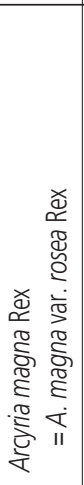 & 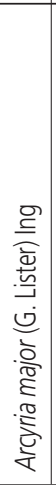 & 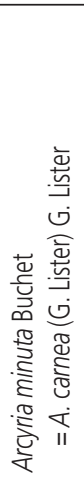 & 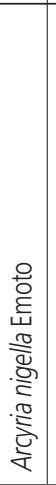 & 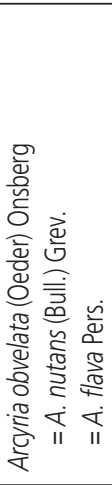 \\
\hline
\end{tabular}




\begin{tabular}{|c|c|c|c|c|c|c|c|c|c|c|c|c|c|c|c|c|c|c|c|c|}
\hline n & a & Ln & - & $\sigma$ & - & $\sigma$ & $m$ & - & $N$ & - & - & a & a & $\sigma$ & $\infty$ & $N$ & - & $\sim$ & $N$ & $\sim$ \\
\hline \multirow[t]{3}{*}{ 正 } & 褰 & & & 过 & & 正 & 预 & & 昰 & & & 玻 & 哥 & & 昏 & & & & 过 & \\
\hline & & & & $\bar{U}$ & & こ & & $\bar{U}$ & & & & $\bar{u}$ & $\bar{U}$ & & 己 & & $\bar{U}$ & & & \\
\hline & & & & & & & & & & & & 록 & & & 록 & & & & & \\
\hline \multirow[t]{4}{*}{ 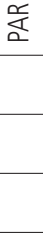 } & & & & & & & & & & & & & & & & & & & & \\
\hline & & & & & & & & & & & & $\vec{D}$ & & 。्ठ & ठ & & & $\overrightarrow{\mathrm{o}}$ & & \\
\hline & & & & & & & & & & & & & & & & & & & & \\
\hline & 己્u & & & 己્ّ & & & & & & 己્ & & & 己己 & & 己. & & & & & \\
\hline $\bar{\infty}$ & $\bar{\infty}$ & $\bar{\infty}$ & $\bar{\infty}$ & $\bar{\infty}$ & $\bar{\infty}$ & & & & & & & $\bar{\infty}$ & $\bar{\infty}$ & $\bar{\infty}$ & $\bar{\aleph}$ & $\bar{\infty}$ & & & & $\bar{\infty}$ \\
\hline & & & & & & & & & & & & & & & & & & & & \\
\hline & & & & & & & & & & & & & & & & & & & & \\
\hline & & & & & & & & & & & & & & & & & & & & \\
\hline 妾 & 妾 & & & & & & & & & & & z & & & & & & & & \\
\hline & $\sum_{\mathcal{U}}$ & $\sum$ & & & & $\sum$ & $\sum$ & & & & & & & & & & & & & \\
\hline & & & & & & & & & & & & & & & & & & & & \\
\hline & & & & 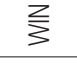 & & & & & & & & & & & 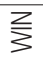 & & & & & $\sim$ \\
\hline & & & & 出 & & & & & & & & 出 & 岃 & & & & & & & \\
\hline & 㟔 & & & & & & & & & & & & 嵓 & & & & & & & \\
\hline & & ¿ & & & & & & & & & & & & ¿ & & & & & & \\
\hline & & & & & & & & & & & & & & & & & & & & \\
\hline & $\sum_{\leq}$ & $\gtreqless$ & & $\sum_{\leq}$ & & & & & & & & & & & & & & & & \\
\hline $\mathrm{g}$ & & & & & & & & & & & & & $\mathrm{g}$ & & & & & & & \\
\hline$\sim$ & & & & & & & & & & & & & & & & & & & & \\
\hline$z_{a}$ & 充 & & & 充 & & & & & & & & & $z_{a}$ & & & & & & & \\
\hline$\sim$. & & & & & & & $\tilde{\mathcal{O}}$ & & & & & $\sim$ & & & & & & & & \\
\hline & & & & & & & & & & & & & & & & & & & & \\
\hline & & & & & & & & & & & & & & & & & & & & \\
\hline & & & & & & & & & & & & & & & & & & & & \\
\hline & & & & & & & & & & & & 今 & & & & & & & & \\
\hline & & & & & & & & & & & & & & & & & & & & \\
\hline 岀 & 坟 & 离 & & 离 & & 离 & & & 离 & & 离 & 售 & 离 & 崖 & 离 & 觉 & & 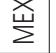 & 紋 & 离 \\
\hline 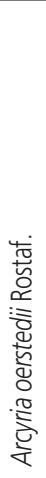 & 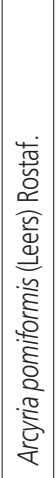 & 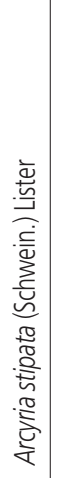 & 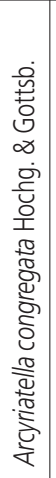 & 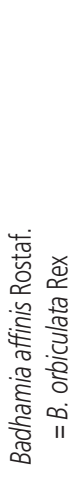 & 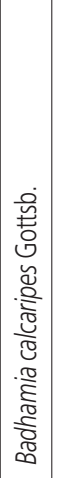 & 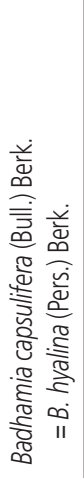 & 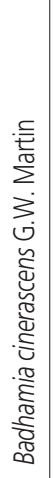 & 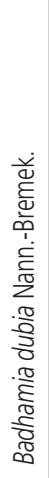 & 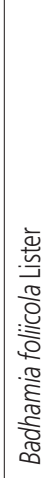 & 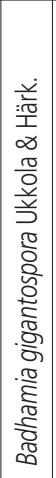 & 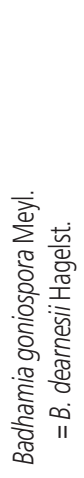 & 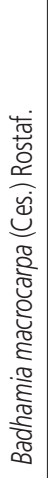 & 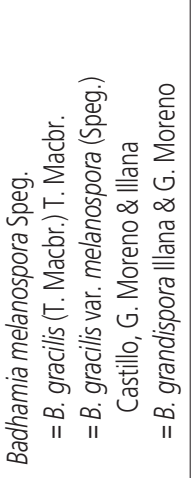 & 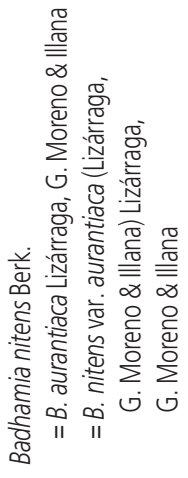 & 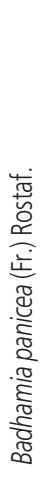 & 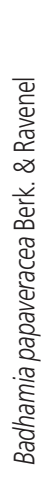 & 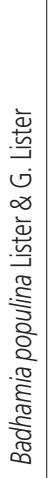 & 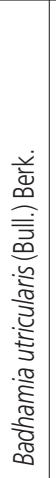 & 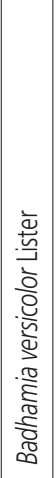 & 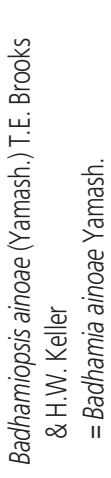 \\
\hline
\end{tabular}




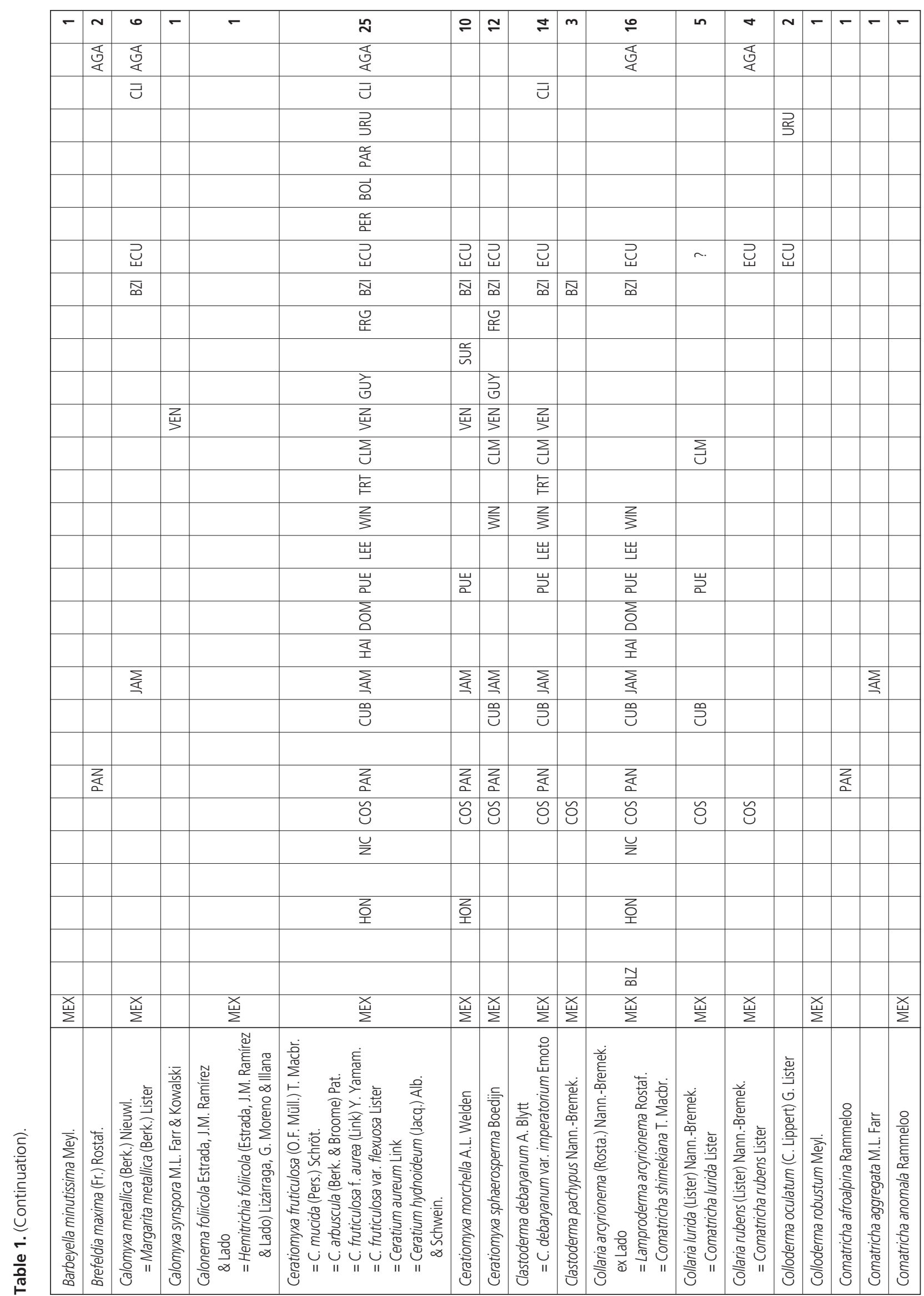




\begin{tabular}{|c|c|c|c|c|c|c|c|c|c|c|c|c|c|c|c|c|c|c|}
\hline- & $\Phi$ & - & $=$ & - & - & $\simeq$ & 으 & - & $\simeq$ & $\simeq$ & in & $=$ & $\sigma$ & $m$ & $\sigma$ & $\nabla$ & - & $n$ \\
\hline 要 & $\begin{array}{l}\mathbb{3} \\
\text { d }\end{array}$ & & 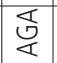 & & & 委 & 巢 & & 昏 & 芯 & & 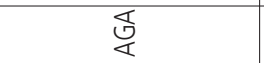 & & 委 & 委 & 要 & & 要 \\
\hline & 己 & & $\bar{u}$ & & & $\bar{J}$ & & & & & & & & & & & & $\bar{v}$ \\
\hline & & & & & & & 록 & & & & & & & $\overrightarrow{\text { Pे }}$ & & & & \\
\hline & & & & & & & & & & & & & & & $\frac{\alpha}{\alpha}$ & & & \\
\hline & & & & & & $\vec{\triangleright}$ & $\overrightarrow{\mathrm{D}}$ & & & & & $\vec{\varnothing}$ & & & & & & \\
\hline & & & & & & & & & 㽞 & 吕 & & & & & & & & \\
\hline & 己. & & & & & 己 & 己己 & & 己己 & 己己 & 己己 & 己 & & & 己 & & & \\
\hline & $\bar{\aleph}$ & & $\bar{\infty}$ & & $\bar{\infty}$ & 怘 & $\bar{\aleph}$ & & $\bar{\infty}$ & $\bar{\infty}$ & & $\bar{\aleph}$ & $\bar{\aleph}$ & & న్థ & $\bar{\aleph}$ & & $\bar{\infty}$ \\
\hline & & & & & & শ্্̛山 & & & & & & $\underset{\Psi}{\Psi}$ & & & ঙ্ষ4 & & & \\
\hline & & & & & & & & & & & & & & & & & & \\
\hline & & & & & & & & & & & & & & & & & & \\
\hline & z & & 妾 & & & & 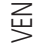 & & 妾 & $\vec{z}$ & & 水 & & & 忌 & & & 妾 \\
\hline & 仙 & & & & & & & & & $\sum$ & $\sum$ & 住 & $\sum_{\mathcal{U}}$ & & $\sum_{\mathcal{U}}$ & & & \\
\hline & 店 & & & & & & & & & & & 扂 & & & & & & \\
\hline & 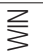 & & & & & & & & & $\frac{2}{3}$ & & $z_{3}$ & & & & & & \\
\hline & & & 出 & & & 岁 & & & 岃 & & & 岃 & & & & & & \\
\hline & 嵓 & & 容 & & & & 嵓 & & 容 & 岂 & & 容 & & & & & & \\
\hline & & & & & & & & & & $\overline{8}$ & & & & & & & & \\
\hline & 至 & & & & & & & & & & & & & & & & & \\
\hline & $\sum$ & & & & & $\sum$ & & & 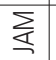 & $\sum$ & $\sum$ & $\sum$ & & & & & & $\sum_{\leq}$ \\
\hline & $\stackrel{m}{\mathrm{Z}}$ & & 学 & & & ב⿱山己 & & & 学 & & בे & $\stackrel{m}{\mathrm{~J}}$ & 忿 & & & & & \\
\hline & & & & & & & & & & & & & & & & & & \\
\hline & & & 㝊 & & & za & za & & & & & zax & & & za & $z_{a}$ & & 亲 \\
\hline & ֻัن & & $\widetilde{\mathcal{O}}$ & & & $\tilde{O}$ & $\tilde{O}$ & & $\tilde{U}$ & $\tilde{O}$ & $\tilde{O}$ & $\tilde{O}$ & & & & & & \\
\hline & & & & & & & & & & & & & & & & & & \\
\hline & & & & & & & & & & & & & & & & & & \\
\hline & & & & & & & & & & & & & & & & & & \\
\hline & & & $\begin{array}{l}3 \\
\end{array}$ & & & & & & & & & 今 & & & & & & \\
\hline & & & & & & & & & 그 & & & & & & & & & \\
\hline & 崖 & 前 & 﨎 & 离 & & 崖 & $\stackrel{\text { 离 }}{\Sigma}$ & 离 & 离 & 离 & & 崖 & 离 & 宸 & 崖 & 离 & 註 & 觉 \\
\hline 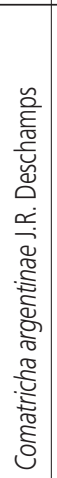 & 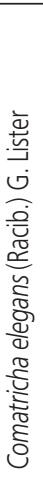 & 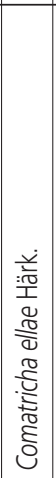 & 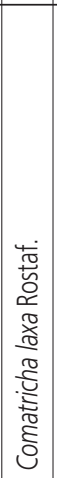 & 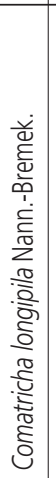 & 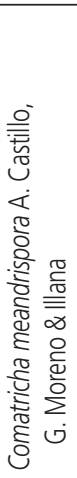 & 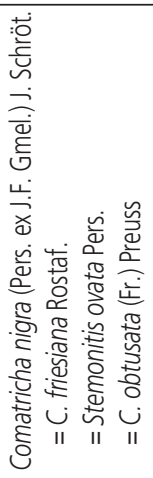 & 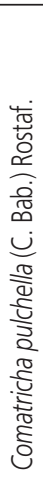 & 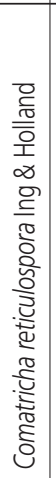 & 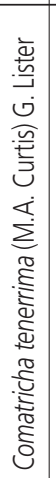 & 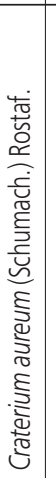 & 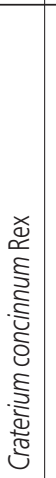 & 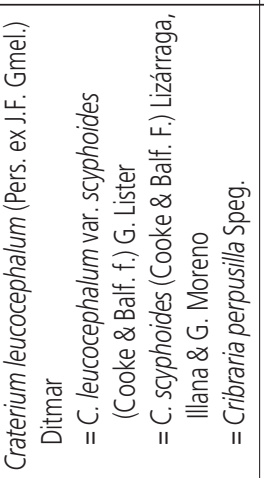 & 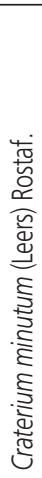 & 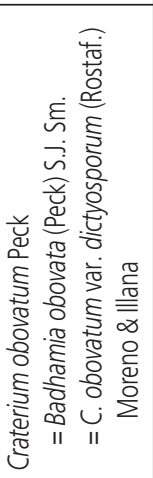 & 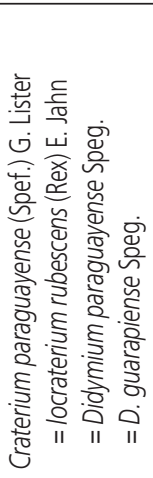 & 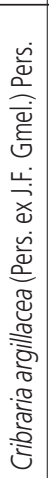 & 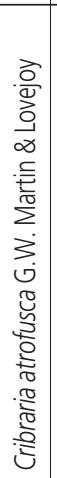 & 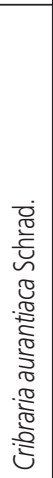 \\
\hline
\end{tabular}




\begin{tabular}{|c|c|c|c|c|c|c|c|c|c|c|c|c|c|c|c|c|c|c|c|c|c|c|c|}
\hline$\tilde{\sim}$ & $\sigma$ & - & $N$ & - & - & \pm & $=$ & - & - & $m$ & - & $\stackrel{\nabla}{ }$ & $\infty$ & $\sigma$ & $\sim$ & 6 & $N$ & - & - & - & $n$ & \pm & $\Xi$ \\
\hline 委 & & & & & & & & & & & & & & & & & & & & 褰 & & $\mathbb{4}$ & 委 \\
\hline $\bar{U}$ & & & & & & & & & & $\bar{U}$ & $\sim$. & & & $\bar{U}$ & & $\bar{u}$ & & & & & $\bar{U}$ & & \\
\hline 록 & & & & & & & & & & & & & $\stackrel{\vec{g}}{\vec{g}}$ & & & & & & & & & & 록 \\
\hline & & & & & & & & & & & & & & & & & & & & & & & \\
\hline$\vec{\varnothing}$ & & & & & & $\overrightarrow{\mathrm{D}}$ & & & & & & & & & & & & & & & & & \\
\hline 獣 & & & & & & & & & & & & & & & & & & & & & & & \\
\hline 己. & 己. & & & & & 己. & 己. & & & & & & & & & & & & & & & 己己 & 검 \\
\hline $\bar{্}$ & $\bar{\infty}$ & & $\bar{\aleph}$ & & & $\bar{\aleph}$ & $\bar{\triangleright}$ & $\bar{\infty}$ & & & & $\bar{\infty}$ & $\bar{\infty}$ & $\bar{\infty}$ & & $\bar{\aleph}$ & & & & & $\bar{\aleph}$ & $\bar{\infty}$ & $\bar{\aleph}$ \\
\hline $\mathscr{\Psi}$ & & \begin{tabular}{|l} 
\\
的 \\
\end{tabular} & & & & & & & & & & 마⿺ & & & & & & & & & & & \\
\hline & & & & & & & & & & & & & & & & & & & & & & & \\
\hline & & & & & & & & & & & & & & & & & & & & & & & \\
\hline 妾 & & & & & & 妾 & 妾 & & & & & 妾 & & & & & 妾 & & & & 妾 & $\mathrm{z}$ & 妾 \\
\hline$\sum$ & & & & & & $\sum$ & & & & $\sum$ & & $\sum$ & & & & & & & & & & $\sum_{U}$ & \\
\hline 哀 & & & & & & 产 & 占 & & & & & $\stackrel{t}{\mathscr{E}}$ & $\underline{F}$ & & & & & & & & & & $\stackrel{\underline{F}}{\underline{I}}$ \\
\hline 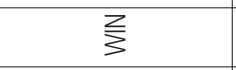 & $\gtreqless$ & & & & & $\gtreqless$ & $\geqq$ & & & & & $z_{3}$ & $\geqq$ & & & & & & & & & $\geqq$ & $\geqq$ \\
\hline 岁 & & & & & & 出 & 出 & & & & & 岃 & 出 & & & & & & & & 出 & 出 & 岃 \\
\hline 岁 & & & & & & 嵓 & 嵓 & & & & & 岁 & & & & & & & & & & & 岁 \\
\hline & & & & & & & & & & & & & & & & & & & & & & & \\
\hline & & & & & & & & & & & & & & & & & & & & & & 㕵 & \\
\hline 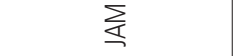 & & & & & & $\sum_{\leq}$ & $\sum_{\leq}$ & & & & & $\sum_{\leq}$ & $\sum_{\leq}$ & & & & & & & & $\sum_{4}$ & $\sum_{i}$ & $\sum_{\leq}$ \\
\hline$\stackrel{9}{己}$ & & & & & & $\stackrel{m}{己}$ & & & & & & $\stackrel{9}{己}$ & & & & & & & & & & $\stackrel{M}{己}$ & $\stackrel{9}{己}$ \\
\hline 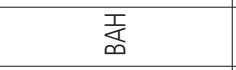 & & & & & & & & & & & & & & & & & & & & & & & \\
\hline$z_{a}$ & & & & & & $z_{\alpha}$ & $z_{\alpha}$ & & & & & ż & & & & $z_{\alpha}$ & & & & & & za & 否 \\
\hline$\tilde{u}$ & & & & & & $\tilde{U}$ & $\tilde{u}$ & & & & & $\tilde{u}$ & $\tilde{u}$ & $\tilde{u}$ & $\tilde{U}$ & $\tilde{u}$ & & & & & $\check{u}$ & $\tilde{u}$ & $\tilde{u}$ \\
\hline$\frac{u}{z}$ & & & & & & & & & & & & & & & & & & & & & & & \\
\hline & & & & & & & & & & & & & & & & & & & & & & & \\
\hline & & & & & & & & & & & & & & & & & & & & & & 오 & \\
\hline S & & & & & & & & & & & & & & & & S & & & & & & & \\
\hline 그 & & & & & & & & & & & & 그 & & & & & & & & & & & \\
\hline 学 & 寽 & & 䖝 & 学 & 学 & 嵒 & 学 & & 盖 & 峃 & 獃 & 离 & 峃 & 曽 & 痥 & 妥 & 蔍 & 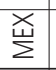 & 前 & & 售 & 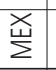 & 离 \\
\hline 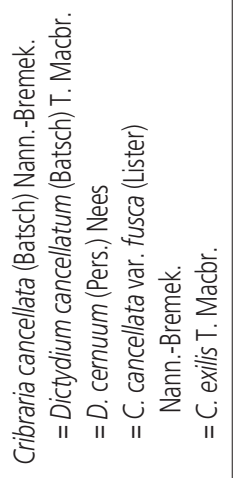 & 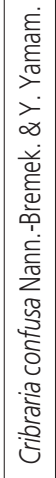 & 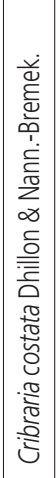 & 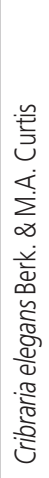 & 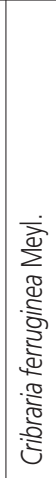 & 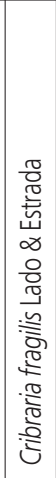 & 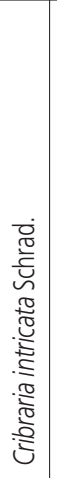 & 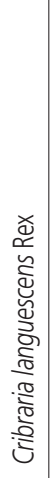 & 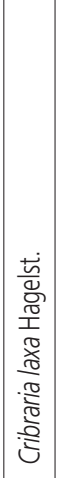 & 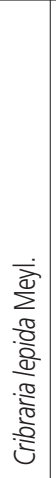 & 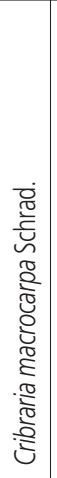 & 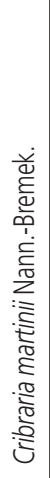 & 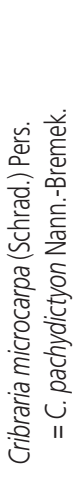 & 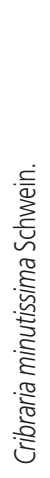 & 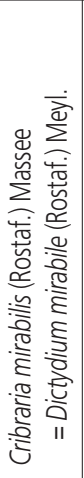 & 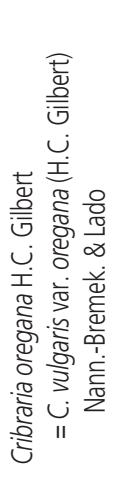 & 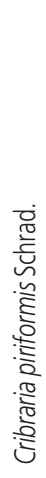 & 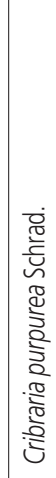 & 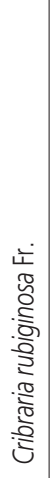 & 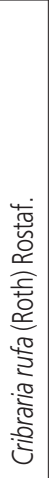 & 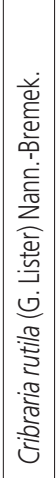 & 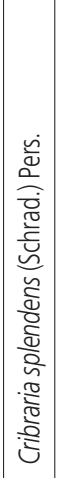 & 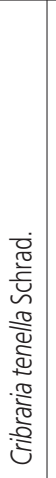 & 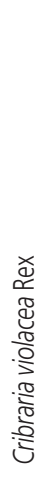 \\
\hline
\end{tabular}




\begin{tabular}{|c|c|c|c|c|c|c|c|c|c|c|c|c|c|c|c|c|c|c|c|c|}
\hline$\sigma$ & - & $=$ & 음 & $m$ & $\sim$ & $m$ & - & - & - & $\simeq$ & - & - & $\sim$ & $\sim$ & $\infty$ & $\sim$ & - & $\nabla$ & - & $m$ \\
\hline $\begin{array}{l}\text { 采 } \\
\end{array}$ & & & 正 & & & & & & & 委 & & 采 & 芯 & 秀 & & & & & & \\
\hline & & & $\bar{J}$ & $\overline{\text { J }}$ & & & & & & $\bar{\nu}$ & & & $\bar{U}$ & & & & & & & \\
\hline & & & 록 & & & & & & & & & & & & & & & & & \\
\hline & & & $\frac{\alpha}{\frac{\alpha}{\alpha}}$ & & & & & & & & & & & & & & & & & \\
\hline & & & $\vec{\circ}$ & & & & & & & & & & & & & & & & & \\
\hline & & & & & & & & & & & & & & & & & & & & \\
\hline & & 겄 & 己. & & & & & & & & & & & & 겁 & 己. & & 己. & & \\
\hline $\bar{\infty}$ & & $\bar{\infty}$ & $\bar{\infty}$ & & & & & & & $\bar{\infty}$ & & & & & $\bar{\infty}$ & & $\bar{\infty}$ & $\bar{\infty}$ & & $\bar{\triangleright}$ \\
\hline
\end{tabular}

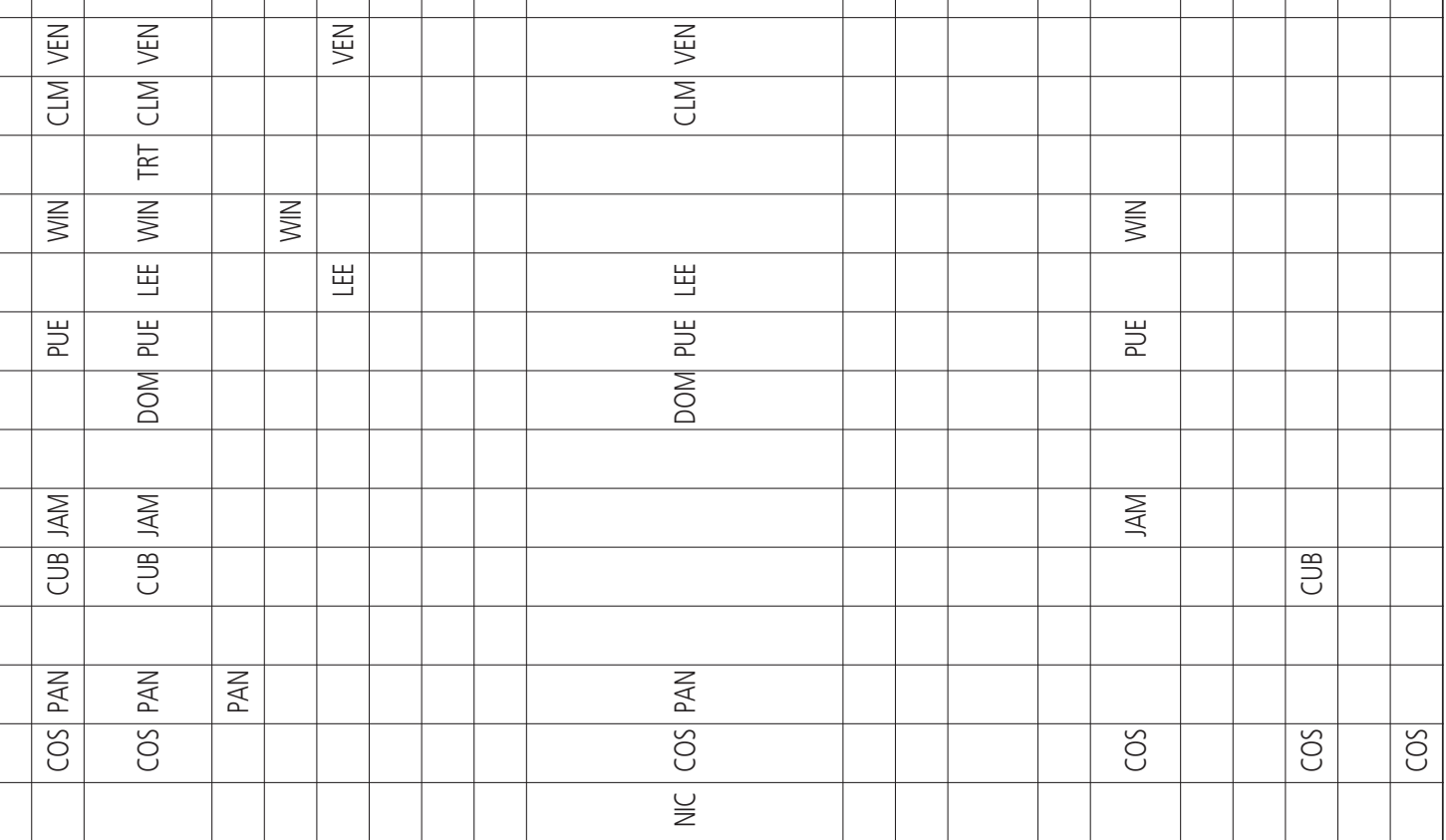

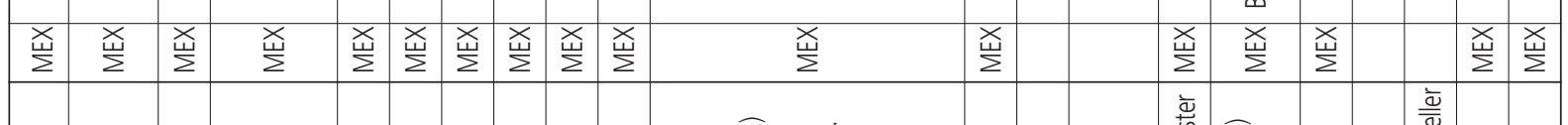

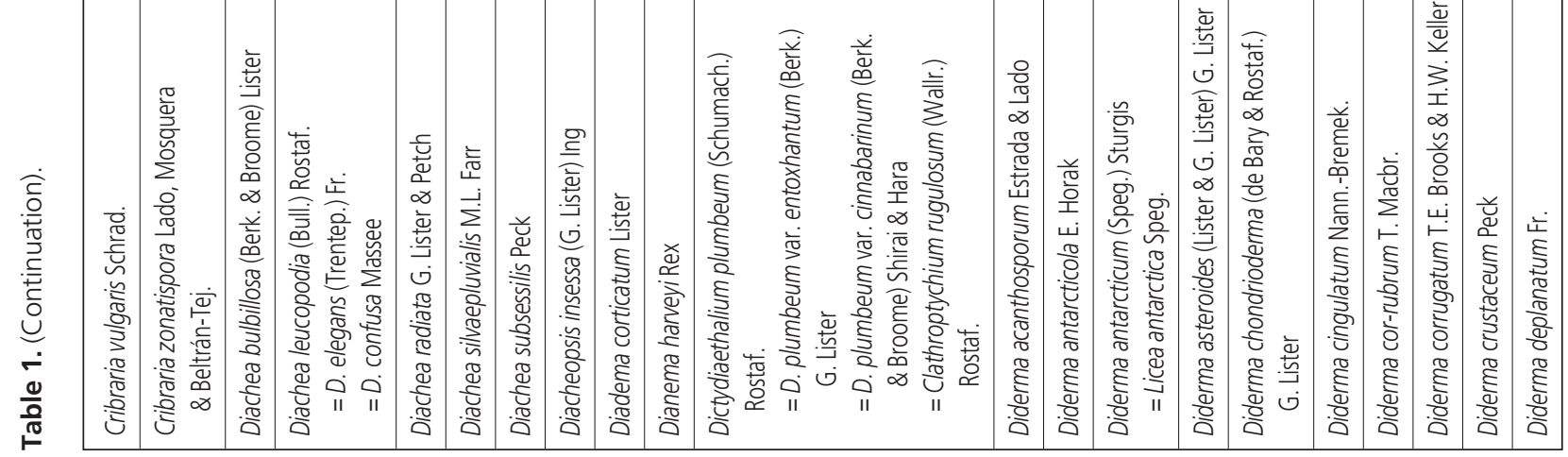




\begin{tabular}{|c|c|c|c|c|c|c|c|c|c|c|c|c|c|c|c|c|c|c|c|c|c|}
\hline 우 & - & - & - & - & $\sigma$ & - & $\stackrel{\infty}{\simeq}$ & - & $\sim$ & - & in & - & $\sigma$ & $N$ & $1-$ & 6 & $N$ & $m$ & - & - & $m$ \\
\hline 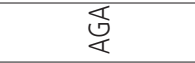 & & & 自 & 芯 & 芯 & 芯 & 过 & & & & $\mathbb{4}$ & & 正 & & 采 & & & & & & 芯 \\
\hline$\sim$ & $\bar{\beth}$ & & & & & & & & & & 己 & & $\bar{\beth}$ & & & & & & & 己 & 己 \\
\hline \multirow[t]{4}{*}{ 곡 } & & & & & & & 롣 & & & & & & & & & & & & & & \\
\hline & & & & & & & & & & & & & & & & & & & & & \\
\hline & & & & & & & & & & & & & & & & & & & & & $\overrightarrow{\mathrm{O}}$ \\
\hline & & & & & 䍃 & & 哭 & & & & & & & & & & & & & & \\
\hline 己. & & & & & 己. & & 己. & & 总 & & & & & 怠 & & & ב. & & 己. & & \\
\hline$\overline{\check{\infty}}$ & & & & & & & $\bar{\aleph}$ & & & & & & & & & & & & & $\sim$ & $\bar{\aleph}$ \\
\hline & & & & & & & & & & & & & & & & & & & & & \\
\hline & & & & & & & & & & & & & & & & & & & & & \\
\hline & & & & & & & & & & & & & & & & & & & & & \\
\hline 妾 & & & & & 妾 & & 妾 & 妾 & & 妾 & & & & & & & & 妾 & & & 妾 \\
\hline$\sum_{U}$ & & & & & & & $\sum$ & & & & $\sum_{\mathcal{U}}$ & & $\sum_{\mathcal{U}}$ & & & & & & & & \\
\hline$\underline{\underline{E}}$ & & & & & & & 占 & & & & & & & & & 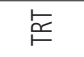 & & & & & \\
\hline$\sum_{3}$ & & & & & & & 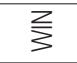 & & & & & & & & & $\sum_{3}$ & & & & & $\frac{z}{3}$ \\
\hline 出 & & $\sim$ & & & & & 出 & & & & & & & & & 出 & & & & & 出 \\
\hline 嵓 & & & & & & & 嵩 & & & & & & & & & & & & & & 容 \\
\hline & & & & & & & & & & & & & & & & & & & & & \\
\hline & & & & & & & 포 & & & & & & & & & & & & & & \\
\hline$\sum_{4}$ & & & & & & & $\sum_{\leq}$ & & & & & & & & & $\sum_{\leq}$ & & & & & $\sum_{\leq}$ \\
\hline 忿 & & & & & & & 忿 & & & & & & & & & & & & & & $\stackrel{9}{\mathcal{Z}}$ \\
\hline & & & & & & & & & & & & & & & & & & & & & \\
\hline ż & & & & & & & za & & & & & & & & & za & & & & & $\underset{\alpha}{z}$ \\
\hline$\tilde{O}$ & & & & & & & $\tilde{\mathrm{O}}$ & & & & $\tilde{O}$ & & & & & & & $\tilde{O}$ & & & \\
\hline & & & & & & & & & & & & & & & & & & & & & \\
\hline & & & & & & & & & & & & & & & & & & & & & \\
\hline & & & & & & & & & & & & & & & & & & & & & \\
\hline & & & & & & & $\stackrel{\$}{\mathrm{~S}}$ & & & & & & & & & & & & & & 今 \\
\hline 그 & & & & & & & & & & & & & & & & & & & & & \\
\hline 䏒 & & 离 & & & & & 宏 & & 舀 & & 离 & 离 & 离 & 舀 & & $\stackrel{\text { 宸 }}{ }$ & 蔍 & 离 & & & 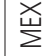 \\
\hline 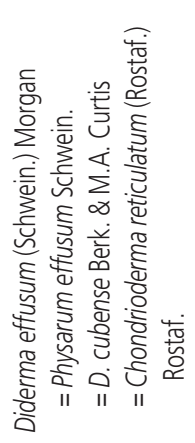 & 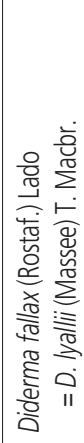 & 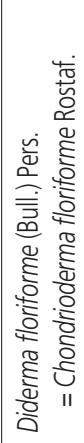 & 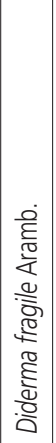 & 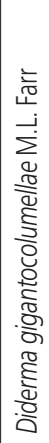 & 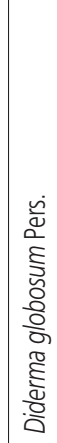 & 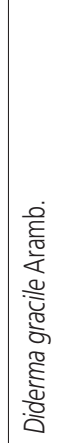 & 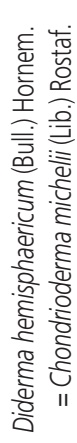 & 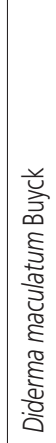 & 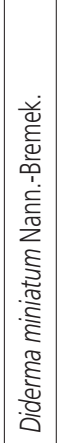 & 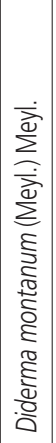 & 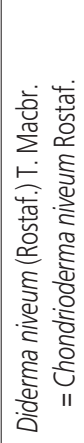 & 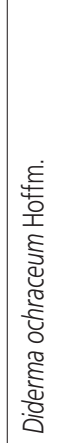 & 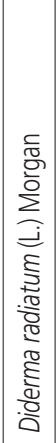 & 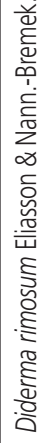 & 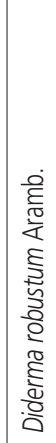 & 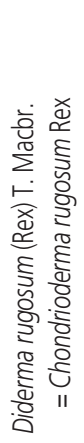 & 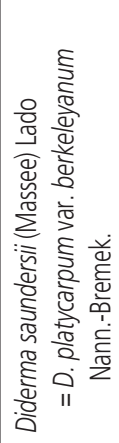 & 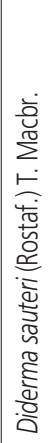 & 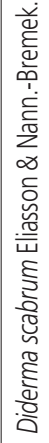 & 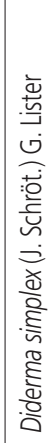 & 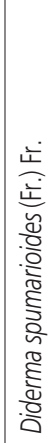 \\
\hline
\end{tabular}




\begin{tabular}{|c|c|c|c|c|c|c|c|c|c|c|c|c|c|c|c|c|c|c|c|c|c|}
\hline- & - & $\sim$ & $\sim$ & $\infty$ & $\sim$ & - & a & $N$ & - & in & - & $\div$ & - & - & Ln & \pm & in & - & - & $m$ & n \\
\hline & 要 & & & & & & $\begin{array}{l}\mathbb{1} \\
\text { S }\end{array}$ & & & & & 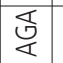 & & & & 委 & 委 & & & 委 & 委 \\
\hline & & & $\bar{U}$ & $\bar{U}$ & & & $\bar{J}$ & & & & & & & & & こ & & & & & \\
\hline & & & & & & & & & & & & & & & & $\overrightarrow{\stackrel{P}{y}}$ & & & & & \\
\hline & & & & & & & & & & & & & & & & 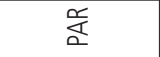 & & & & & \\
\hline & & & & & & & & & & & & $\vec{\Phi}$ & & & $\vec{\circ}$ & $\vec{\varnothing}$ & & & & & \\
\hline & & & & & & & & & & & & & & & & & & & & & \\
\hline & & & & & & & 己. & 己己 & & 己. & & 己⿱ユ口 & & & & 己્u & & & & 己.ّ & \\
\hline 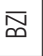 & & & & $\bar{\aleph}$ & & & $\bar{\infty}$ & & $\bar{\nwarrow}$ & হ্ল & & $\bar{\aleph}$ & হ্ল & & & న్্ & & & $\bar{\triangleright}$ & & হ্ল \\
\hline & & & & & & & & & & & & & & & & & & & & & \\
\hline & & & & & & & & & & & & & & & & & & & & & \\
\hline & & & & & & & & & & & & & & & & & & & & & \\
\hline & & 妾 & & & & & & & & Z & & 妾 & & & $\sim$ & Z & Z & & & Z & \\
\hline & & & & & & & $\sum$ & & & $\sum$ & & $\sum$ & & & & $\sum$ & $\sum$ & & & & $\sum_{j}$ \\
\hline & & & & & & & 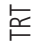 & & & & & 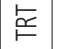 & & & & & & & & & \\
\hline & & & & & & & & & & & & $\frac{2}{3}$ & & $\frac{2}{3}$ & $\frac{2}{3}$ & ऐ & & & & & $\frac{z}{3}$ \\
\hline & & & & 岃 & & & & & & & & 出 & & & & & & & & & \\
\hline & & & & & & & 嵓 & & & & & 嵓 & & & & & & & & & \\
\hline & & & & ¿ & & & & & & & & & & & & & & & & & \\
\hline & & & & & & & & & & & & 氶 & & & & $\overline{\underline{X}}$ & & & & & \\
\hline & & & & $\sum$ & & & 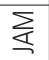 & & & & & 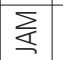 & & & & $\sum$ & & & & & $\sum_{i}$ \\
\hline & & & & ב⿱ ב⿱山⿻ & & & & & & & & ב⿱ & & & 学 & & & & & & \\
\hline & & & & & & & & & & & & & & & & & & & & & \\
\hline & & & & & & & & & & & & 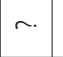 & & & & & & & & & $\sum_{\alpha}$ \\
\hline & & & & $\tilde{U}$ & & & & & & & & $\tilde{u}$ & & & $\tilde{u}$ & $\tilde{\varphi}$ & $\tilde{U}$ & & & & \\
\hline & & & & & & & & & & & & & & & & & & & & & $\frac{u}{z}$ \\
\hline & & & & & & & & & & & & & & & & & & & & & \\
\hline & & & & & & & & & & & & & & & & & & & & & \\
\hline & & & & & 今 & & & & & & & & & & & & & & & & \\
\hline & & & & & & & & & & & & 긍 & & & & & & & & & \\
\hline & & 㱞 & 离 & 层 & $\stackrel{\text { 崖 }}{ }$ & $\stackrel{\times}{\Sigma}$ & 宮 & 离 & & $\stackrel{x}{\Sigma}$ & 离 & 峃 & & & 离 & 离 & 离 & 羡 & & $\sim$ & \\
\hline 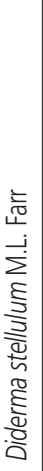 & 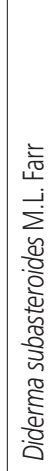 & 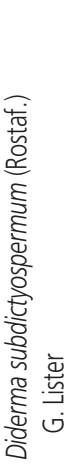 & 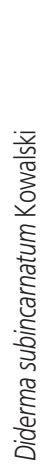 & 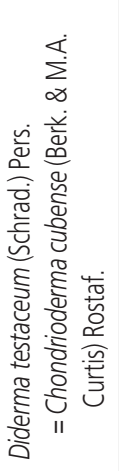 & 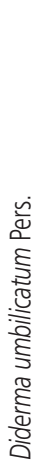 & 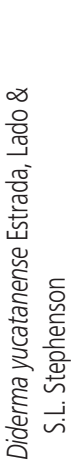 & 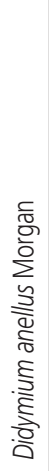 & 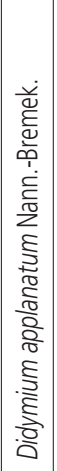 & 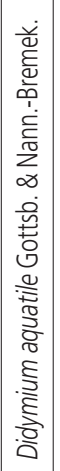 & 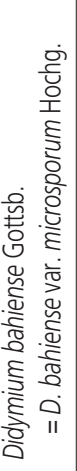 & 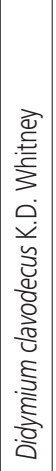 & 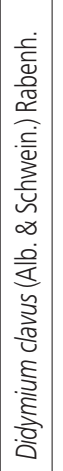 & 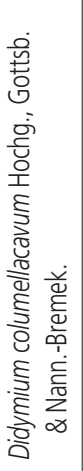 & 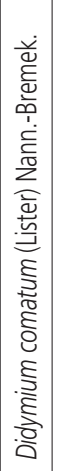 & 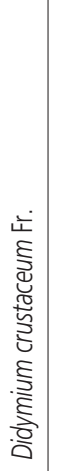 & 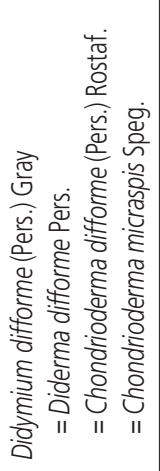 & 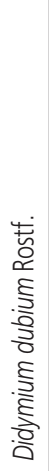 & 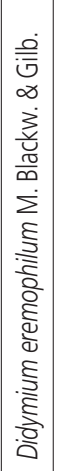 & 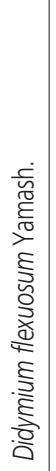 & 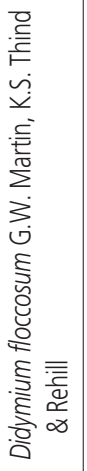 & 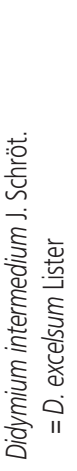 \\
\hline
\end{tabular}




\begin{tabular}{|c|c|c|c|c|c|c|c|c|c|c|c|c|c|c|c|c|c|c|c|c|}
\hline$\approx$ & - & - & $\sigma$ & $N$ & $N$ & $m$ & - & $=$ & $\bar{\sim}$ & $\theta$ & - & $\sim$ & - & - & - & $N$ & 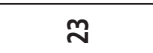 & - & - & - \\
\hline 委 & & & & & & 蛋 & & 蛋 & 委 & & & & 勇 & & & & 委 & & & \\
\hline $\bar{\nu}$ & & & & & & $\bar{\nu}$ & & $\bar{\nu}$ & $\bar{\nu}$ & & & & & & & & $\bar{\nu}$ & & & $\bar{U}$ \\
\hline \multirow[t]{2}{*}{ 록 } & & & & & & & & 록 & 곡 & & & & & & & & 곧 & & & \\
\hline & & & & & & & & & & & & & & & & & $\frac{\widetilde{c}}{\alpha}$ & & & \\
\hline \multirow[t]{2}{*}{ 요 } & & & & & & $\overrightarrow{\mathrm{D}}$ & & & $\vec{\varnothing}$ & & & & & & & & $\overrightarrow{\mathrm{O}}$ & & & \\
\hline & & & & & & & & & & & & & & & & & 畄 & & & \\
\hline $\mathrm{Z}_{\mathrm{U}}$ & 总 & & 급 & 己. & & 己. & & 总 & 겁 & 己. & & & & 忌 & & & $\mathrm{Z}_{\mathrm{U}}$ & & & \\
\hline $\bar{\triangleright}$ & & & & & $\bar{\infty}$ & $\overline{\aleph ্ ~}$ & & $\bar{\infty}$ & $\bar{\infty}$ & $\bar{\infty}$ & & & & & & & $\bar{\aleph}$ & & & \\
\hline \multirow[t]{3}{*}{ 마⿺ } & & & & & & & & & & & & & & & & & & & & \\
\hline & & & & & & & & & & & & & & & & & & & & \\
\hline & & & & & & & & & 产 & & & & & & & & & & & \\
\hline 妾 & & & & & & 妾 & & & 妾 & & & & & & & & 变 & & & \\
\hline$\sum_{j}$ & & & $\sum$ & & & & & $\sum_{\mathcal{U}}$ & $\sum_{j}$ & & & & & & & & $\sum_{j}$ & & & \\
\hline 占 & & & & & & & & & 茞 & & & & & & & & 占 & & & \\
\hline 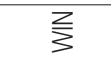 & & & & & & 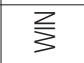 & & 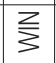 & 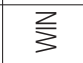 & & & & & & & & 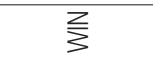 & & & \\
\hline 出 & & & & & & 出 & & 岃 & 岃 & & & & & & & & 出 & & & \\
\hline 岁 & & & & & & & & & 岁 & $\sim$ & & & & & & & 容 & & & \\
\hline$\overline{0}$ & & & & & & $\sum_{0}$ & & & $\overline{0}$ & & & & & & & & $\overline{0}$ & & & \\
\hline 衫 & & & & & & & & & 㕵 & & & & & & & & 㕵 & & & \\
\hline$\sum_{\leq}$ & & & $\underset{3}{\sum}$ & & & $\underset{\$}{2}$ & & $\underset{\Sigma}{i}$ & $\underset{1}{2}$ & & & & & & & & $\sum_{\$}$ & & & \\
\hline \multirow[t]{2}{*}{$\stackrel{9}{\mathrm{u}}$} & & & & & & 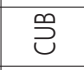 & & & & & & & & & & & $\stackrel{\rho}{\mathcal{Z}}$ & & & \\
\hline & & & & & & & & & & & & & & & & & 焉 & & & \\
\hline 亮 & & & & & & & & & 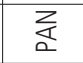 & & & & & & & & 交 & & & \\
\hline$\tilde{O}$ & & & & & & & & $\tilde{O}$ & $\tilde{O}$ & $\tilde{O}$ & & $\check{\mathcal{U}}$ & & & & & $\tilde{O}$ & & & \\
\hline$\frac{u}{z}$ & & & & & & & & & & & & & & & & & $\frac{u}{z}$ & & & \\
\hline & & & & & & & & & & & & & & & & & & & & \\
\hline \multirow[t]{3}{*}{ 오 } & & & & & & 호 & & & 오 & & & & & & & & & & & \\
\hline & & & & & & & & & $\stackrel{\mathbb{S}}{\mathrm{S}}$ & & & & & & & & & & & \\
\hline & & & & & & & & & & & & & & & & 금 & & & & \\
\hline 离 & & 离 & 离 & 盖 & 寽 & 离 & 羡 & 站 & 离 & 岀 & 痥 & 离 & & & 离 & 岀 & 岀 & 岀 & 羡 & \\
\hline 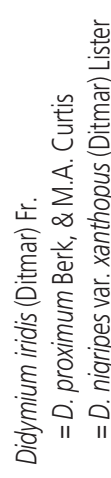 & 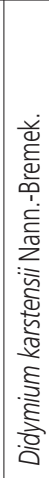 & 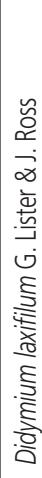 & 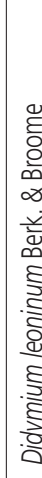 & 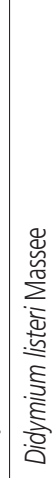 & 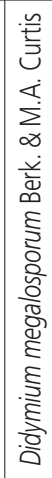 & 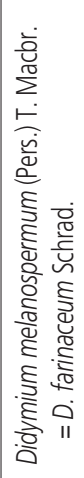 & 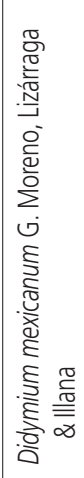 & 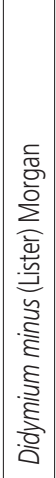 & 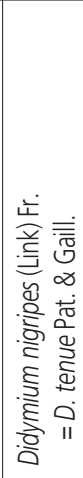 & 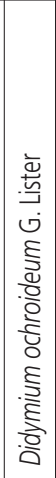 & 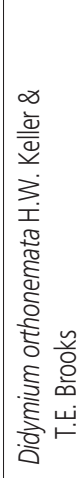 & 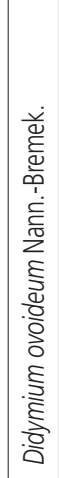 & 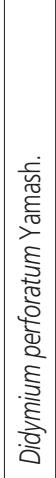 & 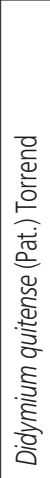 & 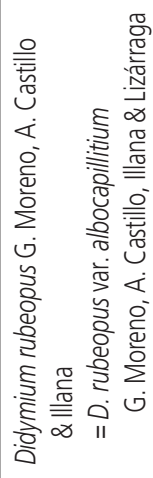 & 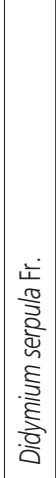 & 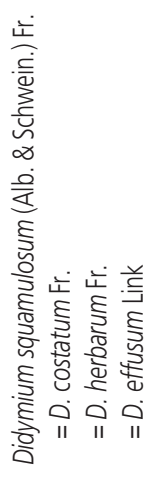 & 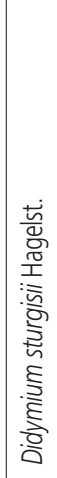 & 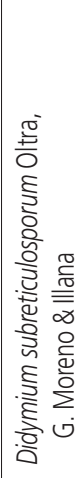 & 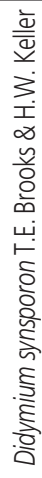 \\
\hline
\end{tabular}




\begin{tabular}{|c|c|c|c|c|c|c|c|c|c|c|c|c|c|c|c|c|c|c|c|c|c|c|c|}
\hline- & - & - & $\sigma$ & $\sigma$ & - & $N$ & $m$ & $\sim$ & - & - & $m$ & - & - & m & $N$ & \pm & $\sim$ & in & $\infty$ & - & Ln & $\sim$ & ㄱ. \\
\hline & & & & & & & & & & & & & & & & & & 氐 & 委 & & 芯 & & 氐 \\
\hline & & & $\bar{U}$ & & & & & & & & & & & & $\bar{U}$ & & & 己े & & & & & 己 \\
\hline & & & 곺 & & & & & & & & & & & & & & & & & & & & 롶 \\
\hline & & & & & & & & & & & & & & & & & & & & & & & $\frac{\mathfrak{c}}{a}$ \\
\hline & & & & & & & & & & & & & & & & & & & & & & & $\overrightarrow{\mathrm{D}}$ \\
\hline & & & & & & & & & & & & & & & & 啋 & & & & & & & \\
\hline & & & & 겁 & & 겄 & 己. & & & & & & & & & 己. & & 己. & & & & & 己ِ \\
\hline & & & & $\sim$ & & & $\bar{\aleph}$ & & & & $\bar{\aleph}$ & & & & & $\bar{\infty}$ & & $\bar{\infty}$ & $\bar{\infty}$ & & $\bar{\aleph}$ & & $\bar{\infty}$ \\
\hline & & & & & & & & & & & & & & & & & & & & & & & $\underset{\Psi}{\mathscr{W}}$ \\
\hline & & & & & & & & & & & & & & & & & & & & & & & \\
\hline & & & & & & & & & & & & & & & & & & & & & & & \\
\hline & & & 妾 & $\sim$ & & & & & & & & & & & & & & & & & & 永 & 妾 \\
\hline & & & & $\sum$ & & & & & & & & & & & & $\sum$ & & & & & & & \\
\hline & & & & & & & & & & & & & & & & 参 & & & & & & & 店 \\
\hline & & & & & & & & & & & & & $\sum_{3}$ & 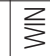 & & $\sum$ & & & 引 & & & & $\underline{z}$ \\
\hline & & & & & & & & & & & & & & & & 岃 & & & 岃 & & & & 岃 \\
\hline & & & & & & & & & & & & & & & & 岁 & & & & & & & 岁 \\
\hline & & & & & & & & & & & & & & & & & & & & & & & 음 \\
\hline & & & & & & & & & & & & & & & & & & & & & & & \\
\hline & & & & & & & & & & & & & & $\sum_{\leq}$ & & $\sum_{i}$ & & & $\sum_{\leq}$ & & & & 交 \\
\hline & & & & & & & & $\stackrel{\rho}{\mathcal{U}}$ & & & & & & & & $\stackrel{m}{\mathcal{Z}}$ & & & 忩 & & & 学 & 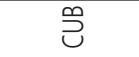 \\
\hline & & & & & & & & & & & & & & & & & & & & & & & \\
\hline & & & & $z_{a}$ & & & & & & & & & & & & 交 & & & & & & & ż \\
\hline & & & & & & & & 号 & & & & & & & & $\tilde{o}$ & & & $\tilde{O}$ & & $\stackrel{\sim}{\cup}$ & & $\tilde{o}$ \\
\hline & & & & & & & & & & & & & & & & & & & & & & & $\frac{u}{z}$ \\
\hline & & & & & & & & & & & & & & & & & & & & & & & \\
\hline & & & & & & & & & & & & & & & & & & & & & & & \\
\hline & & & & & & & & & & & & & & & & & S & & & & 吾 & & \\
\hline & & & & & & & & & & 그 & 그 & & & & & 프 & & & & & & & \\
\hline 离 & 齐 & 离 & 盖 & 隹 & 应 & 离 & 层 & & 离 & & 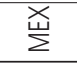 & 层 & & 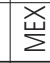 & 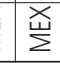 & 厹 & 离 & 离 & 嵀 & 㠍 & 售 & & 虔 \\
\hline 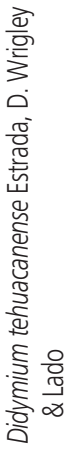 & 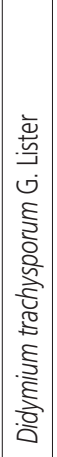 & 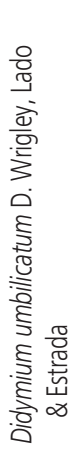 & 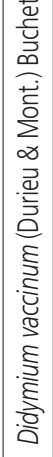 & 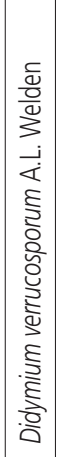 & 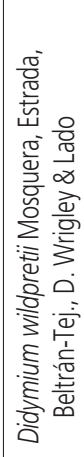 & 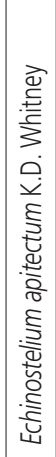 & 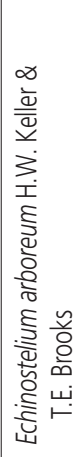 & 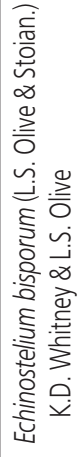 & 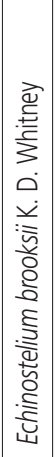 & 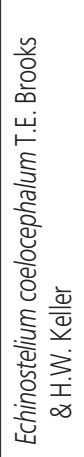 & 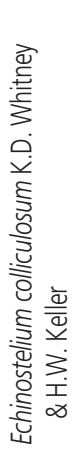 & 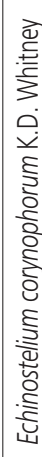 & 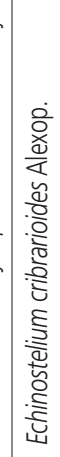 & 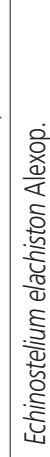 & 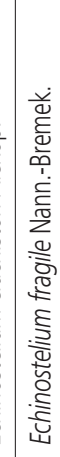 & 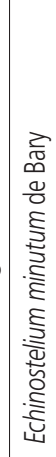 & 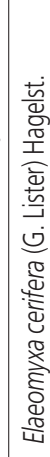 & 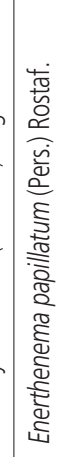 & 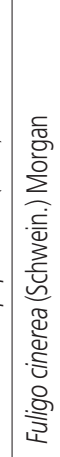 & 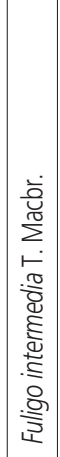 & 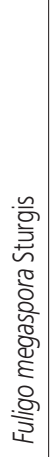 & 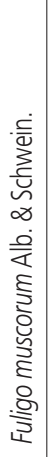 & 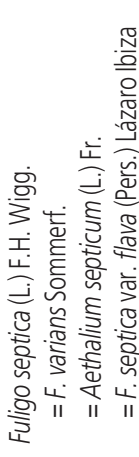 \\
\hline
\end{tabular}




\begin{tabular}{|c|c|c|c|c|c|c|c|c|c|c|c|c|c|}
\hline- & $\approx$ & $\stackrel{\text { ㅁ }}{\sim}$ & - & $N$ & $\infty$ & $\sim$ & n & - & $N$ & 6 & - & $\bar{\sim}$ & 0 \\
\hline \multirow[t]{30}{*}{ 委 } & 昏 & 㜽 & & & & & & & & & & 昏 & 昏 \\
\hline & $\bar{U}$ & $\bar{J}$ & & & & & $\bar{U}$ & & & & & $\bar{U}$ & \\
\hline & & 록 & & & & & & & & & & 록 & \\
\hline & 営 & & & & & & & & & & & & \\
\hline & & & & & & & & & & & & $\vec{\varnothing}$ & \\
\hline & 㗂 & & & & & & & & & & & & \\
\hline & 己. & 己己 & & & & & & & & 己. & & 겁 & \\
\hline & $\bar{\varnothing}$ & $\bar{\triangleright}$ & $\bar{\infty}$ & & $\bar{\varnothing}$ & & $\bar{\varnothing}$ & & & $\bar{\aleph}$ & & $\bar{\aleph}$ & $\bar{\aleph}$ \\
\hline & $\underset{ષ}{\mathscr{4}}$ & & & & & & & & & & & $\underset{ષ}{\mathscr{4}}$ & \\
\hline & & & & & & & & & & & & & \\
\hline & & & & & & & & & & & & & \\
\hline & 妾 & 急 & & 妾 & & 萝 & & & & & & 妾 & \\
\hline & 效 & $\sum$ & & & $\sum$ & & & & & & & 主 & \\
\hline & 占 & $\stackrel{\underline{E}}{\underline{E}}$ & & & & & & & & & & 占 & \\
\hline & $\frac{2}{3}$ & 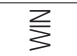 & & & $\sum_{3}$ & & $\frac{z}{3}$ & & $\frac{z}{3}$ & & & $\frac{2}{3}$ & \\
\hline & 出 & 岃 & & & & & & & & & & 岜 & \\
\hline & 容 & 亗 & & & & & & & & 容 & & 容 & 容 \\
\hline & $\bar{O}$ & 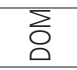 & & & & & & & & & & ¿ & ¿ \\
\hline & & & & & & & & & & & & & \\
\hline & $\sum_{\leq}$ & & & & & & & & $\sum_{\leq}$ & & & $\sum_{\leq}$ & $\sum_{\leq}$ \\
\hline & $\stackrel{9}{己}$ & $\stackrel{9}{己}$ & & & $\stackrel{9}{己}$ & & & & & $\stackrel{9}{己}$ & & $\stackrel{9}{\mathcal{Z}}$ & \\
\hline & & 昰 & & & & & & & & & & & \\
\hline & 衣 & $\sim$ & & & 衣 & & 交 & & & & & za & \\
\hline & $\check{\mathcal{U}}$ & & & & $\widetilde{o}$ & & $\widetilde{O}$ & & & $\widetilde{o}$ & & $\tilde{\mathrm{U}}$ & \\
\hline & $\triangleq$ & $\sim$ & & & & & & & & & & $\triangleq$ & \\
\hline & & & & & & & & & & & & & \\
\hline & 호 & & & & & & & & & & & & \\
\hline & 売 & & & & & & & & & & & 勇 & \\
\hline & & & & & 구 & & 그 & & & & & & \\
\hline & 离 & 崖 & & 峃 & 离 & 学 & 崖 & 峃 & & 离 & 岏 & 妵 & 离 \\
\hline 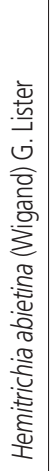 & 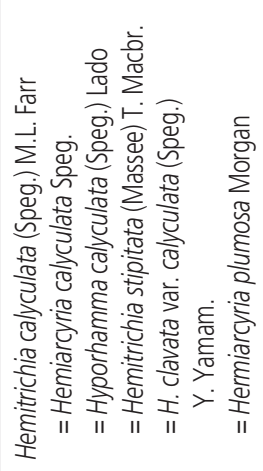 & 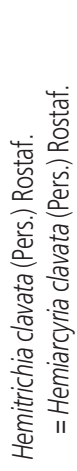 & 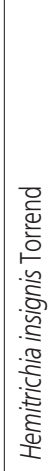 & 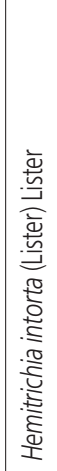 & 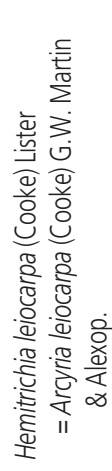 & 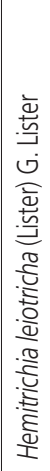 & 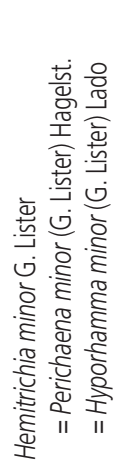 & 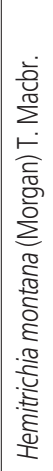 & 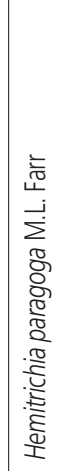 & 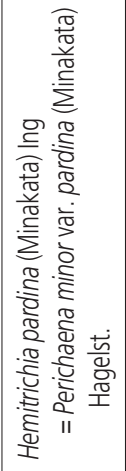 & 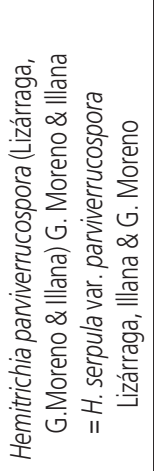 & 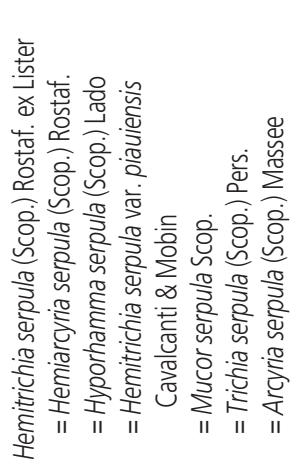 & 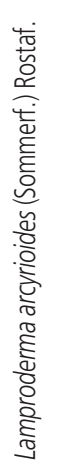 \\
\hline
\end{tabular}




\begin{tabular}{|c|c|c|c|c|c|c|c|c|c|c|c|c|c|c|c|c|c|c|c|c|c|c|}
\hline$\sim$ & - & $N$ & - & in & - & \pm & - & 0 & - & $\sim$ & - & - & - & a & - & $\sim$ & m & $m$ & - & $m$ & - & $m$ \\
\hline & & & & & & & & $\underset{4}{\mathbb{1}}$ & $\underset{4}{\mathbb{1}}$ & $\underset{\mathbb{Z}}{\mathbb{J}}$ & & 芯 & & & & & & & & & & \\
\hline & & & & & & & & $\bar{\nu}$ & & $\bar{U}$ & & & & $\bar{\Xi}$ & & & & & & & & \\
\hline & & & & & & & & & & & & & & & & & & & & & & $\overrightarrow{\vec{y}}$ \\
\hline & & & & & & & & & & & & & & & & & & & & & & \\
\hline & & & & & & $\vec{D}$ & & & & & & & & 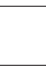 & & & & & & & & \\
\hline & & & & & & & & & & & & & & & & & & & & & & \\
\hline & & & & $\sim$ & & 芭 & & & & & & & & 己. & & & & & & & & \\
\hline & & & & $\bar{\infty}$ & & $\bar{ळ}$ & & $\bar{\infty}$ & & & & & & $\bar{\Phi}$ & & $\bar{\infty}$ & $\bar{\aleph}$ & $\bar{\infty}$ & & $\bar{\infty}$ & & \\
\hline & & & & & & & & & & & & & & 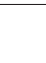 & & & & & & & & \\
\hline & & & & & & & & & & & & & & 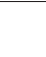 & & & & & & . & & \\
\hline & & & & & & & & & & & & & & 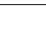 & & & & & & & & \\
\hline & & & & 妾 & & 壱 & & & & & & & & & & & & & & & & \\
\hline & & & & $\sum_{U}$ & & $\sum_{U}$ & $\sum_{U}$ & $\sum_{U}$ & & & & & & $\sum_{\mathcal{U}}$ & & & & & & & & \\
\hline & & & & & & & & & & & & & & & & & & & & & & \\
\hline & & & & & & 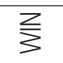 & & & & & & & & & & & & & & & & \\
\hline & & & & & & 岃 & & & & & & & & 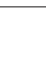 & & & & & & & & \\
\hline & & & & & & 容 & & & & & & & & & & & & & & & & \\
\hline & & & & & & & & & & & & & & . & & & & & & & & \\
\hline & & & & & & 㕵 & & & & & & & & & & & & & & & & \\
\hline & & & & & & 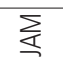 & & & & & & & & $\sum$ & & & & & & & & \\
\hline & & & & & & $\stackrel{9}{\mathrm{O}}$ & & & & & & & & $\stackrel{9}{\mathcal{Y}}$ & 急 & & & 孚 & & & & \\
\hline & & & & & & & & & & & & & & & & & & & & & & \\
\hline & & & & & & 歪 & & & & & & & & & & & & & & & & za \\
\hline$\tilde{\mathcal{O}}$ & $\widetilde{O}$ & $\tilde{O}$ & & $\tilde{O}$ & $\tilde{U}$ & $\tilde{O}$ & & $\tilde{O}$ & & & & & & $\tilde{U}$ & & & & & & & & \\
\hline
\end{tabular}

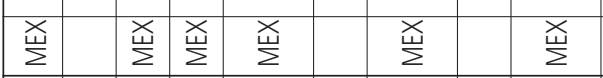

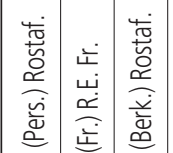

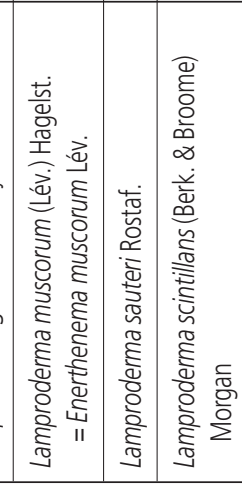

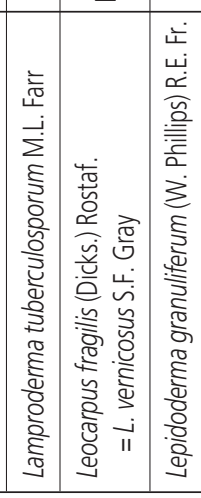

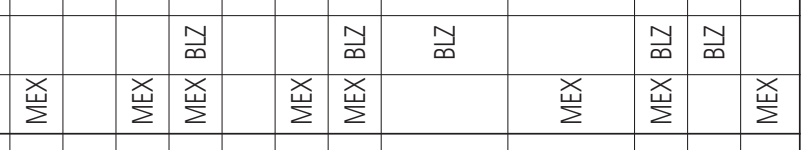

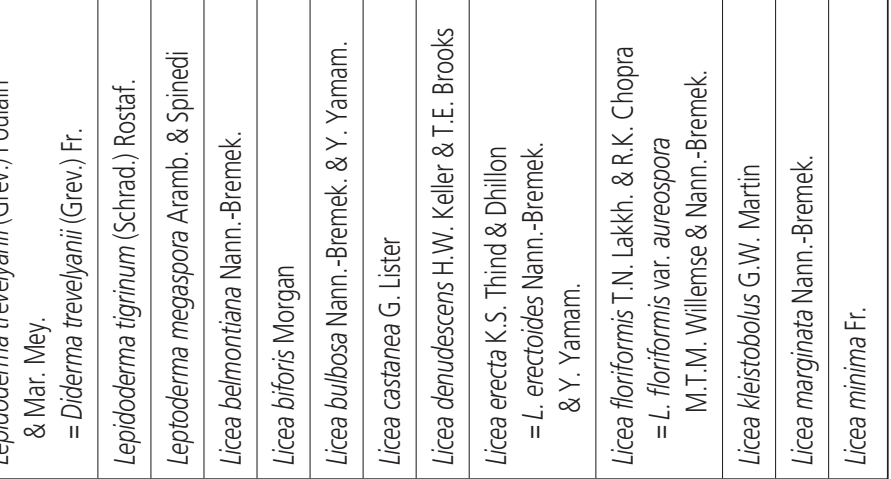




\begin{tabular}{|c|c|c|c|c|c|c|c|c|c|c|c|c|c|c|c|c|c|c|c|c|c|c|c|c|}
\hline- & 으 & $m$ & 6 & $\theta$ & - & $N$ & $N$ & $m$ & - & - & Ln & $m$ & $m$ & - & $m$ & $\sim$ & - & $r$ & ని & \pm & Ln & $m$ & $m$ & - \\
\hline & & & & & & & & & & & & & & & & 委 & & & 勇 & & 袋 & & & \\
\hline & & & & & & & & & & & & $\bar{J}$ & & & $\bar{U}$ & & & & 己 & & & & & \\
\hline & 록 & & & & & & 록 & & & & & & $\overrightarrow{\vec{g}}$ & & & & & & 록 & & $\overrightarrow{\vec{g}}$ & & & \\
\hline & & & & & & & & & & & & & & & & & & & & & & & & \\
\hline & & & & & & & & & & & & & & & & & & & & & & & & \\
\hline & 畄 & & & & & & & & & & 哹 & & & & & & & & & & & & & \\
\hline & 总 & & 곴 & 恙 & & & & & & & 겁 & 겁 & & & & & 겁 & & 겁 & 겁 & & 总 & 己 & \\
\hline & $\bar{\infty}$ & $\bar{\infty}$ & $\bar{\infty}$ & & & & & & & & & & $\bar{\infty}$ & & $\bar{্}$ & & & $\bar{\aleph}$ & $\bar{\aleph}$ & $\bar{\aleph}$ & $\bar{\infty}$ & & & \\
\hline & & & & & & & & & & & & & & & & & & & $\underset{\leftarrow}{\mathscr{Y}}$ & $\underset{\Psi}{\Psi}$ & & & & \\
\hline & & & & & & & & & & & & & & & & & & & & & & & & \\
\hline & & & & & & & & & & & & & & & & & & & & క̀ & & & & \\
\hline & Z & & & & & & & & & & & & & & & & & & Z & Z & & & & \\
\hline & & & & & & & & & & & & & & & & & & & $\sum_{\mathcal{U}}$ & $\sum_{\mathcal{U}}$ & & & & \\
\hline & & & & & & & & & & & & & & & & & & & 占 & & & & & \\
\hline & $\frac{Z}{3}$ & & $\frac{z}{3}$ & & & & & & & & & & & & & & & & $\sum_{3}$ & $\sum_{3}$ & & & & \\
\hline & & & & & & & & & & & & & & & & & & 出 & 岃 & 岃 & & & & \\
\hline & 岁 & & 岁 & & & & & & & & & & & & & & & & 容 & 岂 & & & & \\
\hline & & & & & & & & & & & & & & & & & & & 음 & & & & & \\
\hline & & & & & & & & & & & & & & & & & & & & & & & & \\
\hline & & & & & & & & $\sum_{\leq}$ & & & & & & & & & & $\gtreqless$ & $\sum_{\leq}$ & $\gtreqless$ & & & & \\
\hline & & & & & & & & & & & 总 & & & & & & & 总 & 党 & 学 & & & & \\
\hline & & & & & & & & & & & & & & & & & & & 焉 & & & & & \\
\hline & 齐 & & 充 & & & & & $z_{a}$ & & & & & & 齐 & & & & $z_{a}$ & 交 & $z_{a}$ & 齐 & & & \\
\hline & $\tilde{O}$ & & & $\tilde{\vartheta}$ & & & & & & & & & & & & & & & $\tilde{\vartheta}$ & $\tilde{\cup}$ & & $\tilde{\vartheta}$ & $\tilde{U}$ & \\
\hline & & & & & & & & & & & & & & & & & & $\frac{u}{z}$ & $\frac{u}{z}$ & & & & & \\
\hline & & & & & & & & & & & & & & & & & & & & & & & & \\
\hline & & & & & & & & & & & & & & & & & & & & & & & & \\
\hline & & & & & & & & & & & & & & & & & & & & & & & & \\
\hline & & 그 & & ले & & 그 & के & & & & ले & & & & & & & & & & & & & \\
\hline 层 & 离 & 离 & 离 & 离 & 离 & 离 & & 离 & 层 & 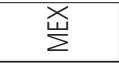 & 离 & 沗 & 离 & & 离 & 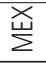 & & 离 & 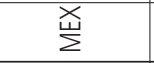 & 层 & 离 & 层 & 层 & 嵃 \\
\hline 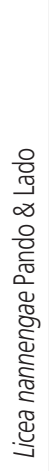 & 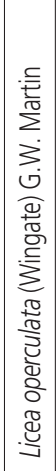 & 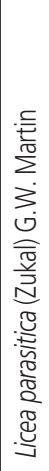 & 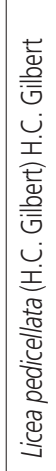 & 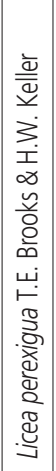 & 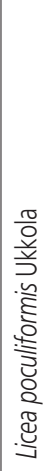 & 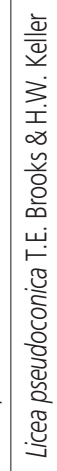 & 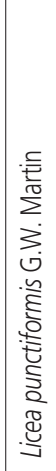 & 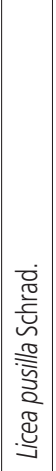 & 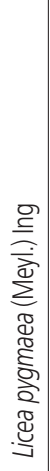 & 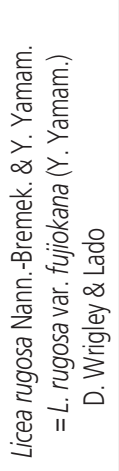 & 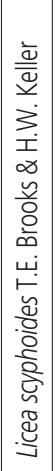 & 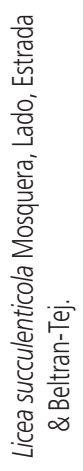 & 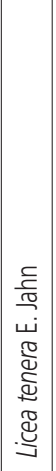 & 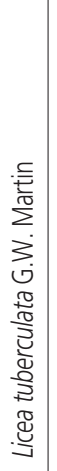 & 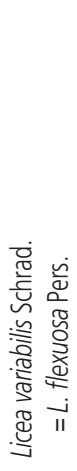 & 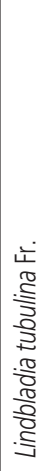 & 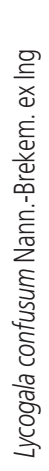 & 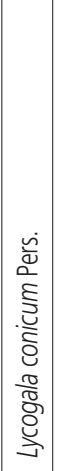 & 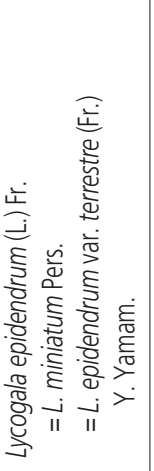 & 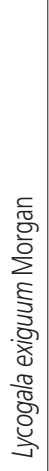 & 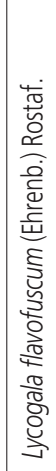 & 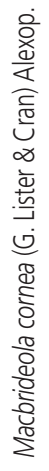 & 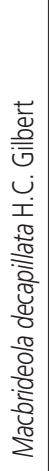 & 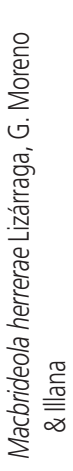 \\
\hline
\end{tabular}




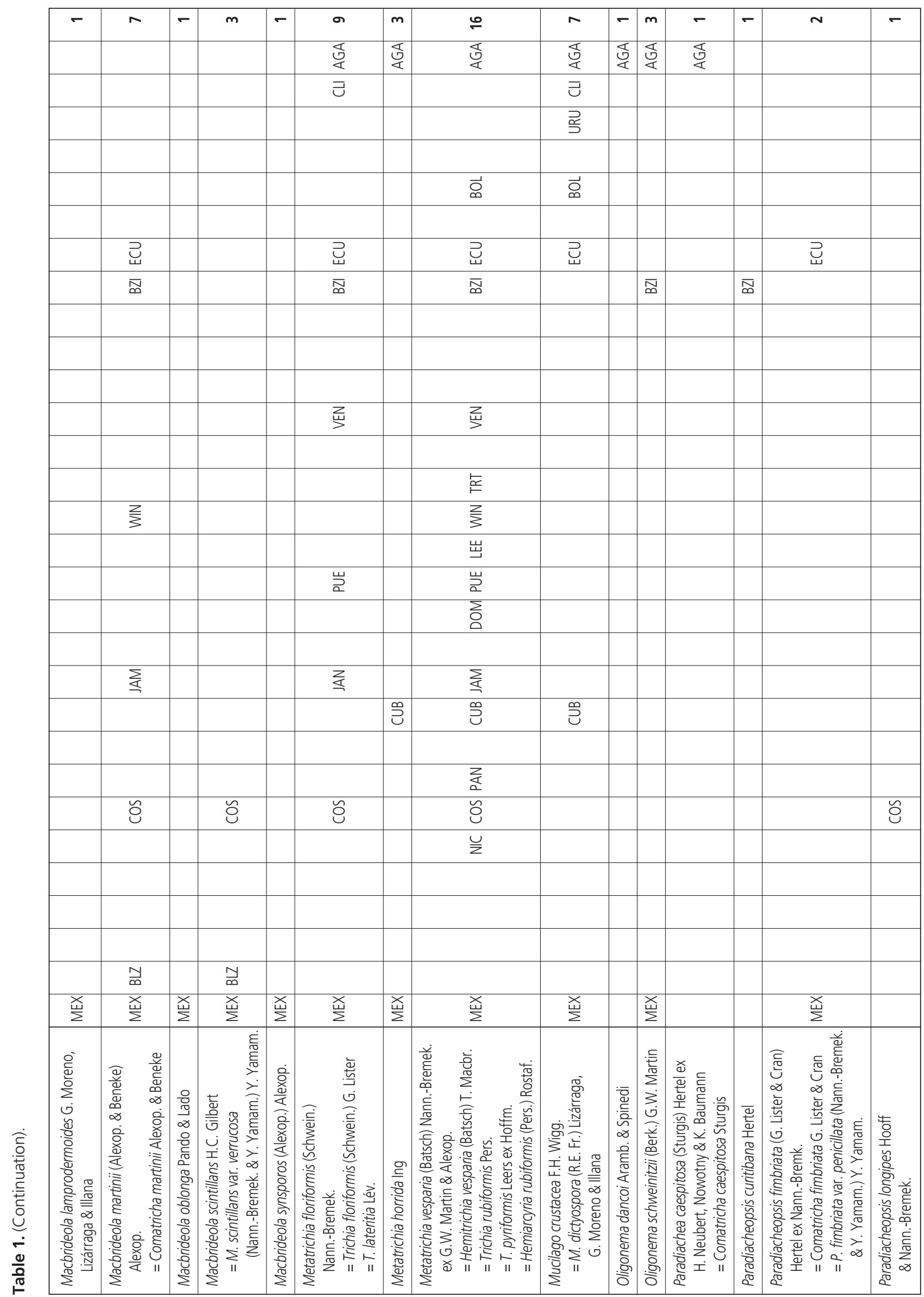




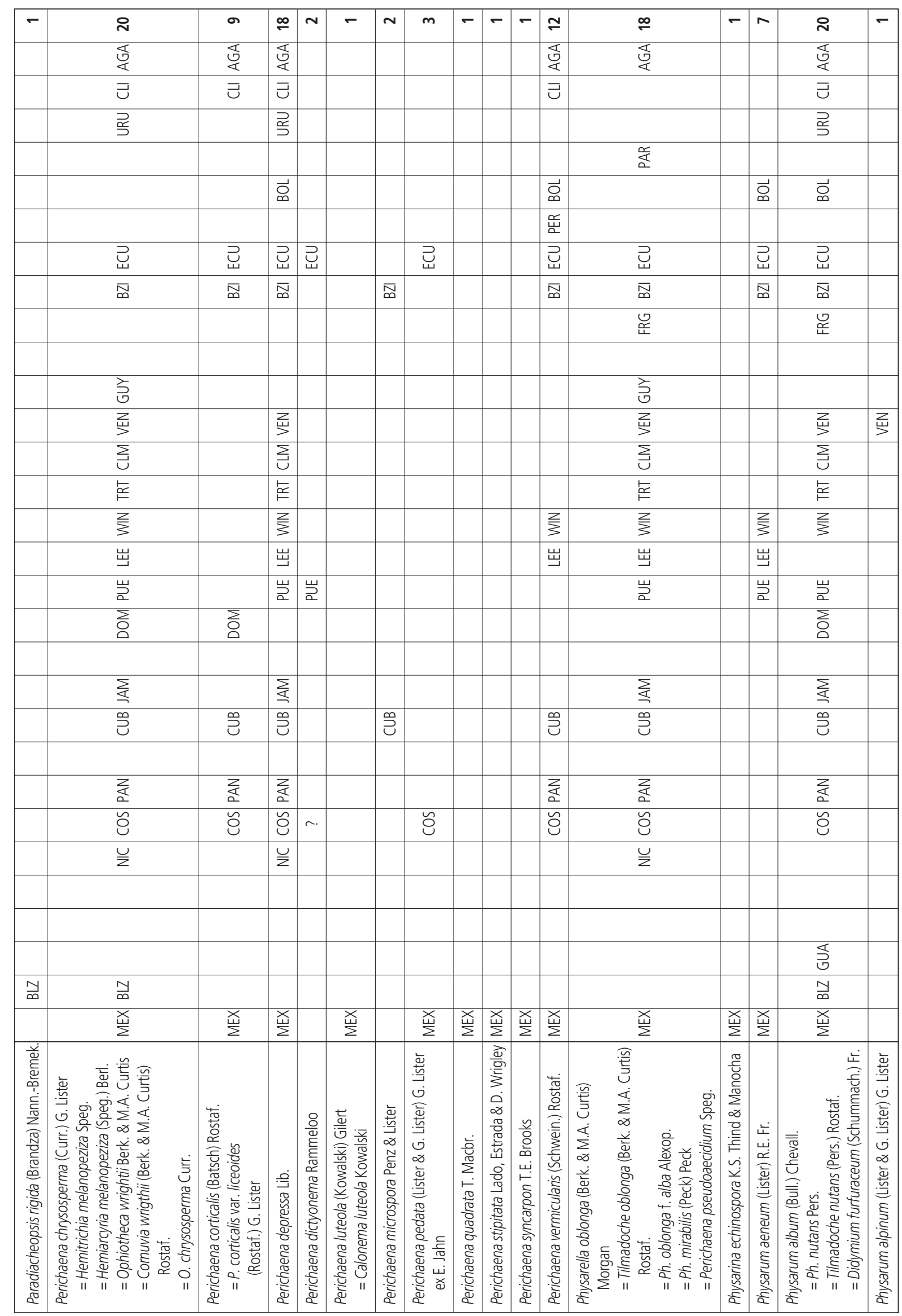




\begin{tabular}{|c|c|c|c|c|c|c|c|c|c|c|c|c|c|c|c|c|c|c|c|c|c|c|c|c|}
\hline$\sim$ & $\infty$ & $m$ & 0 & \pm & ని & $\sigma$ & - & - & $\approx$ & $\infty$ & $\stackrel{d}{d}$ & $\nabla$ & o & Ln & $\sim$ & $m$ & - & \pm & $\infty$ & $n$ & - & $r$ & $\infty$ & $\stackrel{2}{2}$ \\
\hline & & 哥 & & 正 & 蚝 & & & & 热 & 褰 & 委 & 霆 & & & & & 哥 & 志 & & & & & 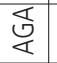 & 正 \\
\hline & & $\overrightarrow{\mathrm{U}}$ & & $\bar{U}$ & & $\bar{v}$ & & & $\bar{U}$ & $\bar{U}$ & $\bar{u}$ & & & & $\bar{U}$ & & & & $\bar{U}$ & $\bar{U}$ & & & & $\bar{U}$ \\
\hline & & & & & $\vec{P}$ & & & & 록 & & & & & & & & & & 랃 & & & & & \\
\hline & & & & & & & & & 孚 & & & & & & & & & & & & & & & \\
\hline & & & & & $\vec{\varnothing}$ & & & & ठ্口 & & ठ্口 & & & $\vec{\varnothing}$ & & & & & & & & & & \\
\hline & & & & 畄 & 㗂 & & & & & & 畄 & & & 畄 & & & & & & & & & & 絗 \\
\hline & & & & 己. & 己. & & & & 己ِ & & 겁 & & 겁 & & & & & 겄 & 己. & & & 己. & $\sim$ & 己. \\
\hline $\bar{\infty}$ & $\bar{\infty}$ & $\bar{\infty}$ & & $\bar{\infty}$ & $\bar{\infty}$ & & & & $\bar{\infty}$ & & $\bar{\aleph}$ & & $\bar{\infty}$ & $\bar{\infty}$ & & & & $\bar{\infty}$ & $\bar{\infty}$ & $\bar{\infty}$ & & $\bar{\infty}$ & $\bar{\infty}$ & $\bar{\infty}$ \\
\hline & & & & $\underset{\Psi}{\Psi}$ & $\underset{\Psi}{\Psi}$ & & & & & & 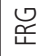 & & & & & & & & & & & & & $\underset{\Psi}{\Psi}$ \\
\hline & & & & & 寽 & & & & 兴 & & 孚 & & & & & & & & & & & & & \\
\hline & & & & & 空 & & & & & & & & & & & & & & & & & & & \\
\hline & Z & & Z & Z & Z & $\sim$ & & & Z & Z & Z & & & & Z & & & & & & & & & Z \\
\hline & & & $\sum$ & 记 & $\sum$ & $\sum$ & & & $\sum$ & $\sum$ & $\sum_{\mathcal{U}}$ & & & & & $\sum$ & & $\sum_{U}$ & & $\sum$ & & $\sum$ & $\sum$ & 记 \\
\hline & & & & & 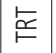 & & & & 店 & & $\stackrel{t}{\underline{E}}$ & & & & & & & 占 & & 虎 & & & & 扂 \\
\hline & $\sum_{3}$ & & & & $\sum_{3}$ & & 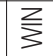 & & $\sum_{3}$ & $\frac{2}{3}$ & $\sum_{3}^{3}$ & & $\frac{2}{3}$ & & & & & $\sum_{3}$ & $\frac{2}{3}$ & & & $\frac{2}{3}$ & 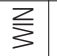 & \\
\hline & 岃 & & & 岃 & 出 & & & & 出 & 出 & 出 & & 出 & & & & & & 出 & 出 & & & & 岃 \\
\hline & 嵓 & & 亗 & & 岂 & & & & 嵓 & & 岁 & & 岁 & & & & & 容 & & & & 岁 & & \\
\hline & & & & & & & & & ¿ & & ¿o & & & & & & & ¿ & & & & & & \\
\hline & & & & 氶 & & & & & $\overline{\underline{x}}$ & & $\bar{X}$ & & & & & & & & & & & & & \\
\hline & & & $\sum_{\leq}$ & & $\sum_{\leq}$ & & & & $\sum$ & & $\sum_{\leq}$ & & & & & & & $\sum_{\leq}$ & $\sum_{\leq}$ & & & $\sum_{\leq}$ & $\sum_{4}$ & $\sum$ \\
\hline & & & & 学 & 荺 & & & & 学 & & 急 & & 学 & & & & & 党 & & & & & 忿 & \\
\hline & & & & & & & & & & & 焉 & & & & & & & & & & & & & 焉 \\
\hline & 交 & & & $\sum_{\alpha}$ & $\sum_{a}$ & & & & 交 & & 充 & & & & & & & 紊 & $\sum_{\alpha}$ & & & 交 & & 交 \\
\hline & $\sim$ & & $\tilde{U}$ & $\tilde{\varphi}$ & $\tilde{U}$ & $\tilde{O}$ & & & $\tilde{\circlearrowright}$ & $\tilde{U}$ & $\check{\theta}$ & $\tilde{U}$ & $\tilde{\cup}$ & $\tilde{U}$ & & $\tilde{u}$ & & $\tilde{O}$ & & $\sim$ & $\tilde{O}$ & & & $\tilde{\vartheta}$ \\
\hline & & & & & & & & & $\frac{u}{z}$ & & & $\frac{u}{z}$ & & & & & & & & & & & $\frac{U}{z}$ & \\
\hline & & & & & & & & & & & & & & & & & & & & & & & & \\
\hline & & & & & & & & & & & & & & & & & & & & & & & & \\
\hline & & & & & & & & & & & S & & & & & & & S & & & & & & \\
\hline & ్ㅡ & & & & & & & & & & 그 & & 그 & & & & & & & 그 & & & & \\
\hline 离 & 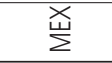 & & 层 & 层 & 离 & 离 & & 厹 & 訔 & 离 & 离 & 离 & 离 & 离 & & 离 & & 层 & & 层 & & & 希 & 层 \\
\hline 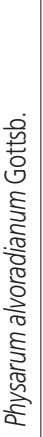 & 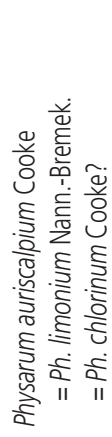 & 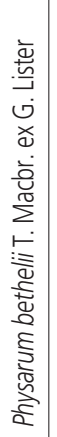 & 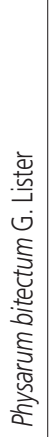 & 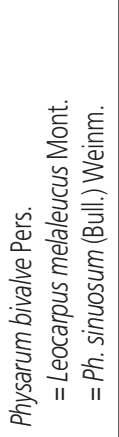 & 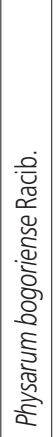 & 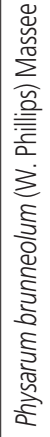 & 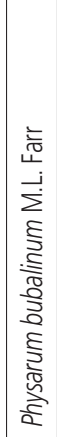 & 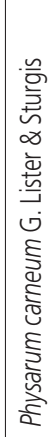 & 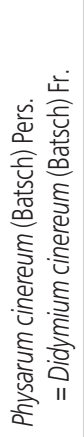 & 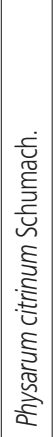 & 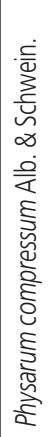 & 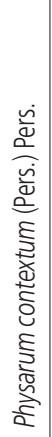 & 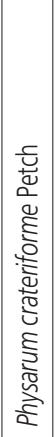 & 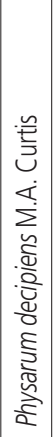 & 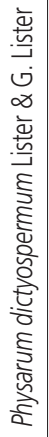 & 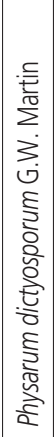 & 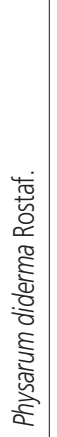 & 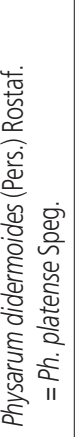 & 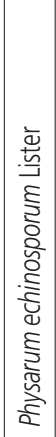 & 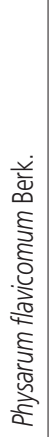 & 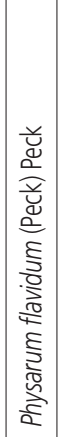 & 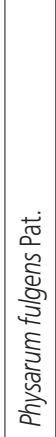 & 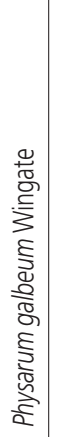 & 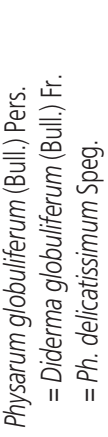 \\
\hline
\end{tabular}




\begin{tabular}{|c|c|c|c|c|c|c|c|c|c|c|c|c|c|c|c|c|c|c|c|c|c|}
\hline$\sigma$ & - & $\mp$ & - & in & 으 & 으 & $\sim$ & - & $m$ & $=$ & $N$ & $\sim$ & $\theta$ & - & a & - & $n$ & 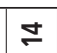 & - & a & + \\
\hline & & & & & 災 & 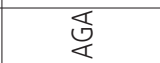 & & & & 㞼 & & & 㞼 & & & & 蛋 & 芯 & & & 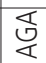 \\
\hline & & & & $\bar{U}$ & $\bar{U}$ & & & & $\bar{U}$ & & & & & & & & & & & & $\bar{U}$ \\
\hline 록 & & & & & & & & & & & & & & & & & & & & & \\
\hline & & & & & & $\frac{\alpha}{\alpha}$ & & & & & & & & & & & & & & & \\
\hline & & & & & & & & & & $\vec{\varnothing}$ & & & & & & & $\vec{D}$ & & & & \\
\hline & & & & & & & & & & & & & & & & & & & & & \\
\hline & & 己. & 己己 & & 己. & & & & & 己.ّ & & & & & & & & 己. & & 검 & \\
\hline $\bar{্}$ & & $\bar{\infty}$ & & $\bar{\aleph}$ & $\overline{\aleph ্}$ & $\bar{\aleph}$ & & & $\bar{\infty}$ & $\bar{\infty}$ & $\bar{\infty}$ & & $\overline{\aleph ্}$ & & $\bar{\infty}$ & $\bar{\aleph}$ & $\bar{\triangleright}$ & $\bar{\infty}$ & $\bar{\infty}$ & $\bar{\infty}$ & $\bar{\infty}$ \\
\hline & & 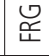 & & & & & & & & & & & & & & & & $\underset{\mathscr{K}}{\stackrel{\Psi}{*}}$ & & & \\
\hline & & & & & & & & & & & 受 & & & & & & & & & & \\
\hline & & & & & & & & & & & & & & & & & & & & & \\
\hline & & 埾 & & & $\sim$ & & & & & z & & & z & & & & & Z & & Z & \\
\hline$\sum_{U}$ & & $\sum$ & & & & $\sum_{U}$ & & & & $\sum_{\mathcal{U}}$ & & & & & & & & & & $\sum_{\mathcal{U}}$ & $\sum_{j}$ \\
\hline & & 占 & & & & & & & & 臣 & & & & & 占 & & & 占 & & & \\
\hline & & & & 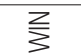 & 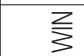 & 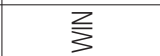 & & & & 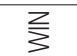 & & & & & & & 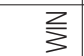 & ऐ & & ऐ & \\
\hline & & & & 岃 & 岃 & & & & & 岃 & & & & & & & & 岃 & & & \\
\hline$\sim$ & & 岁 & & & & & & & & 亗 & & & & & 容 & & & 容 & & & \\
\hline & & & & & $\overline{0}$ & & & & & $\overline{0}$ & & & & & & & & & & & \\
\hline & & & & & & & & & & & & & & & 至 & & & & & & \\
\hline & & $\sum_{\perp}$ & & & $\sum_{i}$ & $\sum_{i}$ & & & & $\sum_{i}$ & & & & & 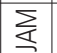 & & $\sum_{\leq}$ & $\sum_{i}$ & & $\sum_{\leq}$ & \\
\hline & & 愛 & & & 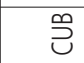 & & 紜 & & & $\stackrel{9}{\mathrm{Y}}$ & & & & & & & & $\stackrel{9}{\mathrm{~S}}$ & & & \\
\hline & & & & & & & & & & & & & & & & & & & & & \\
\hline & & & & & & 交 & & & & 交 & & & & & & & & & & $\underset{2}{2}$ & \\
\hline & & $\tilde{\mathcal{S}}$ & & & & $\tilde{ᄋ}$ & & & & $\widetilde{ᄋ}$ & & $\widetilde{ᄋ}$ & & & $\tilde{U}$ & & $\tilde{\mathrm{U}}$ & $\check{O}$ & & & \\
\hline & & & & & & & & & & & & & & & $\frac{u}{z}$ & & & $\frac{u}{z}$ & & & \\
\hline & & & & & & & & & & & & & & & & & & & & & \\
\hline & & & & & & & & & & & & & & & & & & & & & \\
\hline & & & & & & S & & & & S & & & & & & & & & & & \\
\hline & & & & & & & & & & & & & & & y & & & & & 그 & \\
\hline 离 & 厹 & 喥 & & 离 & 厹 & 厹 & 度 & 厹 & 厹 & 厹 & & 吝 & 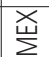 & 离 & 厹 & & 厹 & 厹 & & 离 & 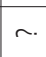 \\
\hline 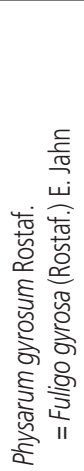 & 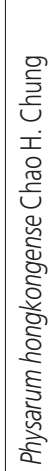 & 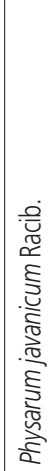 & 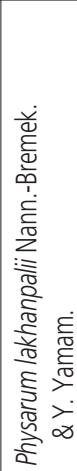 & 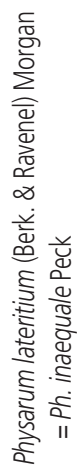 & 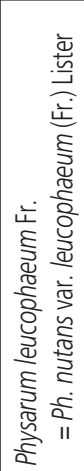 & 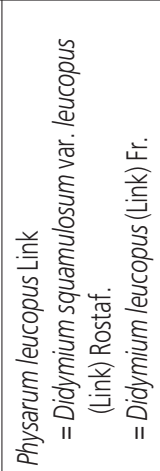 & 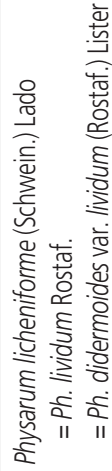 & 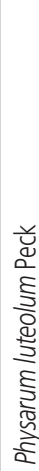 & 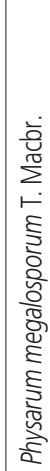 & 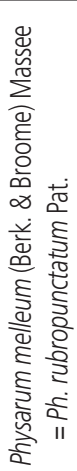 & 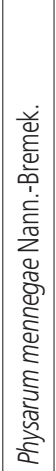 & 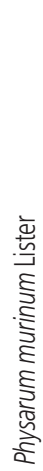 & 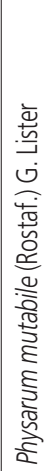 & 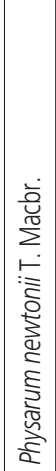 & 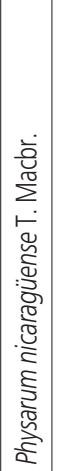 & 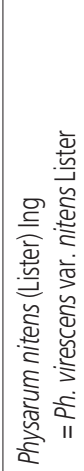 & 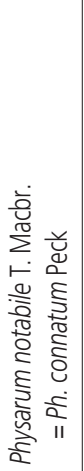 & 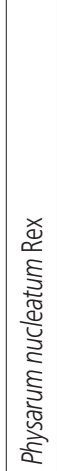 & 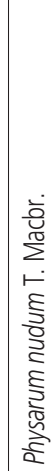 & 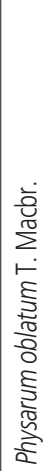 & 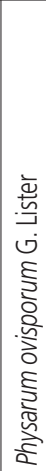 \\
\hline
\end{tabular}




\begin{tabular}{|c|c|c|c|c|c|c|c|c|c|c|c|c|c|c|c|c|c|}
\hline$\infty$ & $\sigma$ & \pm & in & - & r & g & N & $\infty$ & -6 & $m$ & $\sigma$ & $m$ & $\sim$ & 은 & $\sim$ & $n$ & r \\
\hline & 秀 & 重 & & & & 季 & & 高 & & & 胥 & & & & & 焉 & \\
\hline \multirow[t]{5}{*}{ J } & & & Ј & & & 己 & & & & & & & 己 & & ప & & \\
\hline & & $\overrightarrow{\vec{x}}$ & & & & $\overrightarrow{\underline{y}}$ & & $\overrightarrow{\vec{I}}$ & & & & & & & & & \\
\hline & & & & $\frac{\substack{a \\
\alpha}}{\alpha}$ & & & & & àd & & . & & & & & & \\
\hline & $\sim$ & & & & & $\vec{\Phi}$ & & & & & . & & & $\vec{\Phi}$ & & & \\
\hline & & & & & & 䍃 & & & & & & & & & & & 焉 \\
\hline$\sim$ & & & & & & 己 & & . & ( & & 忌 & $\sim$ & & 己 & & . & 己. \\
\hline $\bar{\Phi}$ & $\bar{\Phi}$ & $\bar{\Phi}$ & & & & $\bar{\Phi}$ & $\bar{\infty}$ & $\bar{\infty}$ & $\bar{a}$ & & $\bar{\aleph}$ & $\bar{\infty}$ & & $\bar{\Phi}$ & . & $\bar{\Phi}$ & $\sim$ \\
\hline \multirow[t]{3}{*}{$\stackrel{\mathscr{\Psi}}{\mathbb{4}}$} & & & & & & & & & & & & & & 茞 & & & \\
\hline & & 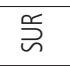 & & & & & & . & & & . & . & & & & . & \\
\hline & & 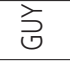 & & & & & & & & & & & & & & 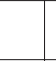 & \\
\hline \multirow[t]{3}{*}{ 吾 } & & & z & & 畜 & . & & . & 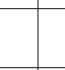 & & 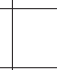 & z & & z & & Z & Z \\
\hline & & & 朊 & & & 就 & & ( & 5 & & . & 就 & & $\sum_{U}$ & & & \\
\hline & & $\stackrel{t}{\underline{\Phi}}$ & & & 占 & & & 点 & . & & 产 & & & 占 & & & \\
\hline \multirow[t]{5}{*}{ z } & & $\frac{2}{3}$ & $\underline{z}$ & & $\underline{z}$ & $\underline{z}$ & & & 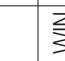 & $\frac{2}{3}$ & & & & $\underline{z}$ & & . & \\
\hline & & 岃 & & & & 岃 & & 岃 & & & 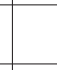 & & & 岃 & & 岃 & \\
\hline & r. & 訔 & & & & 嵩 & 訔 & 訔 & & 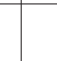 & 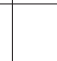 & & & 訔 & & 訔 & 嵩 \\
\hline & & & & & & $\overline{\mathrm{o}}$ & & & & & 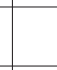 & & & & & & \\
\hline & & & & & & 포 & & . & & & 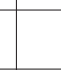 & & & & & . & $\bar{x}$ \\
\hline \multirow[t]{3}{*}{ 主 } & - & $\sum$ & & & $\frac{2}{3}$ & 交 & & $\frac{2}{3}$ & $\sum$ & $\sqrt{2}$ & $\sum_{x}$ & & & $\sum_{i}$ & & $\sum_{i}$ & \\
\hline & 唫 & $\stackrel{\rho}{\mathrm{U}}$ & & & & 学 & & & & & 今े & & & 学 & & & \\
\hline & & & & & & & & & & & & & & & & & \\
\hline \multirow[t]{3}{*}{ za } & & . & za & & zz & ż & & & & & 交 & & & 궁 & & & \\
\hline & & 茨 & & & 岁 & 岁 & & 岁 & r & & 茨 & & & $\tilde{o}$ & $\sim$ & & 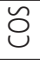 \\
\hline & & $\frac{u}{z}$ & & & & & & & & & & & & $\frac{u}{z}$ & & & \\
\hline
\end{tabular}

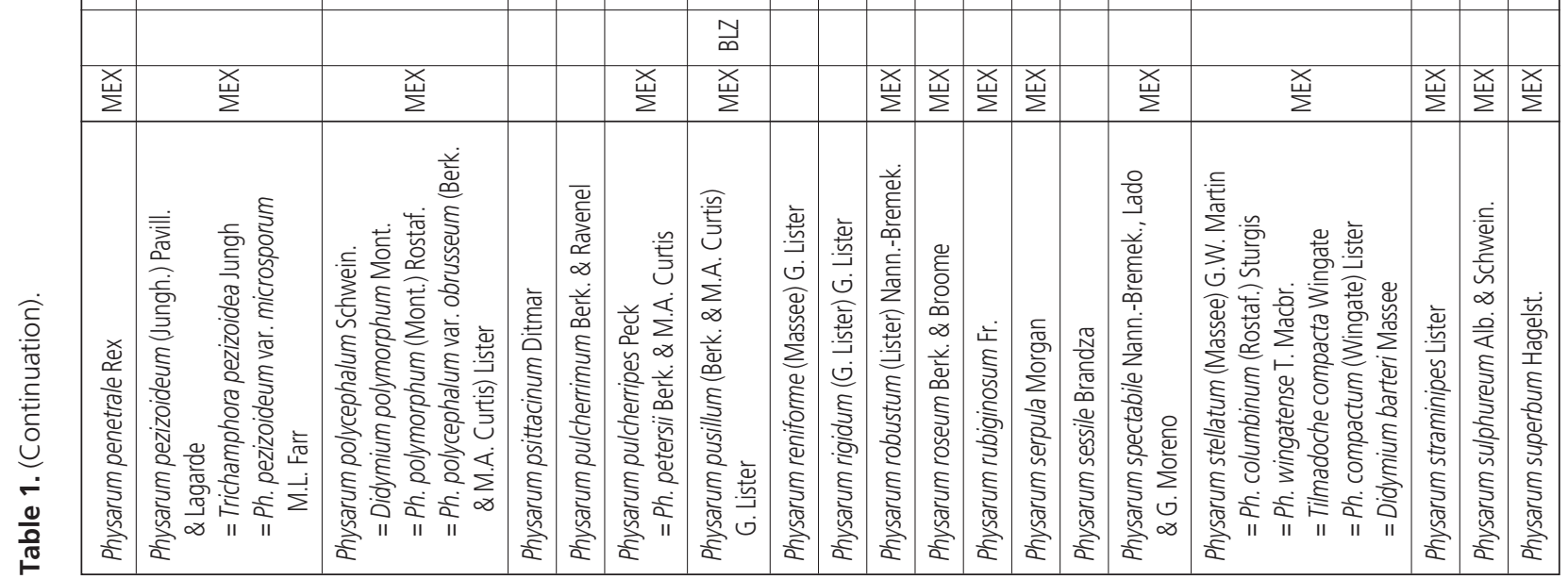




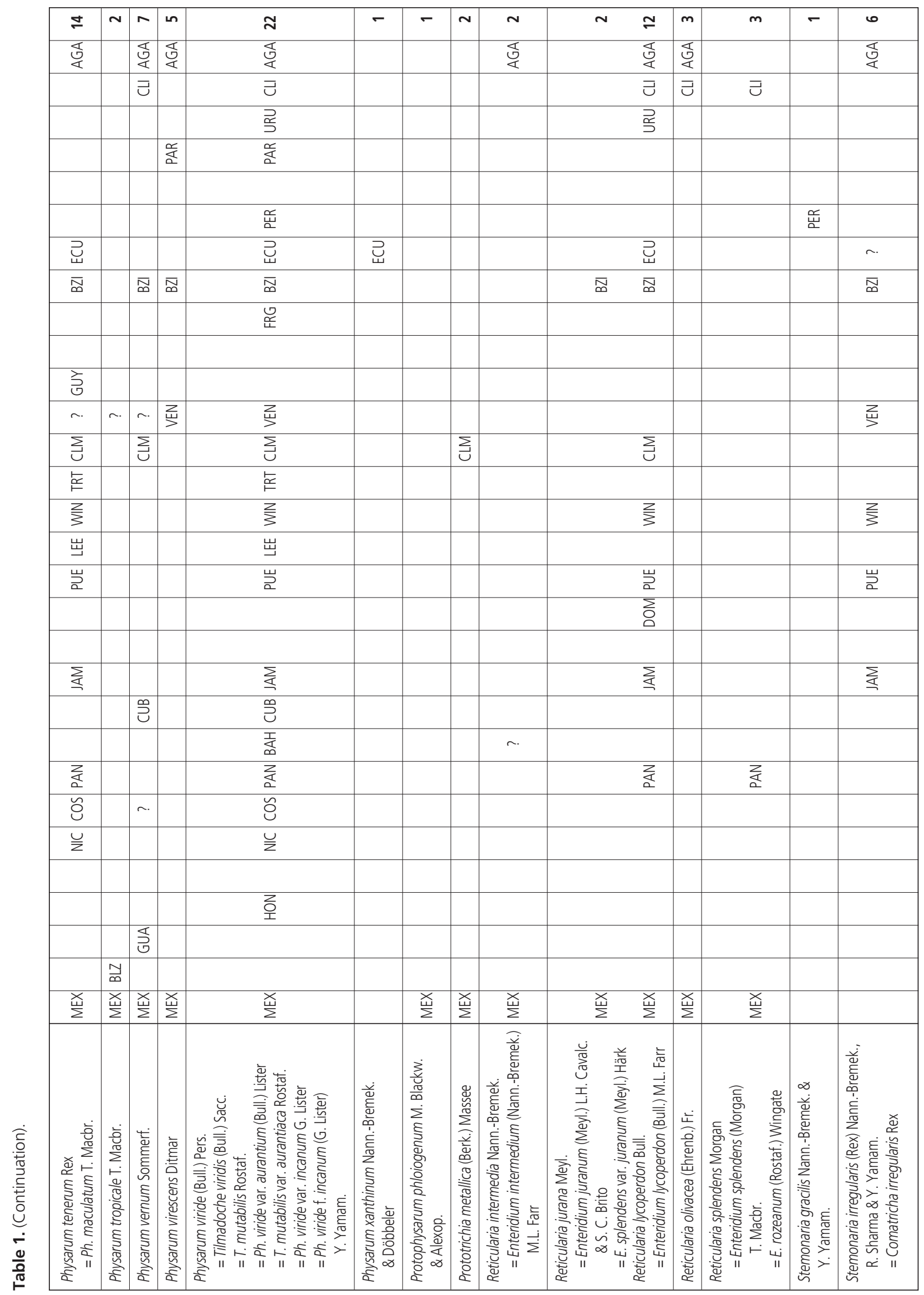




\begin{tabular}{|c|c|c|c|c|c|c|c|c|c|c|c|c|c|c|c|}
\hline$\Xi$ & $\bar{\sim}$ & $\stackrel{m}{2}$ & - & ని & $\stackrel{2}{2}$ & $\sim$ & - & $=$ & $\tilde{\sim}$ & $\nabla$ & in & $m$ & 으 & $\sim$ & $\sim$ \\
\hline 高 & 高 & 霆 & & 豪 & 芯 & 芯 & & 芯 & 正 & 玻 & & & 要 & 正 & 委 \\
\hline & $\bar{J}$ & & & こ & & & & & こ & & & & こ & & \\
\hline 롶 & $\stackrel{\vec{P}}{\mathrm{I}}$ & & & $\stackrel{\vec{P}}{\stackrel{s}{y}}$ & & & & & 록 & & & & & & \\
\hline & & & & & & & & & $\frac{⿱ \alpha}{\alpha} \frac{\alpha}{\alpha}$ & & & & & & \\
\hline$\vec{\varnothing}$ & & & & & & & & & ठे & & & & & & \\
\hline & 絗 & & 営 & 啋 & & & & & 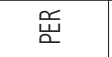 & & & & & & \\
\hline & 己 & 己. & & 己ِ & 己己 & & & 己己 & 己. & & & & & & \\
\hline న্口 & $\bar{\infty}$ & $\bar{\infty}$ & & న్థ & $\bar{\infty}$ & & న্口 & $\bar{\infty}$ & $\bar{\infty}$ & $\bar{\aleph}$ & న్థ & $\bar{\aleph}$ & হ্ল & & $\bar{\aleph}$ \\
\hline 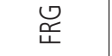 & & & & ্ֻে & & & & $\underset{\mathscr{Y}}{\mathscr{4}}$ & 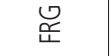 & & & & & & \\
\hline & & & & & & & & & & & & & & & \\
\hline & 恙 & 芭 & & 芭 & $\underline{\mathrm{H}}$ & & & $\underline{\mathrm{H}}$ & 慈 & & & & & & \\
\hline & $\sum_{\mathrm{U}}$ & & & 仙 & & & & & $\sum_{j}$ & & & & & & \\
\hline 哀 & 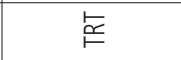 & 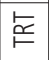 & & 店 & & & & 挋 & 占 & & & & & & \\
\hline$\sum_{3}$ & 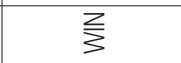 & 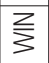 & & 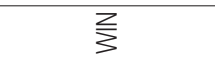 & $\varliminf_{3}$ & & & & $\varliminf_{3}$ & & 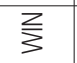 & & $\frac{z}{3}$ & & \\
\hline 岃 & 出 & & & 岁 & 岃 & & & & 岃 & & & & & & \\
\hline 嵓 & 嵓 & 岁 & & 嵓 & 嵓 & & & 嵓 & 岁 & & & & 嵓 & & \\
\hline & ¿ & & & ¿ & $\overline{0}$ & & & & & & & & & & \\
\hline & 㕵 & & & & & & & & & & & & & & \\
\hline$\sum_{\leq}$ & $\sum$ & $\sum_{\leq}$ & & $\sum_{\leq}$ & $\sum_{\leq}$ & & & $\sum_{\leq}$ & $\sum$ & & $\sum_{\leq}$ & & $\sum_{i}$ & & \\
\hline$\stackrel{9}{\mathcal{U}}$ & $\stackrel{9}{己}$ & $\stackrel{9}{\mathcal{U}}$ & & 㥯 & $\stackrel{9}{\mathcal{U}}$ & & & $\stackrel{9}{\mathrm{U}}$ & 㥯 & & & $\stackrel{9}{己}$ & & & \\
\hline & & & & & $\begin{array}{l}\frac{T}{\Delta} \\
\infty\end{array}$ & & & & & & & & & & \\
\hline$z_{a}$ & 交 & $z_{2}$ & & $\sum_{2}$ & & & & $z_{a}$ & 苔 & $z_{a}$ & 苔 & & ż & & \\
\hline & $\tilde{u}$ & $\tilde{u}$ & & $\tilde{u}$ & $\tilde{U}$ & & & & $\tilde{u}$ & & $\tilde{O}$ & & $\tilde{u}$ & & \\
\hline$\frac{u}{z}$ & $\frac{u}{z}$ & & & $\frac{u}{z}$ & & & & & $\frac{\cup}{z}$ & & & & & & \\
\hline & & & & & & & & & & & & & & & \\
\hline & 오 & & & & & & & & & & & & & & \\
\hline & & 产 & & & 言 & & & & 志 & & & & 志 & & \\
\hline & & & & & $\bar{y}$ & & & & ్ㅡ & & & & & & \\
\hline 兽 & 蔍 & 㮍 & & 离 & 齐 & 齐 & $\sim$ & 层 & 离 & 离 & & 离 & 彥 & 层 & \\
\hline 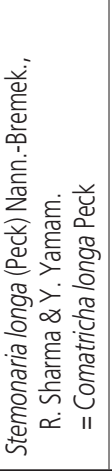 & 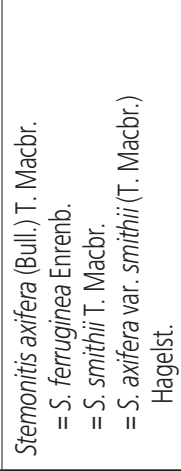 & 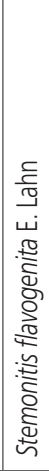 & 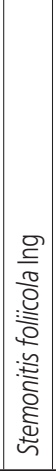 & 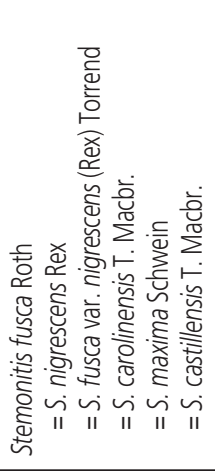 & 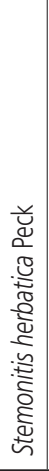 & 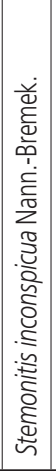 & 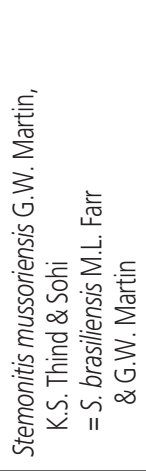 & 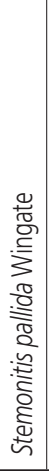 & 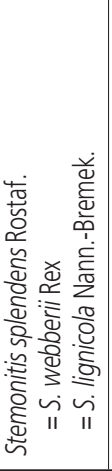 & 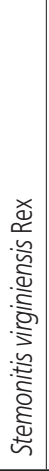 & 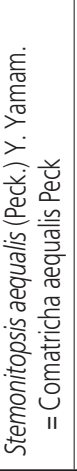 & 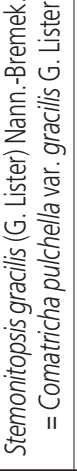 & 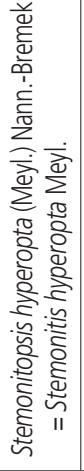 & 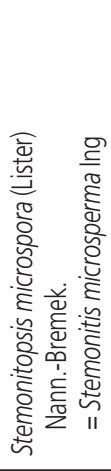 & 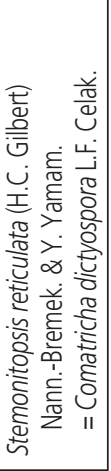 \\
\hline
\end{tabular}




\begin{tabular}{|c|c|c|c|c|c|c|c|c|c|c|c|c|c|c|}
\hline 6 & $\approx$ & - & $m$ & - & $\sim$ & - & 0 & - & - & n & - & $m$ & - & $=$ \\
\hline \multirow[t]{7}{*}{ 正 } & 志 & & 胥 & & & & & & 采 & 志 & & & 委 & 余 \\
\hline & $\bar{\nu}$ & & & & $\sim$ & & $\bar{U}$ & & & $\bar{u}$ & & $\bar{U}$ & & $\bar{u}$ \\
\hline & 록 & & & & & & & & & & & & & \\
\hline & & & & & & & & & & & & & & \\
\hline & & & & & & & & & & & & & & \\
\hline & 㩊 & & & & & & & & & & & & & \\
\hline & 겁 & & & & $\sim$. & 겁 & 己 & & & & & & & 己 \\
\hline \multirow[t]{4}{*}{$\bar{\infty}$} & $\bar{\infty}$ & & $\bar{\infty}$ & $\bar{\infty}$ & & & & & & $\bar{\infty}$ & & $\bar{\aleph}$ & & $\bar{\aleph}$ \\
\hline & ঙ্ঙ্ড & & & & & & & & & & & & & \\
\hline & & & & & & & & & & & & & & \\
\hline & ड़ & & & & & & & & & & & & & \\
\hline \multirow[t]{3}{*}{ 妾 } & 妾 & & & & 妾 & & & & & & 妾 & & & 妾 \\
\hline & $\sum_{j}$ & & & & & & & & & & & & & \\
\hline & 占 & & & & & & 点 & & & & & & & \\
\hline \multirow[t]{9}{*}{ 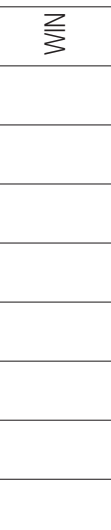 } & 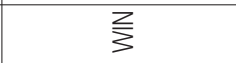 & & & & & & & & & & & & & \\
\hline & 岜 & & & & & & & & & & & & & \\
\hline & 嵓 & & & & & & & & & & & & & 容 \\
\hline & ¿ & & & & & & & & & ¿o & & & & \\
\hline & & & & & & & & & & & & & & \\
\hline & $\sum_{\leq}$ & & & & & & & & & $\sum_{1}$ & & & & $\sum_{1}$ \\
\hline & 急 & & & & & & 胥 & & & & & & & 党 \\
\hline & & & & & & & & & & & & & & \\
\hline & 交 & & & & & & 交 & & & & & & & \\
\hline \multirow[t]{5}{*}{$\tilde{\cup}$} & $\tilde{O}$ & & & & & & & & & $\tilde{O}$ & & & & $\tilde{O}$ \\
\hline & $\frac{u}{z}$ & & & & & & & & & & & & & \\
\hline & & & & & & & & & & & & & & \\
\hline & & & & & & & & & & & & & & \\
\hline & 志 & & & & & & & & & & & & & S \\
\hline 尼 & 莖 & 忞 & 嵒 & & 屆 & & 屑 & x & & 屈 & & 접 & & 学 \\
\hline 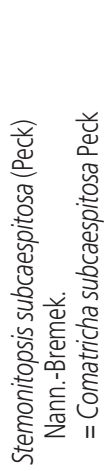 & 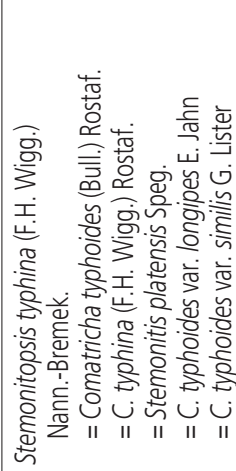 & 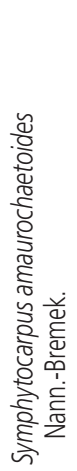 & 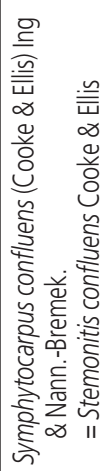 & 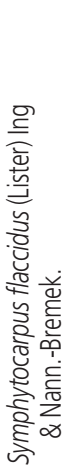 & 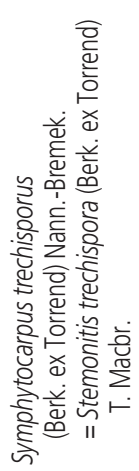 & 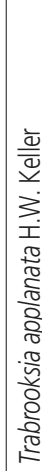 & 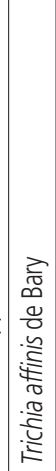 & 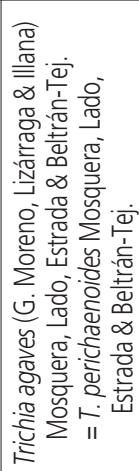 & 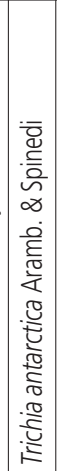 & 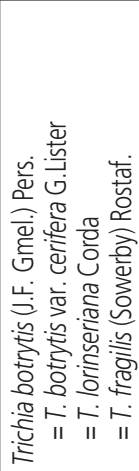 & 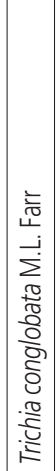 & 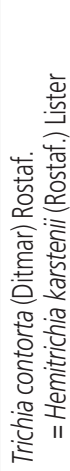 & 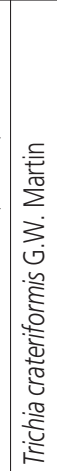 & 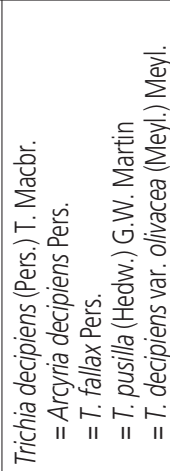 \\
\hline
\end{tabular}




\begin{tabular}{|c|c|c|c|c|c|c|c|c|c|c|c|c|c|c|c|c|c|}
\hline- & ని & $N$ & $\sim$ & - & - & $\sigma$ & $\infty$ & - & $\sigma$ & or & 0 & $\sim$ & $\overline{-}$ & $\stackrel{m}{-}$ & $\stackrel{\infty}{\simeq}$ & $\sigma$ & \\
\hline & 壳 & & & $\underset{4}{\mathbb{1}}$ & & & $\underset{4}{\mathbb{1}}$ & 蹗 & 昏 & 莛 & & $\underset{4}{\mathbb{3}}$ & & 㜽 & $\mathbb{4}$ & 㜽 & 이 \\
\hline & $\bar{U}$ & & & & 己 & $\bar{u}$ & & & $\bar{U}$ & $\bar{U}$ & & & & $\bar{U}$ & & & ฐ \\
\hline & $\stackrel{\vec{P}}{\vec{s}}$ & & & & & & & & & & & & & & 곡 & 옥 & $\tilde{ก}$ \\
\hline & & & & & & & & & $\frac{\alpha}{\alpha}$ & & & & & & & & ని \\
\hline & $\overrightarrow{\mathrm{D}}$ & & & & & & & & & & & & & & & & $₹$ \\
\hline & 䍃 & & & & & 獣 & & & & & & & & & & 営 & $\bar{m}$ \\
\hline & 己. & & & & & & 己. & & 己. & & & & & 검 & 己. & & $\stackrel{\dddot{m}}{\circ}$ \\
\hline & $\bar{\infty}$ & & $\bar{\infty}$ & & & & $\bar{\infty}$ & & & $\bar{\infty}$ & $\bar{\Phi}$ & & $\bar{\infty}$ & $\bar{\infty}$ & $\bar{\aleph}$ & $\bar{\infty}$ & సి \\
\hline & 만 & & & & & & & & & & $\underset{\Psi}{\Psi}$ & & & ত্ㄴ & 문 & & $\hat{m}$ \\
\hline & & & & & & & & & & & & & & & 答 & & a \\
\hline & & & & & & & & & & & & & & & & & $\simeq$ \\
\hline & 辛 & & & & & & 辛 & & 妾 & & 妾 & & & & 妾 & 妾 & $\Xi$ \\
\hline & $\sum$ & & & & & & $\sum$ & & & $\sum$ & & & & & $\sum_{U}$ & & ஃ \\
\hline & 占 & & & & & & & & & & & & & & 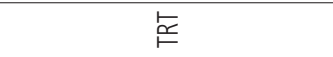 & & $\approx$ \\
\hline & 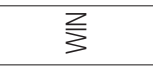 & & & & & & & & & 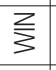 & & & & 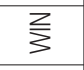 & 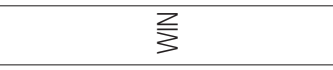 & & : \\
\hline & 岃 & & & & & & & & & & & & & 岃 & 岃 & & $\boldsymbol{\infty}$ \\
\hline & 岩 & & & & & & & & & & 嵓 & & & 嵓 & 嵓 & & m \\
\hline & ¿ & $\bar{\partial}$ & & & & & & & & & & & & ¿ & & & m \\
\hline & & & & & & & & & & & & & & & & & ని \\
\hline & $\sum_{1}$ & & & & & & $\sum_{\Sigma}$ & & $\sum$ & $\sum$ & $\sum$ & & & $\sum$ & $\sum_{\leq}$ & & $\stackrel{9}{=}$ \\
\hline & $\stackrel{9}{\mathrm{U}}$ & & & & & & & & 敛 & 敛 & & & & & $\stackrel{9}{\mathcal{U}}$ & & $\overline{-}$ \\
\hline & & & & & & & & & & & & & & & & & 으 \\
\hline & $\underset{2}{\text { 妾 }}$ & & & & & $\underset{z}{z}$ & & & & & & & & $\underset{2}{\text { 杀 }}$ & za & 杂 & 우 \\
\hline & $\tilde{u}$ & & & & & & $\tilde{O}$ & & $\tilde{u}$ & $\tilde{U}$ & $\tilde{U}$ & & & $\tilde{U}$ & $\tilde{O}$ & $\tilde{O}$ & $\mathfrak{F}$ \\
\hline & & & & & & & & & & & & & & & $\frac{U}{z}$ & & $\mathfrak{m}$ \\
\hline & & & & & & & & & & & & & & & & & 0 \\
\hline & & & & & & & & & & & & & & & & & $\simeq$ \\
\hline & & & & & & & & & & & & & & & & & $\stackrel{2}{\sim}$ \\
\hline & & & & & & & & & & & & & & & & 금 & $\bar{\sigma}$ \\
\hline 应 & 岀 & 䓂 & 离 & & & 离 & 岀 & & 离 & 离 & & 离 & & 层 & 岀 & 㞿 & $\underset{\widetilde{m}}{ }$ \\
\hline 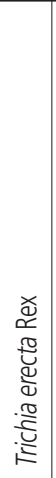 & 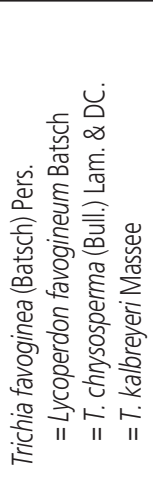 & 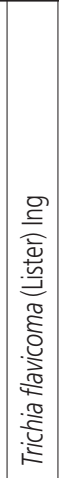 & 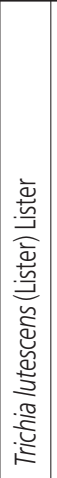 & 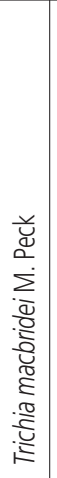 & 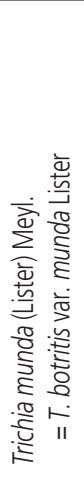 & 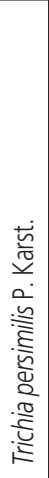 & 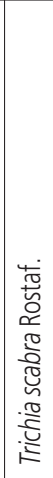 & 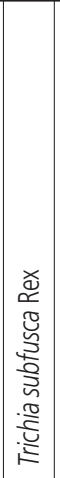 & 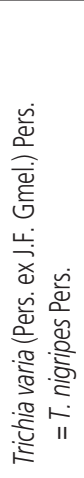 & 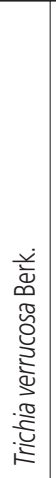 & 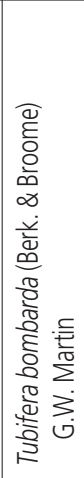 & 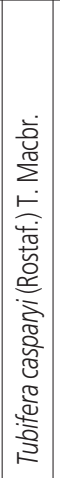 & 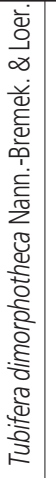 & 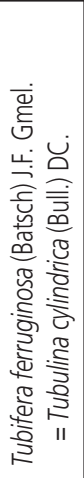 & 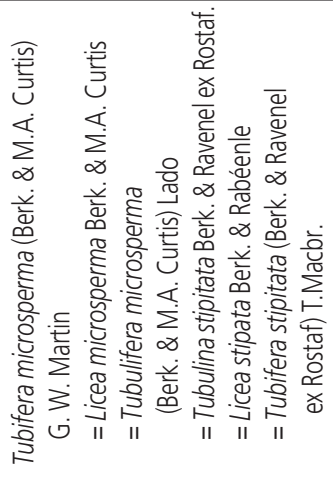 & 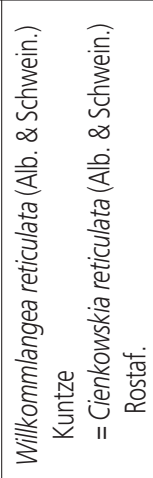 & 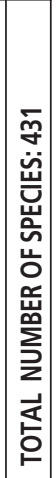 \\
\hline
\end{tabular}


Table 2. Doubtful and Excluded species.

\section{Doubtful species}

Arcyodes incarnata (Alb. \& Schwein.) O.F. Cook [= Lachnobolus incarnatus Alb. \& Schwein.] — cited by Spegazzini (1921) from Chile but Farr (1976: 280) doubted the identification. Also recorded by Lazo (1966) but probably based on Spegazzini's record.

Arcyria carletae Hertel — described from Brazil (Hertel,1954). No material available for examination (Farr, 1976: 80).

Arcyria fonsecae Hertel — described by Hertel (1954) from Brazil. No material available for examination (Farr, 1976: 81).

Arcyria occidentalis (T. Macbr.) G. Lister — reported from Brazil by Teixeira (1971), probably based on the uncertain listing by Martin \& Alexopoulos (1969), fide Farr (1976: 82).

Arcyria ramulosa (F. Rudolphi) Wigand [= Trichia ramulosa F. Rudolphi] — described by Rudolphi (1829) from Peru. According to Martin \& Alexopulos (1969) could represent a new genus.

Arcyria stellfeldii Hertel — described by Hertel (1954) from Brazil. No material available for examination (Farr, 1974: 82).

Arcyria versicolor W. Phillips - cited by Torrend (1915) from Brazil and Spegazzini (1909) and Digilio (1946) from Argentina. Highly improbable, fide Farr (1976: 83). Chondrioderma frustulosum Pat. \& Lagerh. — cited by Patouillard \& Lagerheim (1895a) from Ecuador, probably a synonym of Diderma globosum, fide Farr (1976: 205).

Comatricha fluminensis (Speg.) \& Torrend [= Stemonitis fluminensis Speg.] — described by Spegazzini (1881) from Brazil. Identity uncertain, fide Farr (1976: 279). Comatricha suksdorfii Ellis \& Everh. — cited by Torrend (1915) from Brazil. Identity uncertain, fide Farr (1976: 266).

Comatricha typhoides var. cinerea Hertel — described by Hertel (1955) from Brazil. No material available for examination (Farr, 1976: 267).

Cribraria colossea Speg. - described by Spegazzini (1909) from Argentina, probably a synonym of C. tenella Schrad., fide Farr (1976: 54).

Cribraria staminiformis Speg. — described by Spegazzini (1880) from Argentina. Identity uncertain, fide Farr (1976: 56).

Didymium discoideum Torrend — described from Brazil by Torrend (1915). No material available for study, fide Farr (1976: 235).

Didymium ossicola Pat. \& Gaillard — described by Patouillard \& Gaillard (1888) from Venezuela. Identity uncertain, fide Farr (1976: 236).

Didymium platense Speg. — described by Spegazzini (1899) from Argentina. Identity uncertain.

Didymium pruinosum Berk. \& M.A. Curtis - described in Berkeley (1868) from Cuba. Identity uncertain, fide Farr (1976: 236)

Enteridium antarcticum Speg. - described from Chile by Spegazzini (1887b) and cited from Argentina (Spegazzini, 1912), probably a synonym of Reticularia olivacea, fide Farr (1976: 40)

Hemitrichia insignis Torrend — described by Torrend (1916) from Brazil. Identity uncertain.

Hemitrichia pusilla Speg. [= Hemiarcyria pusilla (Speg.) Berl. = Arcyria pusilla (Speg.) Massee] — described by Spegazzini (1881a) from Argentina. Identity uncertain, fide Farr (1976: 101)

Lamproderma inconspicuum J. Schröt. — reported from Brazil by Hennings (1896). Identity uncertain, fide Farr (1976: 253).

Lamproderma sauteri Rostaf. — reported from Brazil by Farr $(1960,1968)$. Identity uncertain, moldy material, fide Farr $(1976: 253)$.

Licea floriformis T.N. Lakh. \& R.K. Chopra [= Licea floriformis var. aureospora M.T.M. Willemse \& Nann.-Bremek. = L. longa Flatau] - reported from Ecuador by McHugh (2005). Identity uncertain

Licea schoenleinii Johow — described by Johow (1896) from Chile. Identity uncertain, fide Farr (1976:28).

Lycogala platense Speg. - described from Argentina by Spegazzini (1899a) but identity uncertain, fide Farr (1976: 36).

Paradiacheopsis erythropodia Ing; reported from Mexico by Estrada-Torres \& al., (in press). Identity uncertain.

Physarum albescens Ellis ex T. Macbr. — reported from Brazil by Cavalcanti (2002). Identity uncertain.

Physarum chlorinum Cooke - cited by Cooke (1877) from Guyana. Identity uncertain, fide Farr (1976: 173).

Physarum conglomeratum (Fr.) Rostaf. — cited by Lister (1898a) from Antigua and Torrend (1908) from "Antilles". Identity uncertain, fide Farr (1976: 173).

Physarum anomalum (Massee) Torrend [= Tilmadoche anomala Massee] — cited by Massee (1889) from Venezuela. Identity uncertain.

Physarum crustiforme Speg. — described by Spegazzini (1899b) from Argentina. Identity uncertain, fide Farr (1976: 281).

Stemonitis confluens var. "minuta?" Batista — described by Batista (1949) from Brazil. Identity uncertain, fide Farr (1976: 279).

Stemonitis curitibensis Hertel — described by Hertel (1955) from Brazil. Identity uncertain, fide Farr (1976: 279).

Stemonitis fluminensis Speg. - described by Spegazzini (1881a) from Brazil. Identity uncertain fide Farr (1976: 279).

Trichia turbinata (Bolton) Whit. [= T. chrysosperma var. turbinata "Hds."] — cited by Berkeley (1868) from Cuba, by Spegazzini (1886) from Paraguay and by Montagne (1852a, 1852b) from Chile. Identity uncertain.

\section{Excluded species}

Chondrioderma andinum Speg. — an unpublished species, fide Farr (1976: 280), cited from Argentina.

Colloderma pustulatum G.W. Martin - the name apparently has remained unpublished, fide Farr (1976: 246), cited from Mexico.

Comatricha platensis Speg. - apparently an unpublished name, fide Farr (1976: 266), cited from Argentina.

Cornuvia minutula Speg.- described from Brazil. Identity uncertain fide Farr (1976: 280).

Licea berteroana Mont. — described from Chile. Not a myxomycete, fide Martin \& Alexopoulos (1969).

Licea guaranitica Speg. — described from Paraguay. Not a myxomycete, fide Farr (1976: 28).

Tubulina guaranitica (Speg.) Speg. - based on Licea guaranitica Speg. See comments under this species

Reticularia affinis Berk. \& M.A. Curtis - described from Cuba. Not a myxomycete, fide Martin \& Alexopoulos (1969).

Reticularia atrorufa Berk. \& M.A. Curtis - described from Cuba. Not a myxomycete, fide Farr (1976: 41).

Reticularia pyrrhospora Berk. - described from Cuba, not a myxomycete, fide Farr (1976: 41).

Reticularia venulosa Berk. \& M.A. Curtis - described from Cuba. Not a myxomycete, fide Martin \& Alexopoulos (1969).

Rostafinskia australis Speg. — described from Argentina. Not a myxomycete, fide Farr (1976: 281).

Physarum areolatum Bertero - apparently an unpublished name, fide Farr (1976: 173), cited from Chile.

Physarum argentinense Speg. — apparently and unpublished name, fide Farr (1976: 173), cited from Argentina. 


\section{Discussion}

This review includes 431 myxomycete taxa from 51 genera reported from countries of the Neotropics. This is almost half of the total number of species known in the world (Lado, 2008), and in four decades of research, nearly double the number previously published from the region by Farr (1976). It includes $86 \%$ of known genera, most $(71 \%)$ represented by more than one species. Of the thirty countries included in Table 1, Mexico, with 323 species, has by far the largest number of myxomycete species registered, and El Salvador has the least, since no myxomycetes have yet been published from this country. Although eleven countries have over 100 species published, twelve of the countries have recorded fewer than $10 \%$ of the total (Table 1). Very few species can be considered pan-neotropical as only 22 of the species $(5 \%)$ were found in 20 countries or more, whereas 144 species $(33 \%)$ have been reported from only one country in the Neotropics. Some of the latter have only been found in a single country worldwide, such as Arcyriatella congregata, Calomyxa synspora, Diderma robustum, or Physarum bubalinum, but others have been reported from other countries in different parts of the world, and still others have been recently described, and may well be found to be more widely distributed in the future.

Arcyria cinerea has been reported from 28 of the 30 countries. This species, and many of the others that make up the 5\%, such as Arcyria denudata, Cribraria cancellata, Didymium nigripes, D. squamulosum, Fuligo septica, Hemitrichia calyculata, H. serpula, Lycogala epidendrum, Perichaena chrysosperma, Physarum album, Ph. viride, Stemonitis fusca or Trichia favoginea, are the most common species in many environments, and considered to be generalists, with the ability to exploit the conditions in both temperate and tropical habitats. In addition, there seem to be ecotypes or varieties of some species in the tropics which future work may show are species complexes, but they are all included at present under the same name. The assemblage of myxomycetes which does appear to be characteristically Neotropical, in that they have been recorded from many of the strictly tropical countries, includes Ceratiomyxa morchella, C. sphaerosperma, Comatricha tenerrima, Craterium paraguayense, Cribraria microcarpa, C. tenella, Diachea bulbillosa, Diderma chondrioderma, Didymium intermedium, Lycogala conicum, L. exiguum, Pbysarella oblonga, Physarum aeneum, $\mathrm{Ph}$. crateriforme, $\mathrm{Ph}$. fulgens, $\mathrm{Ph}$. javanicum, $\mathrm{Ph}$. nicaraguense, $\mathrm{Ph}$. nucleatum, $\mathrm{Ph}$. oblatum, $\mathrm{Ph}$. roseum, Ph. stellatum, Ph. superbum, Stemonaria longa, Stemonitis herbatica, S. pallida, Tubifera bombarda and T. microsperma.
The list also includes 13 new species that have been described in the last decade from material from the Neotropics, which are Calonema foliicola Estrada, J.M. Ramirez \& Lado, Cribraria fragilis Lado \& Estrada, C. zonatispora Lado, Mosquera \& Beltrán-Tej., Diderma acanthosporum Estrada \& Lado, D. yucatanensis Estrada, Lado \& S.L. Stephenson, Didymium tehuacanense Estrada, D. Wrigley \& Lado, D. umbilicatum D. Wrigley, Lado \& Estrada, D. wildpretii Mosquera, Estrada, Beltrán-Tej., D. Wrigley \& Lado, Licea succulenticola Mosquera, Lado, Estrada \& Beltrán-Tej., Macbrideola berrerae Lizárraga, G. Moreno \& Illana, M. lamprodermoides G. Moreno, Lizárraga \& Illana, Perichaena stipitata Lado, Estrada \& D. Wrigley, and Trichia agaves (G. Moreno, Lizárraga \& Illana) Mosquera, Lado, Estrada \& Beltrán-Tej. (described as Hemitrichia agaves).

The most representative order from the Neotropics was the Physarales, which is also the order with the greatest number of species. However, if the size of each order is looked at as a percentage of the total number of myxomycete species (Fig. 3) and compared to the percentage of each order found in the Neotropics, it can be seen that the Physarales are indeed more prevalent and more diverse in this area. Among these were 75 species of Physarum and 42 species of Didymium, the most representative genera. Almost $50 \%$ of the species in arid areas of Chile or Mexico (Lado \& al, 2007, Estrada \& al, in press) and over $40 \%$ in a cloud forest in Ecuador (Schnittler \& al., 2002) were also from this order. Not all tropical areas are the same, however, since very few species of these genera were found in the high elevation forests of Costa Rica (Rojas \& Stephenson, 2007). The orders Trichiales and Echinosteliales also seem to be better represented in the Neotropics than are the Stemonitales or Liceales (Fig. 3).

Myxomycetes have been found to date in all major biomes (Ing, 1994), living on decaying remains of all types of vegetation. Information on vegetation, and particularly vascular plants, as the habitats and substrates for all myxomycetes, is therefore fundamental to understanding their distribution. In the Neotropical region, even specific parts of plants have been shown to be new microhabitats for myxomycetes, such as the inflorescences of tropical plants (Schnittler \& Stephenson, 2002a, 2002b), living and dead lianas (Wrigley de Basanta \& al., 2008), or the interior of succulent plants (Estrada-Torres \& al., in press). Other potential microhabitats probably exist too in poorly studied vegetation, such as the mangrove swamps or the grasslands of the pampa. In addition to information on the vegetation, other factors must be taken into consideration when analyzing the data compiled above in Table 1 . The geographical exten- 


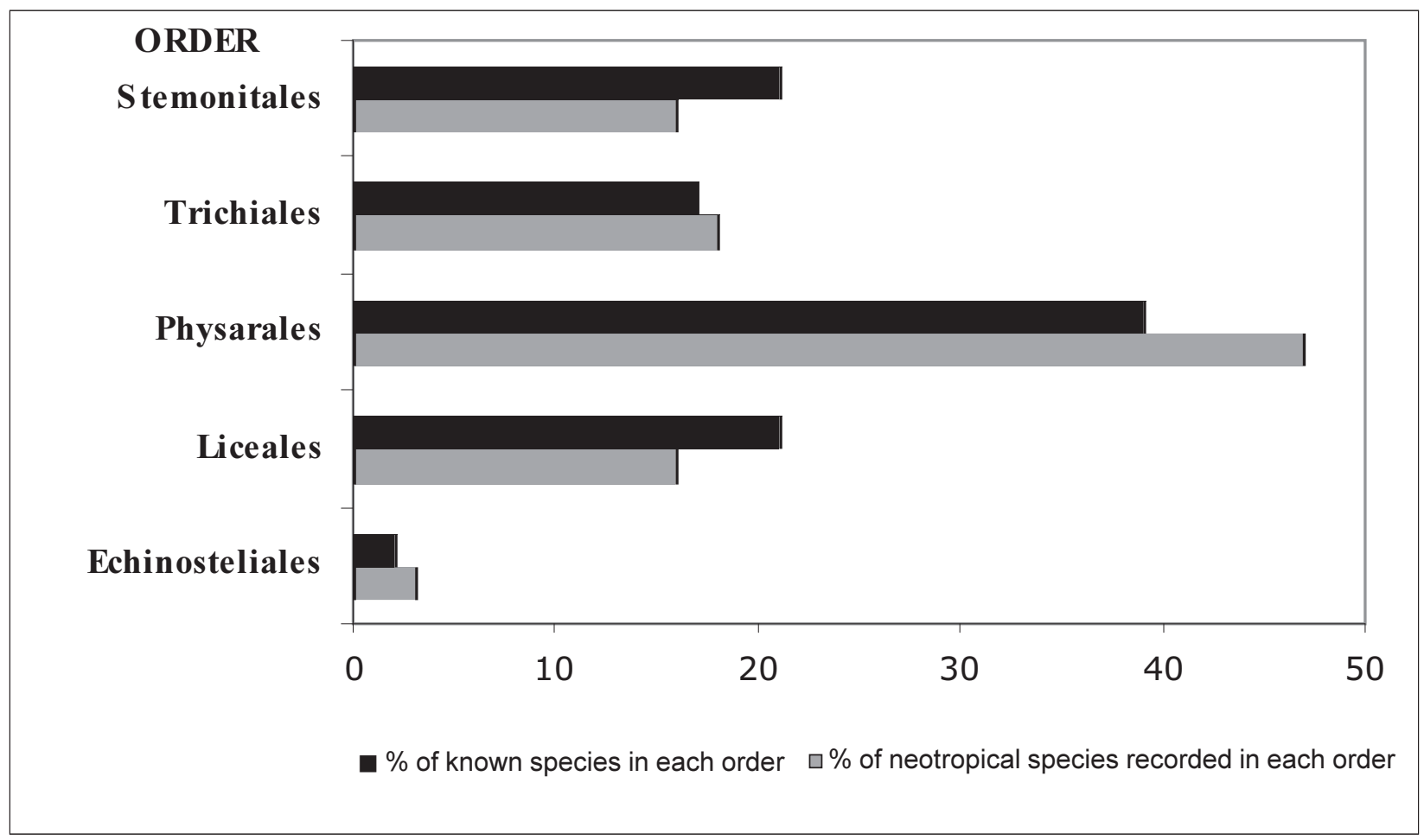

Fig. 3. Percentage of known Myxomycetes in different Orders compared to the percentage of species from the Neotropics in each order.

sion of each country obviously affects the potential number of myxomycetes to be found, and also the extent to which the country has been studied. The problem with any biogeographical analyses, as mentioned by Morawetz \& Raedig (2007), is the difficulty in measuring the sampling effort. As the authors note, there can be insufficient sampling in places which are difficult to access, and intense sampling in easily accessible places. In order to enable a more realistic evaluation of the data in Table 1 to be made, a comparison of some of this information is given in Table 3 .

Mexico, with the greatest number of myxomycetes, including all thirteen of the new species described from the region in the last decade, has been surveyed in a number of recent projects. Records appear in 138 papers, of which almost 100 are since 1990 (Table 3), but its myxomycete richness is also because it is so geographically diverse. It is the area where Boreal, Neotropical and Caribbean vegetation overlap. Some of these areas do not strictly belong to the Neotropics, as mentioned earlier, but for the purposes of this paper the records from all parts of the countries like Mexico and Argentina have been included, since records of some surveys did not include specific locations. Some parts of Mexico are in Central America, but others pertain to North America. For this reason we treat it alone. Mexico is also a country of varied relief with the trans-Mexican volcanic belt perpendicular to the two north-south mountain chains. This creates a profusion of different microclimates and vegetation islands in the valleys and consequently many varied habitats for myxomycetes. Its diversity of vegetation according to Davis \& al. (1997) is comparable only to India and Peru. Rzedowski (1991) also highlights the high level of plant endemism particularly in arid or semi-arid areas and subhumid montane highlands. Recent surveys have been done in arid areas of the country, such as the Tehuacán-Cuicatlán Valley in Puebla and Oaxaca (Estrada-Torres \& al., in press) or Sonora (Lizárraga \& al., 2007, 2008), in tropical moist forests such as El Eden in Quintana Roo, and Los Tuxtlas in Veracruz (Lado \& al., 2003) or in a dry forest in Chamela, Jalisco (Lado \& al., 1999), among others. Many areas of interest remain to be studied in Mexico, however, including the Lacandona region (Chiapas), the Upper Mezquital River region (Durango), or the Sierra de Juárez (Oaxaca).

Central America is of particular interest as it is the land bridge between North and South America, literally closing the circulation between the Pacific and the Atlantic oceans during its formation, and permitting the exchange of organisms between the two land masses. The region has a very varied topography, with 
Table 3. Neotropical countries included in this paper: A comparison of land area, vascular plant species richness, myxomycete species richness and number of publications; na = information not available. Sources: Gentry (1982, 1992); Davis \& al. (1997).

\begin{tabular}{|c|c|c|c|c|}
\hline Country & Area $\left(\mathrm{km}^{2}\right)$ & Vascular plants (approx.) & Myxomycete species & Publications \\
\hline Mexico & $1,972,546$ & 30,000 & 323 & 138 \\
\hline \multicolumn{5}{|l|}{ Central America } \\
\hline Belize & 22,800 & 4,400 & 41 & 1 \\
\hline Costa Rica & 51,060 & 10,500 & 143 & 16 \\
\hline El Salvador & 20,720 & 2,500 & 0 & 0 \\
\hline Guatemala & 108,430 & 8,000 & 26 & 2 \\
\hline Honduras & 111,890 & 6,000 & 12 & 4 \\
\hline Nicaragua & 118,750 & 7,000 & 33 & 5 \\
\hline Panama & 75,990 & 9,000 & 106 & 14 \\
\hline \multicolumn{5}{|l|}{ Caribbean } \\
\hline Bahamas & 14,260 & 1,300 & 10 & 1 \\
\hline Cuba & 108,722 & 7,000 & 101 & 25 \\
\hline Dominican Republic & 48,441 & 5,500 & 39 & 8 \\
\hline Haiti & 27,749 & with Dominican Republic & 20 & 3 \\
\hline Jamaica & 11,425 & 3,700 & 119 & 10 \\
\hline Leeward Islands & na & na & 83 & 12 \\
\hline Puerto Rico & 8,897 & 2,900 & 93 & 19 \\
\hline Trinidad and Tobago & 4,838 & 2,600 & 62 & 7 \\
\hline Windward Islands & na & na & 106 & 12 \\
\hline \multicolumn{5}{|l|}{ South America } \\
\hline Argentina & $2,736,690$ & 9,370 & 160 & 46 \\
\hline Bolivia & $1,084,380$ & 17,350 & 42 & 5 \\
\hline Brazil & $8,456,510$ & 56,000 & 206 & 114 \\
\hline Chile & 748,800 & 5,200 & 102 & 19 \\
\hline Colombia & $1,038,700$ & 45,000 & 96 & 12 \\
\hline Ecuador & 276,840 & 21,000 & 136 & 31 \\
\hline French Guiana & 90,976 & 4,000 & 37 & 2 \\
\hline Guyana & 196,850 & 6,400 & 12 & 7 \\
\hline Paraguay & 397,300 & 8,000 & 20 & 9 \\
\hline Peru & $1,280,000$ & 19,000 & 31 & 5 \\
\hline Surinam & 156,000 & 5,000 & 9 & 4 \\
\hline Uruguay & 174,810 & 2,270 & 52 & 8 \\
\hline Venezuela & 882,050 & 21,070 & 111 & 19 \\
\hline
\end{tabular}

mountains, valleys and high plateaus, which affects the climate and vegetation types. Although it is made up of small countries, it contains up to $8 \%$ of the world's plant species (Davis \& al., 1997) many of which are endemic (Gentry, 1992). Some of the Central American countries have been sampled in several surveys recently, most notably Costa Rica (Schnittler \& Stephenson, 2000) and Panama (Pando, 1997) which have more than 100 species of myxomycete recorded, but others such as El Salvador have not been studied at all. The three largest countries Guatemala, Honduras and Nicaragua are only cited in a total of 11 publications and have fewer than 50 myxomycete species recorded between them of the 195 different species recorded from Central America, apart from Mexico (Table 3). Even in well studied countries there are vegetation types or specific areas which have received little or no attention. For instance in Costa Rica (16 publications), a recent study (Rojas \& Stephenson, 2007) in high elevation oak for- 
est apported 11 new species to the country record, as previously there had been very little work done in that type of forest. Of the great variety of vegetation in Central America from lowland rain forest and swamps to arid areas full of cacti, the Petén region of Guatemala or the Darién Province of Panama are unexplored endangered areas and of great interest for future work.

The Caribbean Islands are a group of islands with different origins, and some are remains of continents, others of recent volcanoes. Each major tropical island cluster has independently evolved its own native flora, influenced by the fact that some of the islands, such as Hispaniola, have been formed from several territories, which separated and rejoined various times in their geologic history (Davis \& al., 1997). Myxomycetes from the Caribbean have been relatively well studied (Table 3 ) and cited in 98 publications, $26 \%$ of them from Cuba, the largest island with the greatest number of vascular plant species. In addition, other islands, such as Jamaica or the Windwards which have been the site of intensive surveys, have as many or more myxomycetes, although reported in fewer papers. A total of 174 different myxomycete species have been recorded from these islands. Some areas of high plant diversity as yet understudied for myxomycetes in these islands, are the Oriente in Cuba, the Morne Trois Pitons National Park in Dominica, the Central Highlands and Sierra de Neiba in the Dominican Republic, the Pic Macaya or the Morne La Visite in Haiti, the Blue and John Crow Mountains, or the Cockpit Country in Jamaica and the Aripo Savannas Scientific Reserve in Trinidad.

The continent of South America has about oneeighth of the Earth's land surface. It has been an island continent during most of the period of angiosperm evolution, and it has been joined to North America by the Isthmus of Panama, and then separated, more than once in its geologic history. The topography of South America is varied and ranges from the Brazilian lowland with its tropical rain forest to the snowcovered Andes. The Andes is the longest mountain range in the world, and stretches for over $7,000 \mathrm{~km}$ forming the backbone of the continent from Colombia to Tierra del Fuego. It has the highest mountain in the Western Hemisphere, the Aconcagua. Most of the continent of South America is in the tropics, but the elevation of the Andes and the presence of cold ocean currents, like the Humboldt stream, cause cool temperatures even at the equator. The combined effect of these environmental factors accounts for the variety of vegetation in this area, which in turn provides multiple substrates, and distinct macro and microhabitats for myxomycetes. In South America, Brazil with the largest land area (Table 3 ) has by far the largest number of vascular plants. It has also been intensively studied for myxomycetes (114 publications), but mainly in the northeastern area of the country. Vast areas of the Amazon have never been surveyed, and geographic areas of special scientific interest such as the Pantepui region, the Gran Chaco, the Transverse Dry Belt, have not been sampled. Some areas of distinct vegetation are also of great interest such as the Atlantic moist forest, the Tabuleiro forest, the Caatinga of north-eastern Brazil, or the Cerrado of central Brazil.

Argentina has the second largest land mass and the third number of myxomycete species of the region to date. It was initially studied for these organisms over a hundred years ago, but until recently little intensive work has been done since (only 46 publications). A recent research project, Myxotropic, was undertaken to study the Myxomycetes that develop on endemic succulent plants (Cactacea and sclerophyllous shrubs) of the Neotropical region. The first phase of the project was directed towards the study of this group of organisms from arid regions of Mexico (Estrada Torres \& al., in press). The second phase of the Project, involves the unexplored arid areas of Argentina and the North of Chile, specifically the desert areas of El Monte and Atacama, which are among the most arid of the planet (Lado \& al., 2007a). This study extends and complements a current International project "Global Biodiversity of Eumycetozoa" supported by the National Science Foundation (NSF) of the United States, developed to investigate unexplored regions of the world (Stephenson \& al., 2005). To date the Myxotropic project has provided more than 1200 myxomycete collections from Argentina which are currently under study.

The number of different myxomycete species recorded from all of South America is 328. It is evident from the list that several countries are totally undersurveyed. For example, considering their landmass and richness in vascular plants, Peru, Colombia, and Bolivia have relatively few myxomycete records. Some of the specific areas of interest in these countries would be the Iquitos region, or the eastern slopes of the Andes in Peru, the Chocó or the ChiribiqueteAraracuara-Cahuinarí region in Colombia, and the Gran Chaco or the Madidi-Apolo region of Bolivia. There are a number of ecosystems and types of vegetation, that span several countries on the continent, and which would provide interesting data and analyses on the role of specific microhabitats or plant species in the distribution of myxomycetes. Some of these are mangrove swamps, dry forest, the cerrado and caatinga, open grass savanna, the Patagonian steppe, and the Valdivian forest. 
If the number of publications is taken as an indication of the amount of research done in a country, the suggestion that apparent distribution of myxomycetes follows the distribution pattern of research efforts (Stephenson \& Stempen, 1994) is supported by these data. The research intensity, however, is not always related to the number of papers since some papers listed may have only a few records while others, such as the Bahamas or Belize, may have the total for the country in one paper. Myxomycete records have appeared in fewer than ten publications from over half (16) of the 30 countries listed (Table 3 ), which gives an idea of the enormous amount of research still to be done.

This paper indicates that almost half the known species of myxomycetes have been recorded from the Neotropics. It also indicates that there are many areas that remain under studied, or not investigated at all. This can be seen graphically on the map (Fig. 4), generated by the GBIF (Global Biodiversity Information Facility) network, from their database of geo-referenced records of myxomycetes in major herbaria, which shows sparse points in many of the areas of the Neotropics and none in vast areas of the South American continent. The biodiversity occurrence data for the area mapped from this database, is provided by: University of Arkansas (2008), Staatliche Naturwissenschaftliche Sammlungen Bayerns (2008), GBIFSweden, Gothenburg Herbarium (2008), Real Jardín Botánico, Madrid (2008), Utah State University (2008).

The importance and urgency attached to the completion of more biodiversity surveys for these microorganisms in the Neotropics lies in the fact that this area contains some of the most threatened environments on earth. Habitat loss and destruction of the vegetation, to which the myxomycetes are so intimately linked, is on the increase. As Mittermeier \& al., (2004) have pointed out, the tropical Andes is the richest and most diverse biodiversity hotspot on earth, and yet only $25 \%$ of the original primary vegetation of the tropical Andes remained when Myers $\&$ al., (2000) went to press. Approximately 100 Neotropical plant narrowendemisms per year are now being lost due to forest conversion (Morawetz \& Raedig, 2007). The importance of the conservation of these habitats is not limited to the larger flora or fauna of major conservation efforts. Microorganisms, such as the myxomycetes, with unknown ecological importance and unsuspected species richness are subject to the same, or greater, risks. Protection of their gene pools, and investigation into their ecological importance, before their threatened habitats shrink further, should give high priority to biodiversity research on myxomycetes in the Neotropics.

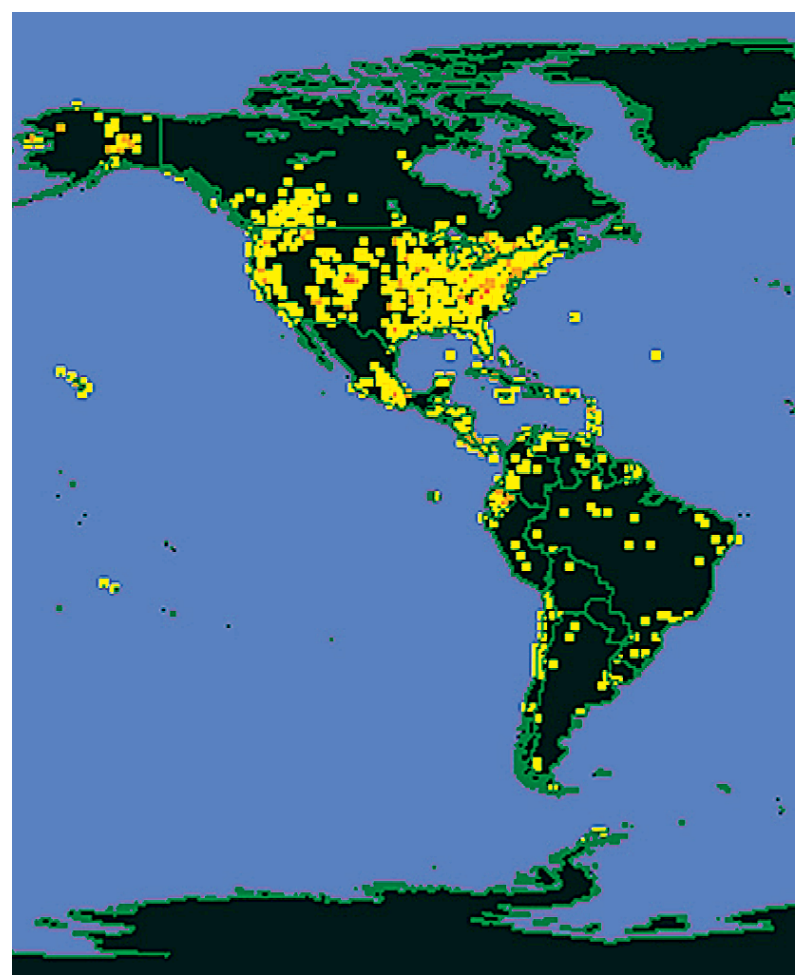

Fig. 4. Myxomycete biodiversity occurrence data from North and South America. This map only shows records with coordinates from the GBIF network and may not properly represent the total distribution of Myxomycetes.

\section{Acknowledgements}

This research has been supported by the Ministry of Education and Science of Spain [grant CGL2005-00320/BOS]. The authors thank María Aguilar, Eva García and Juan Carlos Hernández for their valuable assistance in the bibliographic search. We would also like to thank Steven L. Stephenson and the two US National Science Foundation projects [grants DEB-9705464 and DEB0316284], which facilitated some of the research in the Neotropics.

\section{References}

Alexopoulos, C.J. 1967. Taxonomic studies in the Myxomycetes I. The genus Macbrideola. Mycologia 59(1): 103-115.

Alexopoulos, C.J. 1970. Rainforest Myxomycetes. In: Odum, H.T. (ed.), A Tropical Rain Forest. U. S. Atomic Energy Commission 3:21-23.

Alexopoulos, C.J. \& Beneke, E.S. 1954a. A new species of Comatricha from Jamaica. Mycologia 46: 245-247.

Alexopoulos, C.J. \& Beneke, E.S. 1954b. Myxomycetes from Jamaica. Transactions British Mycological Society 37(3): 306-313.

Alexopoulos, C.J. \& Blackwell, M. 1968. Taxonomic studies in the Myxomycetes II. Physarina. Journal Elisha Mitchell Science Society 84: 48-51.

Alexopoulos, C.J. \& Sáenz, J.R. 1975. The Myxomycetes of Costa Rica. Mycotaxon 2: 223-271.

Alves, M.H \& Cavalcanti, L.H. 1996. Myxomycetes em palmeiras (Arecaceae). Acta Botanica Brasílica 10: 1-7. 
Andrade-Torres, A. 1998. Riqueza, abundancia y diversidad de myxomycetes sobre hojarasca, troncos caidos y cortezas de árbolestropicales vivos. www.uv.mx/CITRO/el_eden/research /papers/andrade_torres/

Andrade-Torres, A., Meza-Hernández, E.A. \& Cuevas-Suárez, C. 2002a. Los Myxomycetes del estado de Quintana Roo, México. In: Guzmán, G. \& Mata, G. (eds.), Estudios sobre los hongos latinoamericanos. Resúmen IV Congreso Latinoamericano de Micología: 252.

Andrade-Torres, A., Meza-Hernández, E.A. \& Cuevas-Suárez, C. 2002b. Los Myxomycetes del parque ecológico El Haya, Xalapa, Veracruz, México. In: Guzmán, G. \& Mata, G. (eds.), Estudios sobre los hongos latinoamericanos. Resúmen IV Congreso Latinoamericano de Micología: 253.

Arambarri, A.M. 1972. Una nueva especie de Myxomycetes de Tierra del Fuego (Argentina). Boletín de la Sociedad Argentina de Botánica 14: 154-156.

Arambarri, A.M. 1973. Myxomycetes de Tierra del Fuego I. Especies nuevas y críticas del género Diderma (Didymiaceae). Boletín de la Sociedad Argentina de Botánica 15: 175-182.

Arambarri, A.M. 1975. División Myxophyta, Clase Myxomycetes. In: Guarrera \& al. (eds.), Flora criptogámica de Tierra del Fuego. Vol. 2. Fundación para la Educación, la Ciencia y la Cultura. Buenos Aires.

Baker, R.E.D. \& Dale, W.T. 1951. Fungi of Trinidad and Tobago. Commonwealth Mycological Institute Miscelaneous Publication 33: 1-123

Barbosa, M.R.V. 1996. Pesquisa Botânica Nordestina: progresso e perspectivas. Recife. Sociedade Botânica do Brasil, pp. 37-45.

Barnes, R.F. 1963. Myxomycetes from Trinidad. Transactions British Mycological Society 46(3): 453-458.

Batista, A.C. 1949. Três mixomicetes comuns em Pernambuco. Boletim Secretaria de Agricultura Indústria Comércio Estado de Pernambuco 16: 166-167.

Benjamin, C.R. \& Slott, A. 1969. Fungi of Haiti. Sydowia, Anales Mycologici Ser. II 23: 125-163.

Berkeley, M.J. 1868. On a Collection of Fungi from Cuba. Part.II., including those belonging to the Families Gasteromycetes, Coniomycetes, Hyphomycetes, Physomycetes, and Ascomycetes. Journal Linnean Society, Botany 10:341-392.

Berkeley, M.J. \& Cooke, M.C. 1876. The fungi of Brasil. Journal Linnean Society, Botany 15(86): 363-398

Berlese, A.N. 1888. Myxomyceteae Wallr. In: Saccardo, P.A., Sylloge fungorum. Ed. Sumptibus auctoris typis Seminarii 7: 323-453.

Bertero, C. 1828. Variedades Ciencias Naturales. El Mercurio Chileno 4: 194-195.

Bezerra, M.F.A., Lado, C. \& Cavalcanti, L.H. 2007. Mixobiota do Parque Nacional Serra de Itabaiana, SE, Brasil: Liceales. Acta Botanica Brasilica 21(1): 107-118.

Biodiversity occurrence data provided by: National Botanic Garden Belgium. National Museum of Nature and Science, Japan. Botanische Staatssammlung Munchen. V. L. Komarov Botanical Institute, St. Petersburg. The University of Arkansas, USA, and The Royal Botanic Garden, Madrid. (Accessed through GBIF Data Portal, www.gbif.net, 2008-05-20).

Bonar, L. 1939. Fungi from the Galapagos and other Pacific coastal islands. IV. Procceeding California Academy Science 22: 195-206.

Bononi, V.L.R., Trufem, S.F.B. \& Grandi, R.A.P. 1981. Fungos macroscópicos do Parque Estadual das Fontes do Ipiranga, Sao Paulo, Brasil, depositados no herbario do Instituto de Botânica. Rickia 9: 37-53.
Braun, K.L. \& Keller, H.W. 1976. Myxomycetes of Mexico I. Mycotaxon 3: 297-317.

Braun, K.L. \& Keller, H.W. 1986. Myxomycetes of Mexico III. Revista Mexicana de Micología 2: 25-39.

Bresadola, J. 1896. Fungi brasiliensis lecti a cl. Dr. Alfredo Möller. Hedwigia 35(5): 276-302.

Britton, N.L. \& Millspaugh, C.F. 1920. The Bahama flora. New York.

Brummitt, R.K. 2001. World Geographical Scheme for Recording Plant Distributions. $2^{\text {nd }}$ ed. TDWG. Hunt Institute for Botanical Documentation. Carnegie Mellon University, Pittsburgh.

Buyck, B. 1984. Diderma maculatum Buyck, a new Myxomycete species from Venezuela. Bulletin Jardin Botanique National Belgique 54: 131-136.

Camino, M. 1991. Myxomycetes de Cuba. I. Revista Jardín Botánico Nacional 12: 127-131.

Camino, M. 1996. Contribución al estudio de los Myxomycetes en Cuba. In: Lado, C. \& Hernández, J.C. (eds.), Abstract Volume. Second International Congress on the Systematics and Ecology of Myxomycetes: 129.

Camino, M. 1998a. Myxomycetes de Cuba. II. Orden Stemonitales. Revista Jardín Botánico Nacional 19: 147-152.

Camino, M. 1998b. Los Myxomycetes del Hoyo de Bonet, Sierra de Cubitas, Camagüey. Revista Jardín Botánico Nacional 19: 161-162.

Camino, M. \& Eliasson, U. 2002. Biodiversity of Myxomycetes in the Ecological Reserve "Alturas de Banao", Sancti Spíritus, Cuba. Scripta Botanica Belgica 22: 10.

Camino, M. \& Moreno, G. 2002. The family Stemonitaceae in Cuba: current state of studies. Scripta Botanica Belgica 22: 11.

Camino, M. \& Pérez, J.M. 2000. El género Arcyria Wiggers (Trichiales, Myxomycetes) en Cuba. Revista Jardín Botánico Nacional 21: 115-126.

Camino, M. \& Pérez, J.M. 2001. Los Myxomycetes de la Reserva Ecológica "Alturas de Banao" (El Naranjal), Sancti Spíritus. Revista Jardín Botánico Nacional 22: 109-117.

Camino, C. \& Rodríguez, M. 2002. Nuevos registros de la familia Stemonitaceae (Myxomycetes) para Cuba: Lamproderma scintillans \& Stemonitis smithii. In: Guzmán, G. \& Mata, G. (eds.), Estudios sobre los hongos latinoamericanos. Resumen IV Congreso Latinoamericano de Micología: 251.

Camino, M., Moreno, G. \& Castillo, A. 2005. Taxonomic revision of the myxomycetes from Cuba deposited in the Farlow Herbarium (USA). Mycotaxon 93: 379-400.

Camino, M., Moreno, G. \& Castillo, A. 2007. Taxonomic revision of the myxomycetes from Cuba deposited in three reference collections: U.S. National Fungus Collections (BPI-USA), British Museum (BM-UK) and Kew (K-UK). Mycotaxon 100: 349-356.

Camino, M., Stephenson, S.L., Krivomaz, T., Wrigley de Basanta, D., Lado, C. \& Estrada-Torres, A. (in press). Biodiversity survey for myxomycetes in the mountains of central Cuba. Revista Mexicana de Micología.

Capelari, M. \& Mazeiro, R. 1988. Fungos macroscópicos do Estado de Rondônia, região dos rios Jaru e Ji-Paraná. Hoenea 15: 28-36.

Capello-García, S. \& Hernández-Trejo, H. 1990. Lista preliminar de los hongos (Macromicetos) y Myxomycetes de Tabasco, México. Universidad y Ciencia 7(13): 15-21.

Castillo, A., Illana, C. \& Moreno, G. 1996. Badhamia melanospora Speg. A species wrongly interpreted. Mycotaxon 57: 163-170.

Cavalcanti, L.H. 1970. Coleçao de Mixomicetos do Museo Paraense Emílio Goeldi. Boletim Museo Paraense Historia Natural 35: $1-5$. 
Cavalcanti, L.H. 1974a. O gênero Perichaena Fries em Pernambuco. Rickia 6: 98-117.

Cavalcanti, L.H. 1974b. Mixomicetos novos para Pernambuco. Memorias Instituto de Biociências Universidade Federal de Pernambuco 1:315-327.

Cavalcanti, L.H. 1976. Mixomicetos novos para Pernambuco II. Memorias Instituto de Biociências Universidade Federal de Pernambuco, Botânica 4: 1-19.

Cavalcanti, L.H. 1977. Mixomicetos do Cerrado II - Análise comparativa das espécies encontradas no Cerrado protegido e no queimado anualmente. Anais XXIII Congresso Nacional de Botânica, Belo Horizonte, 1977, pp. 129-140.

Cavalcanti, L.H. 1985. Mixomicetos do Cerrado III - Revisão da literatura. Anais XXXIII Congresso Nacional de Botânica, Maceió, 1982, pp. 173-179.

Cavalcanti, L.H. 1996a. Myxomycetes. In: Sampaio, E.V.S.B., Mayo, S.J. \& Barbosa, M.R. (eds.), Pesquisa Botânica no BrasilProgressos e pespectivas. pp. 35-47.

Cavalcanti, L.H. 1996b. Conocimiento actual de Myxomycetes en Brasil. Abstracts Second International Congress on the Systematics and Ecology of Myxomycetes. Madrid, pp. 82.

Cavalcanti, L.H. 2002. Biodiversidade e distribuição de mixomicetos em ambientes naturais e antropogênicos no Brasil: espécies ocorrentes nas Regiões Norte e Nordeste. In: Araújo, E.L., Moura, A.N., Sampaio, E.V.S.B., Gestinari, L.M.S. \& Carneiro, J.M.T. (eds.), Biodiversidade, conservação e uso sustentável da flora do Brasil, pp. 209-216. Universidade Federal Rural de Pernambuco, Sociedade Botânica do Brasil, Recife.

Cavalcanti, L.H. \& Araújo, V.L.F. 1985. Myxomycetes da Paraíba II. Liceales. Anais VIII Reunião Nordestina de Botânica, 1984, pp.193-198.

Cavalcanti, L.H. \& Brito Jr., S.C. 1990. Enteridiaceae do Brasil. Biologia Brasilica 2(2): 115-134.

Cavalcanti, L.H. \& Dias Filha, M.C.C. 1985. Myxomycetes sobre briófitas. Anais VIII Reunião Nordestina de Botânica, 1984, pp. 223-228.

Cavalcanti, L.H. \& Fortes, S.T. 1994. Myxomycetes de Florianópolis (Santa Catarina - Brasil). Acta Botanica Brasilica 8(1): 65-75.

Cavalcanti, L.H. \& Fortes, S.T. 1995. Myxomycetes do Estado de Santa Catarina, Brasil. Boletim Sociedade Broteriana, Sér. 2, 67: $23-35$

Cavalcanti, L.H. \& Marinho, M.G.V. 1985. Myxomycetes da Paraíba I Trichiales. Anais VIII Reunião Nordestina de Botânica, 1984. Recife, pp. 185-191.

Cavalcanti, L.H. \& Mobin, M. 2001. Hemitrichia serpula var. piauiensis (Trichiaceae-Myxomycetes) A new variety from Brazil. Acta Botanica Brasilica 15: 133-137.

Cavalcanti, L.H. \& Mobin, M. 2002. Myxomycetes associated with palm trees in the Sete Cidades National Park, Pauí, Brazil. Scripta Botanica Belgica 22: 19.

Cavalcanti, L.H. \& Mobin, M. 2004. Myxomycetes associated with palm trees at the Sete Cidades National Park, Piauí State, Brazil. Systematics and Geography of Plants 74: 109-127.

Cavalcanti L.H. \& Oliveira, F.C. 1985. Myxomycetes da Paraíba IV. Stemonitales. Anais VIII Reunião Nordestina de Botânica, 1984. Recife, pp. 207-214.

Cavalcanti, L.H. \& Pôrto, K.C. 1985. Trichiaceae (Myxomycetes) da floresta pluvial tropical. I. Arcyria Wiggers. Anais XXXVI Congreso Nacional de Botanica. Curitiba: 271.

Cavalcanti, L.H. \& Putzke, J. 1998. Myxomycetes da Chapada do Araripe (Crato - Ce, Brasil). Acta Botanica Brasilica 12(3): 253-261.

Cavalcanti, L.H. \& Santos, E.J. 1991. Ocorrência de Myxomycetes em folhas senescentes de cana-de-açucar (Saccharum spp.). Biologia Brasilica 3(1): 45-56.

Cavalcanti, L.H. \& Silva, J.V.B. 1985. Myxomycetes da Paraíba III. Physarales. Anais VIII Reunião Nordestina de Botânica, 1984. Recife, pp. 199-205.

Cavalcanti, L.H., Correia, A.M.S. \& Pôrto, K.C. 1982. O Herbário de Myxomycetes (Gymnomycota) da UFPE. Anais XXXIII Congresso Nacional de Botânica, Maceió, 1982, pp. 189-200.

Cavalcanti, L. H., Okada, K. \& Costa, S.P.S.E. 1993. Incidência de Myxomycetes na necromassa da mata do Curado (Recife-Pe, Brasil). Revista Nordestina Biologia 8(1): 39-44.

Cavalcanti, L.H., Ponte, M.P.M.P. \& Mobin, M. 2006. Myxomycetes, State of Piauí, Northeast Brazil. Check Lis. 2(2): 7074.

Cavalcanti, L.H., Santos, E.J. \& Gomes, N.A. 1999. Myxomycetes do estado de Roraima, com especial referência para a estação Ecológica de Maracá (Amajarí - RR, Brasil). Acta Amazônica 29: $195-200$

Cavalcanti, L.H., Santos, E.J., Silva, M.I.L. \& Pinto, I.M.A. 1985. Myxomycetes em cana-de-açúcar (Saccharum officinarum L.). Anais VIII Reunião Nordestina de Botânica, 1984. Recife, pp. 215-221.

Cavalcanti, L.H., Tavares, H.F.M., Nunes, A.T. \& Silva, C.F. 2005. Mixomicetos. In: Pôrto, K.C., Almeida Cortez, J.S. \& Tabarelli, M. (Orgs.), Diversidade biológica e conservação da Floresta Atlântica ao norte do rio São Francisco, pp. 53-74. Coll. Biodiversidade 14. Ministerio do Meio Ambiente. Brasília.

Chacón, S. \& Guzmán, G. 1984. Nuevas observaciones sobre los hongos, líquenes y mixomicetos de Chiapas. Boletín de la Sociedad Mexicana de Micología 19: 245-252.

Chardon, C.E. 1926. Scientific survey of Porto Rico and the Virgen Islands. New York Academy of Sciences, vol. Part. 3(1).

Chardon, C.E. 1928. Contribución al estudio de la Flora micológica de Colombia. Boletín de la Sociedad Española de Historia Natural 28: 111-124.

Chiappeta, A.A., Sena, K.X.R. \& Cavalcanti, L.H. 2003. Environmental Factors Affecting Sporulation of Fuligo septica (Myxomycetes) on Sugar Cane Bagasse. Brazil Archivo de Biología e Technología 46(1): 7-12.

Ciferri, R. 1929. Micoflora domingensis. Lista de hongos hasta la fecha indicados en Santo Domingo. Estación Agronómica de Moca, ser. B. Botánica 14: 1-260.

Ciferri, R. 1961. Mycoflora dominguensis integrata. Quaderno Instituto Botanico Laboratorio Crittogamico Pavia 19: 1-539.

Ciferri, R. \& González-Fragoso, R. 1926. Hongos parásitos y

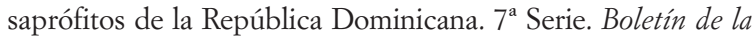
Sociedad Española de Historia Natural 26: 470-480.

Clark, J.D. \& Collins, O.R. 1973. Further studies on the genetics of plasmodial incompatibility in a Honduran isolate of Didymium iridis. Mycologia 65: 507-519.

Cooke, M.C. 1877. Cocoa-palm fungi. Grevillea 5: 101-103.

Cooke, M.C. 1889. Some exotic fungi. Grevillea 17(83): 59-60.

Courtecuise, R., Samuels, G.J., Hoff, M., Rossman, A.Y., Cremers, G., Huhndorf, S.M. \& Stephenson, S.L. 1996. Check-list of fungi from French Guiana. Mycotaxon 57: 1-85.

Crespo, E.M. \& Lugo, M. 2003. Catalogue of Myxomycetes from Argentina. Mycotaxon 87: 91-102.

Dávalos, L. \& Guzmán, G. 1981. La colección de hongos del herbario de la escuela nacional de Ciencias Biológicas del I.P.N.. Boletín de la Sociedad Mexicana de Micología 16: 105107.

Davis, E.E. \& Butterfield, W. 1967. Myxomycetes cultured from the peel of banana fruit. Mycologia 59(5): 935-937. 
Davis, S.D., Heywood, V.H., Herrera-MacBryde, O., Villa-Lobos, J. \& Hamilton, A.C. (eds.). 1997. Centres of Plant Diversity, vol. 3. Information Press, Oxford.

Dennis, R.W.G. 1960. Fungi venezuelani: III. Kew Bulletin 14(3): 418-458.

Dennis, R.W.G. 1970. Fungus flora of Venezuela and adjacent countries. Kew Bulletin Add. Ser. 3. Royal Botanic Gardens, Kew.

Deschamps, J.R. 1972. El género Stemonitis (Myxomycetidae) en Argentina. Boletín de la Sociedad Argentina de Botánica 14(3): 139-153.

Deschamps, J.R. 1974. Una nueva especie de Comatricha (Stemonitaceae-Myxomycetidae). Boletín de la Sociedad Argentina de Botánica 15(4): 340-342.

Deschamps, J.R. 1975. Los Myxomycetes de la Argentina. Catálogo crítico, distribución y clave de las especies. Physis (Buenos Aires) 34: 159-178.

Deschamps, J.R. 1976a. Los Myxomycetes de la Argentina. Catálogo crítico, distribución y clave de las especies. (Continuación). Physis (Buenos Aires) 35: 147-171.

Deschamps, J.R. 1976b. Los Myxomycetes de la Argentina. Catálogo crítico, distribución y clave de las especies. (Conclusión). Physis (Buenos Aires) 35:319-339.

Digilio, A.P.L. 1946. Contribución al catálogo de los "Myxomycetes" argentinos. I. Lilloa 12: 177-203

Digilio, A.P.L. 1950. Myxomycetes de Tucuman. Lilloa 23(4): 365-414.

Duss, A. 1903. Enumération méthodique des champignons recueillis a la Guadeloupe E a la Martinique. Impr. et lithographie L. Duclume, Lons-le-Saunier.

Duss, A. 1904. Flore cryptogamique des Antilles françaises, Champignons. Impr. et lithographie L. Duclume, Lons-leSaunier.

Edmunds, A. \& Stephenson, S.L. 1996. Myxomycetes associated with the litter microhabitat in tropical forests of Costa Rica. Proceedings of the West Virginia Academy of Sciences 68: 23-24.

Eliasson, U.H. 1971. A Collection of Myxomycetes from the Galápagos Islands. Svensk Botanisk Tidskrift 65: 105-111.

Eliasson, U.H. 2000. Myxomyceten auf lebenden Blättern im tropischen Regenwald Ecuadors; eine Untersuchung basierend auf dem Herbarmaterial höherer Pflanzen. Stapfia 73: 81-84.

Eliasson, U.H. \& Nannenga-Bremekamp, N.E. 1983. Myxomycetes of the Scalesia forest, Galápagos Islands. Proceedings of the Koninklijke Nederlandse Akademie van Wetenschappen, Series C, 86: 143-153.

Emoto, Y. 1933. Myxomyceten aus Mexiko. Botanical Magazine (Tokyo) 47: 132-135.

Estrada-Torres, A. 1996. Myxomycetes from tropical Mexico. In: Lado, C. \& Hernández, J.C. (eds.), Abstract Volume. Second International Congress on the Systematics and Ecology of Myxomycetes: 124.

Estrada-Torres, A., Gaither, T.W., Miller, D., Lado, C. \& Keller, H.W. 2005. The myxomycete genus Schenella: morphological and DNA sequence evidence for synonymy with the gasteromycete genus Pyrenogaster. Mycologia 97(1): 139-149.

Estrada-Torres, A., Hernández-Cuevas, L., Lado, C., Ramírez-Ortega, J.M. \& Díaz-Ramírez, M. 2002a. Distribución y abundancia de Myxomycetes en selvas bajas de México. In: Guzmán, G. \& Mata, G. (eds.), Estudios sobre los hongos latinoamericanos. Resumen IV Congreso Latinoamericano de Micología: 248.

Estrada-Torres, A., Lado, C. \& Flores, R. 2000. Myxomycetes de Guatemala. Stapfia 73: 159-165.
Estrada-Torres, A., Lado, C. \& Rodríguez-Palma, M.M. 2001. Two new species of Myxomycetes from a tropical deciduous forest of Mexico. Mycologia 93: 744-750.

Estrada-Torres, A., Lado, C., Stephenson, S.L., Ramírez-Ortega, M., Wrigley de Basanta, D. \& Schnittler, M. 2002b. Plant diversity in the Mexican Neotropics: opportunities for studies of ecological specificity and speciation in myxomycetes. Scripta Botanica Belgica 22: 27-28.

Estrada-Torres, A., Lado, C., Stephenson, S.L., Wrigley de Basanta, D. \& Schnittler, M. 2002c. Myxomycetes de tres reservas biológicas neotropicales. Análisis de resultados. In: Guzmán, G. \& Mata, G. (eds.), Estudios sobre los hongos latinoamericanos. Resumen IV Congreso Latinoamericano de Micología: 249.

Estrada-Torres, A., Márquez, J., Esquivel, C., Ramírez, M. \& Lado, C. 2002d. Morphogenesis of the sporocarps of Cribraria fragilis. Scripta Botanica Belgica 22: 29.

Estrada-Torres, A., Ramírez-Ortega, J.M. \& Lado, C. 2003. Calonema foliicola a new myxomycete from Mexico. Mycologia 95: 354-359.

Estrada-Torres, A. Wrigley de Basanta, D., Conde, E. \& Lado, C. (in press). Myxomycetes associated with dryland ecosystems of the Tehuacán-Cuicatlán Valley Biosphere Reserve, Mexico. Fungal Diversity.

Farr, M.L. 1957. A Checklist of Jamaican Slime-Moulds (Myxomycetes). Bulletin Institute Jamaica, Sciences Serie 7: 1-67.

Farr, M.L. 1958. Taxonomic studies in the Myxomycetes I. The Trichia favoginea complex. Mycologia 50(3): 357-369.

Farr, M.L. 1959. Stemonitis brasiliensis and Badhamia Iowensis-A correction. Mycologia 51(4): 598.

Farr, M.L. 1960. The Myxomycetes of the IMUR herbarium, with special reference to Brazilian species. Instituto Micologia Universidade de Recife 184: 1-54.

Farr, M.L. 1967. Notes on Myxomycetes. Mycopathologia et Mycologia Applicata 31(3/4): 305-313.

Farr, M.L. 1968. An illustrated key to the Myxomycetes of South America, with special reference to Brasil. Rickia 3: 45-88.

Farr, M.L. 1969. Myxomycetes from Dominica. Contributions U. S. National Herbarium 37(6): 397-440.

Farr, M.L. 1971. Two undescribed Myxomycetes from Argentina. Mycologia 63(3): 634-639.

Farr, M.L. 1973. An annotated list of Spegazzini's fungus taxa. Bibliotheca Mycologica 35, 2 vols.

Farr, M.L. 1974. Some new Myxomycete records for the Neotropics and some taxonomic problems in the Myxomycetes. Proceedings of the Iowa Academy of Sciences 81: 37-40.

Farr, M.L. 1976. Flora Neotropica Monograph No. 16 (Myxomycetes). New York Botanical Garden, New York.

Farr, M.L. 1985. Notes on Myxomycetes. IV. Species collected in Brazil and Japan. Nova Hedwigia 41: 167-176.

Farr, M.L. \& Kowalski, D.T. 1974. A new species of Calomyxa from the Andes. Mycologia 66: 884-886.

Farr, M.L. \& Martin, G.W. 1958. Two new Myxomycetes from Brazil. Brotéria 27(4): 153-158.

Farr, M.L., Eliasson, U. \& Dumont, K.P. 1979. Myxomycetes from Ecuador. Mycotaxon 8: 127-134.

Fidalgo, O., Fidalgo, M.E.K. \& Furtado, J.S. 1965. Fungi of the "Cerrado" region of São Paulo. Rickia 2: 55-71.

Fries, R.E. 1903. Myxomyceten von Argentinien und Bolivia. Arkiv für Botanik 1: 57-70

Fries, R.E. 1920. Die Myxomyceten der Juan Fernandez Inseln. In: Skottsberg, C. (ed.), The natural history of Juan Fernandez and Easter Islands. Upsala 2(1): 55-58. 
Galindo-Flores, G.L. 1992. Algunos hongos del Jardín Botánico Tizatlán, Tlaxcala. Talleres gráficos del Estado de Tlaxcala. Tlaxcala.

Galindo-Flores, G.L. \& Estrada-Torres, A. 1993. Algunas consideraciones taxonómicas y biogegráficas del género Arcyria del Volcán La Malintzi, Tlaxcala. In: XII Congreso de Botánica. Libro de resúmenes: 100.

Galindo-Flores, G.L., Hernández-Cuevas, L., Rodríguez-Palma, M. \& Estrada-Torres, A. 1993. Contribución al conocimiento de los mixomicetos del parque nacional Lagunas de Zempoala. Acta Botánica Mexicana 21: 27-42.

Gams, W. 2005. Report of the Committee for Fungi: 13. Taxon 54(3): 828-830

García-Zorrón, N. 1967. Mixomicetos del Uruguay. Fac. Humanidades y Ciencias Dep. Botanica Univ. República Montevideo.

García-Zorrón, N. 1977. Mixomicetos coprófilos del Uruguay. Revista de Biología Uruguaya 5: 47-50.

Gentry, A.H. 1982. Neotropical floristic diversity. Annales Missouri Botanical Garden 69: 557-593.

Gentry, A.H. 1992. Tropical Forest Biodiversity. Oikos 63: 19-28.

GBIF-Sweden, Gothenburg Herbarium - General (GBIF:IH:GB: Herbarium) (accessed through GBIF data portal, http: //data.gbif.org/datasets/resource/1765, 2008-06-24).

Gilbert, F.A. 1928. Myxomycetes from British Guiana and Surinam. Mycologia 20(1): 27-28.

Góes Neto, A. 1996. Biodiversidade de mixomicetos e fungos macroscópicos da Reserva Biológica de Una e áreas adjacentes (Bahia, Brasil). Sitientibus 15: 91-107.

Góes Neto, A. \& Cavalcanti, L.H. 2002. Myxomycetes of the State of Bahia, Brazil: historical rewieve and current situation. Mycotaxon 82: 335-342.

Gómez-Pompa, A., Moreno, N.P., Gama, L. \& Sosa, V. 1984. Flora of Veracruz: Progress and Prospects. In: Allkin, R. \& Bisby, F.A. (eds.), Databases in Systematics. The Systematics Association Special Volume 26: 165-174.

Gómez-Sánchez, A. \& Castillo, J. 1981. Estudio sobre los myxomycetes del Estado de Nuevo León. Boletín de la Sociedad Mexicana de Micología 15: 193-223.

González-Fragoso, R. \& Ciferri, R. 1927. Hongos parásitos y saprofitos de la República Dominicana. Ser. 6-10. Estación Agronómica de Moca, ser. B. Botánica 8: 1-99.

González-Fragoso, R. \& Ciferri, R. 1928. Hongos parásitos y saprofitos de la República Dominicana. Ser. 16. Boletín de la Sociedad Española de Historia Natural 28: 377-388.

Gottsberger, G. 1968. Myxomycetes aus Bahia und Goiás. Nova Hedwigia 15:361-368.

Gottsberger, G. 1971. Myxomyceten auf Bromeliaceen. Nova Hedwigia 22 (1,2): 489-501.

Gottsberger, G. \& Nannenga-Bremekamp, N.E. 1971. A new species of Didymium from Brazil. Proceedings of the Koninklijke Nederlandse Akademie van Wetenschappen, Series $C$ 74(3): 264-268

Gottsberger, G., Schmidt, I. \& Meijer, A.R. 1992. Myxomycetes from the state of Paraná-Brasil 2. Arquivos de Biologia e Tecnologia 33: 631-633.

Guzmán, G. 1972. Algunos macromicetos, líquenes y mixomicetos importantes de la zona del Volcán Popocatépetl. Guías Botánicas de excursiones en México. Sociedad Botánica de México pp. 17-44.

Guzmán, G. 1983. Los hongos de la Península de Yucatán. II. Nuevas exploraciones y adiciones micológicas. Biótica 8: 71100 .
Guzmán, G. \& Guzmán Dávalos, L. 1981. Nuevos datos sobre el myxomycete Physarum pusillum en México. Boletín de la Sociedad Mexicana de Micología 16: 105-107.

Guzmán, G. \& Varela, L. 1978. Los hongos de Colombia. III. Observaciones sobre los hongos, líquenes y Mixomicetos de Colombia. Caldasia 12(58): 309-338.

Guzmán, G. \& Varela, A. 1979. Hongos del herbario de W.B. Cooke (E.U.A.) depositados en ENCB y discusiones sobre su distribución en México. Boletín de la Sociedad Mexicana de Micología 13: 63-73.

Guzmán, G. \& Villarreal, L. 1984. Estudio sobre los hongos, líquenes y mixomicetos del Cofre de Perote, Veracruz I. Introducción a la Micoflora de la región. Boletín de la Sociedad Mexicana de Micología 19: 107-124.

Hagelstein, R. 1927. Mycetozoa from Porto Rico. Mycologia 19(1): 35-37.

Hagelstein, R. 1932. Revision of the Myxomycetes. In Seaver \& Chardon, Scientific survey of Porto Rico and the Virgin Islands. New York Academy of Sciences Annual 8(2): 241-248.

Hagelstein, R. 1944. The Mycetozoa of North America. Hagelstein. Mineola, New York.

Harling, G. 1967. Notes on Myxomycetes.II. Species collected in Ecuador 1958-59. Svensk Botanisk Tidskrift 61(1): 139-144.

Hashimoto, G. 1953. Myxomycetes da Serra do Diabo. Revista Ceres 9(51): 194-201.

Heim, R. 1928. Champignons recueillis par M Mayeul Grisol dans le haut Orénique. Annales de Cryptogamie Exotique 1(3): 266278.

Hennings, P. 1896. Beiträge zur Pilzflora Südamerikas I. Myxomycetes, Phycomycetes, Ustilagineae und Uredinae. Hedwigia 35: 202-262.

Hennings, P. 1902a. Fungi paraenses. (II). Beibl. Hedwigia 41(1): 15-18.

Hennings, P. 1902b. Fungi costaricenses I. Beibl. Hedwigia 41(3): 101-105.

Heredia, G. 1989. Estudio de los hongos de la reserva de la Biosfera El Cielo, Tamaulipas. Consideraciones sobre la distribución y ecología de algunas especies. Acta Botánica Mexicana 7: 1-18.

Hernández-Crespo, J.C. \& Lado, C. 2005. An on-line nomenclatural information system of Eumycetozoa. http: //www.nomen. eumycetozoa.com (10-XI-2007).

Hernández-Cuevas, L. \& Estrada-Torres, A. 1993a. Distribución espacio-temporal de las especies del género Physarum en el Volcán La Malintzin, Tlaxcala. In: XII Congreso Mexicano de Botánica. Libro de resúmenes: 124.

Hernández-Cuevas, L. \& Estrada-Torres, A. 1993b. El género Badhamia en el estado de Tlaxcala. In: XII Congreso Mexicano de Botánica. Libro de resúmenes: 125.

Hernández-Cuevas, L. \& A. Estrada-Torres 1997. Mexican Didymiaceae (Myxomycetes, order Physarales): Three new records and comments on Mucilago crustacea. Mycotaxon 62: 319-335.

Hernández-Cuevas, L., Rodríguez-Palma, M., Galindo-Flores, G. \& Estrada-Torres, A. 1991. New records of myxomycetes from Mexico. Mycotaxon 62: 17-27.

Hertel, R.J.G. 1954a. Myxomycetes do Brasil I. Dusenia 5(2): 117 124.

Hertel, R.J.G. 1954b. Myxomycetes do Brasil II. Paradiacheopsis curitibana Hertel, n. gen. e n. sp. de Lamprodermaceae. Dusenia 5(3-4): 191-192.

Hertel, R.J.G. 1955. Myxomycetes do Brasil III. Dois novos elementos de Stemonitaceae. Dusenia 6(1-2): 47-48. 
Herter, W.G. 1907. Hongos coleccionados en la República Oriental del Uruguay. Revista Agronómica Universidad de Montevideo 2: 144-152.

Herter, W.G. 1933. Florula Uruguayensis. Plantae avasculares. Ostenia: 7-84.

Herter, W.G. 1939. Plantae Uruguayensis novae vel criticae. Pars II. Revista Sudamericana de Botánica 6(3-4): 69-107.

Hochgesand, E. \& Gottsberger, G. 1989. Arcyriatella congregata, a new genus and species of the Trichiaceae (Myxomycetes). Nova Hedwigia 48: 485-489.

Hochgesand, E. \& Gottsberger, G. 1996. Myxomycetes from the State of São Paulo, Brazil. Boletim Instituto de Botanica 10: 1-46.

Hochgesand, E., Gottsberger, G. \& Nannenga-Bremekamp, N.E. 1989. A new species and a new variety of Didymium from São Paulo State, Brazil. Proceedings of the Koninklijke Nederlandse Akademie van Wetenschappen, Series C 92: 73-79.

Höhnel, F. von. 1907. Ergebnisse der botanischen Expedition der Kaiserlichen Akademie der Wissenschaften nach Südbrasilien 1901. Eumycetes et Myxomycetes. Denkschriften der Kaiserlichen Akademie der Wissenschaften Mathematisch-naturwissenschaftliche Klasse 83: 1-45

Illana, C. 1996. Myxomycetes de Baja California. In: Lado, C. \& Hernández, J.C. (eds.), Abstract Volume. Second International Congress on the Systematics and Ecology of Myxomycetes: 125.

Illana, C., Moreno, G. \& Lizárraga, M. 2000. Catálogo de Myxomycetes de México. Stapfia 73: 167-186.

Illana, C., Moreno, G., Lizarraga, M. \& Castillo, A. 1999. Hemitrichia pseudoleiocarpa, spec. nova, a species confused with Arcyria leiocarpa (Myxomycetes). Österreichische Zeitschrift für Pilzkunde 8: 63-70.

Ing, B. 1967. Notes on Myxomycetes.II. Transactions British Mycological Society 50(4): 555-562.

Ing, B. 1994. The phytosociology of Myxomycetes. New Phytologist 126: 175-201.

Ing, B. 1999. The Myxomycetes of Britain and Ireland. An identification handbook. The Richmond Publishing Co. Ltd. Slough, England.

Ing, B. \& Haynes, C. 1999. Corticolous myxomycetes from Belize. Kew Bulletin 54: 723-730.

Jahn, E. 1902. Myxomycetenstudien.2. Arten aus Blumenau (Brasilien). Berichte der Deutschen Botanischen Gesellschaft 20: 268-280.

Jahn, E. 1904. Myxomyceten aus Amazonas. Gesammelt von E.Ule. Hedwigia 43: 300-305.

Johow, F.R.A. 1896. Estudios sobre la flora de las Islas de Juan Fernández. Imprenta Cervantes. Santiago de Chile.

Keller, H.W. \& Braun, K.L. 1977. Myxomycetes of Mexico II. Boletín de la Sociedad Mexicana de Micológica 11: 167-180.

Keller, H.W. \& Brooks, T.E. 1976. Corticolous Myxomycetes V: Observations on the genus Echinostelium. Mycologia 68: 12041220.

Klotzsch, J.F. 1852. Fungi Portoricenses quos Schwanecke, hortulanus, collegit et cl. Dr. Klotzsch examinavit. In: Schlechtendal, D.F.L. Beitrag zur Flora der Insel Portorico. Anlage No. 3. Linnaea 25(3): 364-366.

Krivomaz, T.I. 2003. Addition to Cuban Myxomycetes from international collaboration. XIV Congress of European Mycologist, abstracts.

Lado, C. 1994. A checklist of Myxomycetes of the Mediterranean countries. Mycotaxon 52: 117-185.

Lado, C. 2001. NOMENMYX. A nomenclatural taxabase of Myxomycetes. Cuadernos de Trabajo de Flora Micológica Ibérica 16: $1-221$.
Lado, C. 2008. Eumycetozoa.com: nomenclatural Database of Eumycetozoa (Myxomycota) (Oct 2007 version). In Species 2000 \& ITIS Catalogue of Life: 2008 Annual Checklist (Bisby FA, Roskov YR, Orrell TM, Nicolson D, Paglinawan LE, Bailly N, Kirk, PM, Bourgoin T van Hertum J, eds). CD-ROM; Species 2000: Reading, U.K.

Lado, C., Eliasson, U., Stephenson, S.L., Estrada-Torres, A. \& Schnittler, M. 2005. (1688-1691) Proposals to conserve the names Amaurochaete against Lachnobolus, Ceratiomyxa against Famintzinia, Cribraria Pers. against Cribraria Schrad. ex J.F. Gmel. and Hemitrichia against Hyporhamma (Myxomycetes). Taxon 54(2): 543-545.

Lado, C., Estrada-Torres, A., Ramírez, M. \& Conde, E. 2002a. A study of the succulenticolous Myxomycetes from arid zones of Mexico. Scripta Botanica Belgica 22: 58.

Lado, C., Estrada-Torres, A., Schnittler, M., Wrigley, D. \& Stephenson, S.L. 2002b. Rapid biodiversity assessment of the Myxomycetes from the Yasuni Biosphere Reserve (Ecuador). Scripta Botanica Belgica. 22: 59-60.

Lado, C., Estrada-Torres, A. \& Stephenson, S.L. 2007a. Myxomycetes collected in the first phase of a north-south transect of Chile. Fungal Diversity 25: 81-101.

Lado, C., Estrada-Torres, A., Stephenson, S.L., Wrigley de Basanta, D. \& Schnittler, M. 2003. Biodiversity assessment of myxomycetes from two tropical forest reserves in Mexico. Fungal Diversity 12: 67-110.

Lado, C., Mosquera, J. \& Beltrán-Tejera, E. 1999a. Cribraria zonatispora, development of a new myxomycete with unique spores. Mycologia 91: 157-165.

Lado, C., Mosquera, J., Estrada-Torres, A., Beltrán-Tejera, E. \& Wrigley de Basanta, D. 2007b. Description and culture of a new succulenticolous Didymium (Myxomycetes). Mycologia 99(4): 602-611.

Lado, C., Rodríguez-Palma, M. \& Estrada-Torres, A. 1999b. Myxomycetes from a seasonal tropical forest on the Pacific coast of Mexico. Mycotaxon 71: 307-321.

Lazo, W.R. 1966. Notes and illustrations of Myxomycetes from Chile and other countries. Mycologia 58(1): 67-79.

Léveillé, J.H. 1863. Fungi. In: Triana, J. \& Planchon, J.E. Prodromus Florae Novo-Granatensis. Annales des Sciences Naturalles IV Botanique 20(5): 282-300.

Ling, H. \& Collins, O.R. 1970. Control of plasmodial fusion in a Panamanian isolate of Didymium iridis. American Journal of Botany 57: 292-298.

Lister, A. 1894. A Monograph of the mycetozoa. Printed by order of the Trustees. London.

Lister, A. 1898a. Mycetozoa of Antigua and Dominica. Journal of Botany 36: 113-122.

Lister, A. 1898b. Mycetozoa of Antigua. Journal of Botany 36: 378 379.

Lister, A. 1911. A monograph of the Mycetozoa. $2^{\mathrm{a}}$ ed. revisada por G. Lister. Printed by order of the Trustees. London.

Lister, A. 1925. A monograph of the Mycetozoa, $3^{\text {a }}$ ed., revisada por G. Lister. Printed by order of the Trustees. London.

Lizárraga, M. 2002. Myxomycetes de Chihuahua I. In: Guzmán, G. \& Mata, G. (eds.), Estudios sobre los hongos latinoamericanos. Resumen IV Congreso Latinoamericano de Micología: 250.

Lizárraga, M., Illana, C. \& Moreno, G. 1998. Didymium subreticulosporum (myxomycetes), a new species for America. Mycotaxon 67:313-316.

Lizárraga, M., Illana, C. \& Moreno, G. 1999a. First records of myxomycete in the state of Sinaloa, Mexico. Micologia Vegetacione Mediterranea 13: 167-176. 
Lizárraga, M., Illana, C. \& Moreno, G. 1999b. SEM studies of the myxomycete from the Peninsula of Baja California (Mexico), I. Arcyria to Fuligo. Annales Botanici Fennici 35: 287-306.

Lizárraga, M., Illana, C. \& Moreno, G. 1999c. SEM studies of the myxomycete from the Peninsula of Baja California (Mexico), II. Hemitrichia to Trichia. Annales Botanici Fennici 36: 187-210.

Lizárraga, M., Illana, C. \& Moreno, G. 2004a. Contribución al estudio de los Myxomycetes de la península de Baja California, México. Boletín de la Sociedad Micológica de Madrid 28: 45-53.

Lizarraga, M., Moreno, G. \& Illana, C. 1997. The myxomycetes from Baja California (Mexico). I. Mycotaxon. 63: 287-300.

Lizárraga, M., Moreno, G. \& Illana, C. 2005a. Myxomycetes from Chihuahua, Mexico. 2. Österreichische Zeitschrift für Pilzkunde 14: 105-121.

Lizárraga, M., Moreno, G. \& Illana, C. 2006. Macbrideola herrerae sp. nov., a new myxomycete from Mexico. Boletín de la Sociedad Micológica de Madrid 30: 265-269.

Lizárraga, M., Moreno, G., Esqueda, M. \& Coronado, M.L. 2008. Myxomycetes of Sonora, Mexico. 4: Sierra de Alamos-Rio Cuchujaqui Biosphere Reserve. Mycotaxon 103: 153-170.

Lizárraga, M., Moreno, G., Esqueda, M., Sánchez, A. \& Coronado, M. 2007. Myxomycetes from Sonora, Mexico. 3: National Forest Reserve and Wildlife Refuge, Ajos-Bavispe. Mycotaxon 99: 291-301.

Lizárraga, M., Moreno, G., Illana, C. \& Castillo, A. 1996. Two new species of Myxomycetes from Mexico. In: Lado, C. \& Hernández, J.C. (eds.), Abstract Volume. Second International Congress on the Systematics and Ecology of Myxomycetes: 56.

Lizárraga, M., Moreno, G., Illana, C. \& Singer, H. 2004b. Calonema foliicola, a myxomycete with a difficult taxonomic position. Micologia Vegetacione Mediterrane 19(1): 38-42.

Lizárraga, M., Moreno, G., Illana, C. \& Singer, H. 2005b. Myxomycetes from Chihuahua, Mexico III. Mycotaxon 93: 75-88.

Lizarraga, M., Moreno, G., Illana, C. \& Solis, F. 2003a. Myxomycetes in the State of Sinaloa (Mexico) II. Mycotaxon 88: 425-432.

Lizarraga, M., Moreno, G., Singer, H. \& Illiana, C. 2003b. Myxomycetes from Chihuahua, Mexico. Mycotaxon 88: 409-424.

López, A. \& García, J. 1996a. Didymium squamulosum (Alb. \& Schw.) Fries. Funga Veracruzana 10.

López, A. \& García, J. 1996b. Physarella oblonga (Berk. \& Curt.) Morgan. Funga Veracruzana 11.

López, A. \& García, J. 1996c. Didymium verrucosporum Welden. Funga Veracruzana 12.

López, A. \& García, J. 1996d. Didymium ovoideum Nann.-Brem. Funga Veracruzana 13.

López, A. \& García, J. 1996e. Didymium nigripes (Link) Fr. Funga Veracruzana 14.

López, A. \& García, J. 1996f. Didymium minus (A. Lister) Morgan. Funga Veracruzana 15.

López, A. \& García, J. 1996g. Didymium clavus (Alb. \& Schw.) Rab. Funga Veracruzana 16.

López, A. \& García, J. 1996h. Didymium megalosporum Berk. \& Curt. Funga Veracruzana 17.

López, A. \& García, J. 1996i. Didymium leoninum Berk. \& Br. Funga Veracruzana 18.

López, A. \& García, J. 1996j. Didymium iridis (Ditmar) Fries. Funga Veracruzana 20.

López, A. \& García, J. 2001a. Dictydium cancellatum. Funga Veracruzana 30.

López, A. \& García, J. 2001b. Physarum pezizoideum. Funga Veracruzana 32.

López, A. \& García, J. 2001c. Hemitrichia serpula. Funga Veracruzana 48.
López, A. \& García, J. 2001d. Trichia scabra. Funga Veracruzana 49.

López, A. \& García, J. 2002a. Physarum compressum. Funga Veracruzana 51

López, A. \& García, J. 2002b. Hemitrichia calyculata. Funga Veracruzana 53

López, A. \& García, J. 2002c. Lycogala epidendrum. Funga Veracruzana 55 .

López, A. \& García, J. 2002d. Diderma hemisphaericum. Funga Veracruzana 60.

López, A. \& García, J. 2002e. Arcyria incarnata. Funga Veracruzana 65.

López, A. \& García, J. 2002f. Arcyria nigella. Funga Veracruzana 66.

López, A. \& García, J. 2002g. Arcyria cinerea. Funga Veracruzana 73.

López, A. \& García, J. 2005a. Physarum virescens. Funga Veracruzana 85.

López, A. \& García, J. 2005b. Didymium anellus. Funga Veracruzana 90.

López, A. \& Sosa, A. 1982. Myxomycetes del estado de Quintana Roo. 1er Congreso Nacional de Micología, Xalapa, Veracruz. Libro de Resúmenes: 7.

López, A., Sosa, A. \& Villarreal, L. 1979. Estudio sobre los hongos myxomycetes del Estado de Veracruz. I. Boletín de la Sociedad Mexicana de Micología 13: 127-144.

López, A., Sosa, A. \& Villarreal, L. 1981a. Estudio sobre los hongos myxomycetes del Estado de Veracruz. II. Biótica 6: 43-56.

López, A., Sosa, A. \& Villarreal, L. 1982. Estudios sobre los Myxomycetes de Veracruz. IV. 1er Congreso Nacional de Micología, Xalapa, Veracruz. Libro de Resúmenes: C-95.

López, A., Villarreal, L. \& Sosa, A. 1981b. Estudio sobre los hongos myxomycetes del Estado de Veracruz. III. Boletín de la Sociedad Mexicana de Micología 16: 77-94.

López, A., Villarreal, L. \& Sosa, A. 1981c. Estudio sobre los hongos myxomycetes del Estado de Veracruz. V. Los myxomycetes mexicanos registrados en la literatura. Boletín de la Sociedad Mexicana de Micología 16: 95-104.

Macbride, T.H. 1893. Nicaraguan myxomycetes. Bulletin of the Laboratories of Natural History, Iowa State University 2(2): 277 283.

Macbride, T.H. 1899. The North American slime-moulds. Macmillan and Co., Ltd. New York.

Macbride, T.H. 1922. The North American slime-moulds (ed. 2). Macmillan and Co., Ltd. New York.

Macbride, T.H. \& Smith, C.L. 1896. The Nicaraguan Myxomycetes. With notes on certain Mexican species (continued). Bulletin of the Laboratories of Natural History, Iowa State University 4(1): 73-75.

Maimoni-Rodella, R.C. 2002. Biodiversidade e distribuição de mixomicetos em ambientes naturais e antropogênicos no Brasil: espécies ocorrentes nas Regiões Sudeste e Centro-Oeste. In: Araújo, E.L., Moura, A.N., Sampaio, E.V.S.B., Gestinari, L.M.S. \& Carneiro, J.M.T. (eds.), Biodiversidade, conservação e uso sustentável da flora do Brasil, pp. 217-220. Universidade Federal Rural de Pernambuco, Sociedade Botânica do Brasil, Recife.

Maimoni-Rodella, R. \& Cavalcanti, L.H. 2006. Myxomycetes sobre inflorescências e folhas vivas de lírio-do-brejo (Hedychium coronarium Koenig, Zingiberaceae): registro de um novo substrato. Revista Brasileira de Botanica 29(2):331-333.

Maimoni-Rodella, R.C. \& Gottsberger, G. 1980. Myxomycetes from the forest and the cerrado vegetation in Botucatu, Brazil: A comparative ecological study. Nova Hedwigia 34: 207-246. 
Mapes, C., Guzmán, G. \& Caballero, J. 1981. Etnomicología purépecha. El conocimiento y uso de los hongos de la cuenca del lago Pátzcuaro, Michoacán. Cuadernos de Etnobiología 2.

Mariz, G. 1968. Gêneros de Mixomicetes de ocorrência em Pernambuco. Universidade Federal de Pernambuco, Recife.

Mariz, G. \& Cavalcanti, L.H. 1970. Alguns Mixomicetos do Pernambuco. Instituto de Biociências, Botanica ser. B 1(5): 1-9.

Martin, G.W. 1932. New species of slime molds. Journal of the Washington Academy of the Sciences 22(4): 88-92.

Martin, G.W. 1936. Myxomycetes from Panama. Transactions of the American Microscopical Society 55: 277-280.

Martin, G.W. 1938a. Myxomycetes from Colombia. Transactions of the American Microscopical Society 57: 123-126.

Martin, G.W. 1938b. Additional Myxomycetes from Panamá. Studies in Natural History, Iowa University 17(8): 347-350.

Martin, G.W. 1948. Additions to Galapagos fungi. Pacific Sciences 2: 71-77.

Martin, G.W. 1957. A new species of Licea from Panama. Mycologia 49: 439-440.

Martin, G.W. \& Alexopoulos, C.J. 1969. The Myxomycetes. Univ. Iowa Press. Iowa

Martínez-Alfaro, M.A., Pérez-Silva, E. \& Aguirre-Acosta, E. 1983. Etnomicología y exploraciones micológicas en la Sierra Norte de Puebla. Boletín de la Sociedad Mexicana de Micología 18: 51-63.

Martínez-Murillo, M.A. \& López-Ochoterena, E. 1970. Contribución al conocimiento de los micetozoarios (Protozoo, Sarcodina) de Mexico. Revista de la Sociedad Mexicana de Historia Natural 31: 95-106.

Massee, G. 1889. A revision of the Trichiaceae. Journal of the Royal Microscopical Society of London 1889(1): 325-359.

Matsumoto, J. 2002. Taxonomic study on Didymium floccoides. Scripta Botanica Belgica 22: 63.

McHugh, R. 2005. Moist chamber cultura and field collections of Myxomycetes from Ecuador. Mycotaxon 91: 107-118.

Mendes, C.L. \& Guerrero, R.T. 1990. Myxomycetes do Morro Santana, Porto Alegre, Rio Grande do Sul. Boletim Instituto de Biociencias de la Universidade Federal de Río Grande do Sul 46: 1- 95.

Minter, D.W., Rodríguez Hernández, M. \& Mena Portales, J. 2001. Fungi of the Caribbean. An annotated checklist. PDMS Publishing, Middlesex.

Mittermeier, R.A., Robles Gil, P., Hoffmann, M., Pilgrim, J., Brooks, T., Mittermeier, C.G., Lamoreux, J. \& Fonseca, G.A.B. da. 2004. Hotspots Revisited. Cemex. México.

Mobin, M. \& Cavalcanti, L.H. 1998. Myxomycetes ocorrentes sobre buriti (Mauritia venifera L. f; Arecaceae). Revista da Universidade do Amazonas, Sér. Ciencias Biologicas 2/3: 43-51.

Mobin, M. \& Cavalcanti, L.H. 1999a. Physarales (Myxomycetes) do Parque Nacional de Sete Cidades (Piauí, Brasil). Hoebnea 26: 1-14.

Mobin, M. \& Cavalcanti, L.H. 1999b. Stemonitales (Myxomycetes) do Parque Nacional de Sete Cidades (Piauí, Brasil). Acta Botanica Brasílica 13: 139-148.

Mobin, M. \& Cavalcanti, L.H. 2000. Myxomycetes em Carnaúbeira (Copernicia prunifera (Miller) T.E. Moore, Arecaceae). Acta Botanica Brasílica 14: 71-75.

Mobin, M. \& Cavalcanti, L.H. 2001. Trichiaceae (Myxomycetes) do Parque Nacional de Sete Cidades (Piripiri - Piauí - Brasil). Hoebnea 28(1): 39-51.

Montagne, C. 1837. Centurie de plantes cellulaires exotiques nouvelles. Ser. II. Annales des Sciences Naturelles. Paris 8 :345-370.

Montagne, C. 1838. Botanique. Plantes cellulaires. In: Ramón de la Sagra, M. Histoire Physique, Politique et Naturelle de l'ille de Cuba. Paris.
Montagne, J.P.F.C. 1852a. Botanica. In C. Gay, Historia fisica y politica de Chile. Paris

Montagne, J.P.F.C. 1852b. Hongos. In: Gay, C., Flora Chilensis 8: $1-53$.

Montagne, J.P.F.C. 1855. Cryptogamie Guyanensis. Annales des Sciences Naturelles. IV 3(2): 91-145.

Morawetz, W. \& Readig, C. 2007. Angiosperms biodiversity. Endemism and conservation in the Neotropics. Taxon 56(4): 1245-1254.

Moore, D.L. \& Stephenson, S.L. 2003. Microhabitat distribution of protostelids in a Tropical Wet Forest in Costa Rica. Mycologia 95(1): 11-18.

Moreno, G., Castillo, A., Illana, C. \& Lizárraga, M. 1997a. Taxonomic status of Didymium laxifilum and D. rubeopus, incl. a new variety of D. rubeopus (myxomycetes). Cryptogamie $M y$ cologie 18: 315-325.

Moreno, G., Illana, C. \& Lizárraga, M. 2001. SEM studies of the Myxomycetes from the Peninsula of Baja California (Mexico), III. Additions. Annales Botanici Fennici 38: 225-247.

Moreno, G., Illana, C., Esqueda, M., Castillo, A. \& Pérez-Silva, E. 2004. Notes on Myxomycetes from Mexico. II. Boletín de la Sociedad Micológica de Madrid 28: 55-63.

Moreno, G., Lizárraga, M. \& Illana, C. 1997b. Metatrichia horrida (myxomycetes), an African species in the Baja California Peninsula (Mexico). Mycotaxon 64: 385-392.

Moreno, G., Lizárraga, M. \& Illana, C. 1997c. A rare Didymium from Mexico (Myxomycetes). Cryptogamie Mycologie 18: 327-331.

Moreno, G., Lizárraga, M. \& Illana, C. 2006a. Macbrideola lamprodermoides sp. nov., a new myxomycete from Mexico. Boletín de la Sociedad Micológica de Madrid 30: 255-263.

Moreno, G., Lizárraga, M. \& Illana, C. 2007. Catálogo de los Myxomycetes de México. Boletín de la Sociedad Micológica de Madrid 31: 187-229.

Moreno, G., Lizárraga, M., Esqueda, M., Pérez-Silva, E. \& Herrera, T. 2006b. Myxomycetes de Sonora, México. II: Reserva Forestal Nacional y Refugio de Fauna Silvestre Ajos-Bavispe. Revista Mexicana de Micología 22: 13-23.

Moreno, G., Lizárraga, M., Illana, C., Castillo, A. \& Oltra, M. 2000. Hemitrichia agaves sp. nov. un nuovo Myxomycetes delle piante grasse dal Messico e dalla Spagna. Rivista Micologica Boletín della Associaccione Micologica Bresadola 21: 5-16.

Mosquera, J., Lado, C. \& Beltrán-Tejera, E. 2000a. Morphology and ecology of Didymium subreticulosporum. Mycologia 92: 978-983.

Mosquera, J., Lado, C., Estrada-Torres, A. \& Beltrán-Tejera, E. 2000b. Trichia perichaenoides, a new myxomycete associated with decaying succulent plants. Mycotaxon 75:319-328.

Mosquera, J., Lado, C., Estrada-Torres, A., Beltrán-Tejera, E. \& Wrigley de Basanta, D. 2003. Description and cultura of a new myxomycete Licea succulenticola. Anales del Jardín Botánico de Madrid 60: 3-10.

Muchovej, J.J. \& Muchovej, J.R.M.C. 1987. Physarum cinereum on turfgrass in Brazil. Fitopatologia Brásiliensis 12: 402-403.

Muenscher, W.C. 1930. Myxomycètes. In: Chardon and Toro. Mycological explorations of Colombia. Journal of the Department of Agriculture of Porto Rico 14(4): 214-215.

Muenscher, W.C. 1934. Myxomycetes. In Chardon \& Toro. Mycological explorations of Venezuela. Monografías Universidad de Puerto Rico, B 2: 71-75.

Mújica, F. \& Vergara, C. 1945. Flora Fungosa Chilena. Ministerio de Agricultura. Imprenta Stanley. Santiago.

Myers, N. Mittermeier, R.A. Mittermeier, C.G., Fonseca, G.A. \& Kent, J. 2000. Biodiversity hotspots for conservation priorities. Nature 403: 853-858. 
Nannenga-Bremekamp, N.E. 1961. Notes on Myxomycetes III. A new species from Suriname. Acta Botanica Neerlandica 10:54-55.

Nannenga-Bremekamp, N.E. 1989. Notes on Myxomycetes XXIII. Seven new species of Myxomycetes. Proceedings of the Koninklijke Nederlandse Akademie van Wetenschappen, series C, 92: 505-515.

Nannenga-Bremekamp, N.E. 1991. A guide to temperate Myxomycetes. Biopress Limited. Bristol.

Nieves-Rivera, A.M. 2003. Mycological survey of Río Camuy Caves Park, Puerto Rico. Journal Cave Karst Studies 65(1): 23-28.

Nieves-Rivera, A.M. \& Darrah, R.G. 2002a. The search for Myxomycetes and Protostelids in Puerto Rico. Inoculum 53(2): 8-10.

Nieves-Rivera, A.M. \& Darrah, R.G. 2002b. Further studies of slime molds in Puerto Rico. Inoculum 53(5): 2-5.

Novozhilov, Y.K., Schnittler, M., Rollins, A.W. \& Stephenson, S.L. 2000. Myxomycetes from different forest types in Puerto Rico. Mycotaxon 77: 285-299.

Ogata, N. \& Andrade-Torres, A. 1996. Los Myxomycetes de la Reserva Ecológica "ElEdén" Quintana Roo, Mexico. web www.ucr.edu

Ogata, N., Nestel, D., Rico-Gray, V. \& Guzmán, G. 1994. Los Myxomycetes citados de México. Acta Botánica Mexicana 27: $39-52$

Ogata, N., Rico-Gray, V. \& Nestel, D. 1996. Abundance, richness, and diversity of Myxomycetes in a Neotropical Forest Ravine. Biotropica 28: 627-635.

Pando, F. 1997. Catálogo preliminar de los Mixomicetes del Parque Nacional de Coiba (Panamá). In: Castroviejo, S. (ed.), Flora y fauna del Parque Nacional de Coiba (Panamá), pp. 191-204. Agencia Española de Cooperación Internacional. Madrid.

Patouillard, N. \& Gaillard, A. 1888. Champignons du Venezuela et du Haut-Orenoque.Recoltes en 1887 par M.A. Gaillard (suite). Bulletin de la Société Mycologique de France 4: 92-129.

Patouillard, N. \& Lagerheim, G. de. 1891. Champignons de l'Equateur. Bulletin de la Société Mycologique de France 7: 158-184.

Patouillard, N. \& Lagerheim, G. de. 1892. Champignons de l'Equateur (Pugillus II). Bulletin de la Société Mycologique de France 8: 113-140.

Patouillard, N. \& Lagerheim, G. de. 1893. Champignons de l'Equateur (Pugillus III). Bulletin de la Société Mycologique de France 9(2): 124-165.

Patouillard, N. \& Lagerheim, G. de. 1895a. Champignons de l'Equateur (Pugillus IV). Bulletin de l'Herbier Boissier 3: 53-74.

Patouillard, N. \& Lagerheim, G. de. 1895b. Champignons de l'Equateur (Pugillus V). Bulletin de la Société Mycologique de France 11: 205-234.

Pazschke, O. 1896. Verzeichnis brasilianischer von E. Ule gesammelter Pilze. Hedwigia 35: 50-55.

Pérez, J.M. \& Camino, M. 2000. Riqueza micológica en un sitio natural del Jardín Botánico Nacional. Revista del Jardín Botánico Nacional 21(1): 133-137.

Pérez-Moreno, J. \& Villarreal, L. 1988. Los hongos y myxomycetes del estado de Chiapas, Mexico. Estado actual de conocimiento y nuevos registros. Micología Neotropical Aplicada 1: 97-133.

Pérez-Silva, E. 1979. Primer registro del mixomycete Physarum flavicomum en Mexico. Boletín de la Sociedad Mexicana de Micología 13: 239-242.

Pérez-Silva, E. \& Aguirre-Acosta, E. 1985. Micoflora del Estado de Durango, Mexico. Revista Mexicana de Micología 1: 315. 329.

Pérez-Silva, E. \& Bárcenas, E. 1999. Nuevos registros de myxomycetes para el Estado de México. Ciencia Ergo Sum 6: 165-167.

Pérez-Silva, E., Herrera, T., Esqueda, M., Illana, C. \& Moreno, G. 2001. Myxomycetes of Sonora, Mexico I. Mycotaxon 77: 181-192.
Phillips, O. \& Miller, J.S. 2002. Global patterns of plant diversity: Alwyn H. Gentry's Forest transect data set. Monographs of Systematic Botany Missouri Botanical Garden 89.

Ponte, M.P.M.P., Cavalcanti, L.H. \& Mobin, M. 2003. Myxomycetes do Parque Zoobotânico de Teresina, Piauí, Brasil. Acta Botanica Brasilica 17: 1-18.

Pôrto, K.C. \& Cavalcanti, L.H. 1984. Myxomycetes da Floresta Estacional Perenifólia costeira (Recife - PE) I. Influência dos fatores climáticos. In: Congresso Nacional de Botânica, 1984, Porto Alegre. Anais. Porto Alegre.

Pôrto, K.C. \& Cavalcanti, L.H. 1986. Trichiaceae (Myxomycetes) da floresta pluvial tropical I. Arcyria Wiggers. $36^{\circ}$ Congresso Brasileiro de Botânica, 1986, Curitiba. Anais. pp. 867.

Pôrto, K.C., Cavalcanti, L.H. \& Correia, A.M.S. 1982. Incidência de Myxomycetes em Palmae. Anais XXXIII Congresso Nacional de Botânica, Maceió, 1982, pp. 181-187.

Putzke, J. 1996. Myxomycetes do Brasil. Cuadernos de Pesquisa, Série Botanica 8: 3- 133.

Putzke, J. 2002. Myxomycetes na Região Sul do Brasil. In: Araújo, E.L., Moura, A.N., Sampaio, E.V.S.B., Gestinari, L.M.S. \& Carneiro, J.M.T. (eds.), Biodiversidade, conservação e uso sustentável da flora do Brasil, pp. 221-223. Universidade Federal Rural de Pernambuco, Sociedade Botânica do Brasil, Recife.

Raunkiaer, C. 1928. Myxomycetes from the West Indian Islands St. Croix, St. Thomas and St. Jan. Dansk Botanisk. Arkiv 5(16): 1-9.

Real Jardin Botanico, Madrid: MA-Fungi (accessed through GBIF data portal, http://data.gbif.org/datasets/resource/1518, 200806-24).

Reid, D.A., Pegler, D.N. \& Spooner, B.M. 1981. An annotated list of the fungi of the Galapagos Islands. Kew Bulletin 35: 847-891.

Rodrigues, C.L.M. \& Guerrero, R.T. 1990. Myxomycetes do morro Santana, Porto Alegre, Rio Grande do Sul. Boletim do Instituto de Biociencias 46: 1-102.

Rodrigues, K.F. 1985. Contribuição ao estudo dos mixomicetos do Estado do Rio de Janeiro. Rodriguésia 37: 46-47.

Rodríguez, G. 1955. Adiciones a los Myxomycetes de Venezuela. Boletín del Museo de Ciencias Naturales 1(1): 83-88.

Rodríguez, G. 1957. Nuevas adiciones a los Myxomycetes de Venezuela. Acta Biológica Venezuelica 2(13): 135-138.

Rodríguez-Palma, M. 1998. Myxomycetes of the state of Tlaxcala. McIlvainea 13: 25-32.

Rodríguez-Palma, M. \& Estrada-Torres, A. 1996a. Some Stemonitales (Myxomycetes) from the state of Tlaxcala, Mexico. Mycotaxon. 60: 79-102.

Rodríguez-Palma, M. \& Estrada-Torres, A. 1996b. Distribution and biogeographic affinities of the Liceales community of the AbiesPinus forests from the Malintzi volcano, Mexico. In: Lado, C. \& Hernández, J.C. (eds.), Abstract Volume. Second International Congress on the Systematics and Ecology of Myxomycetes: 99.

Rodríguez-Palma, M., Estrada-Torres, A. \& Hernández-Cuevas, L. 2005. Myxomycetes (Protistas). In: Fernández, J.A. \& López, J.C. (eds.), Biodiversidad del Parque Nacional Malinche. Tlaxcala. Mexico.

Rodríguez-Palma, M., Lado, C. \& Estrada-Torres, A. 1996. Myxomycetes from a seasonal tropical forest in the Pacific coast of Mexico. In: Lado, C. \& Hernández, J.C. (eds.), Abstract Volume. Second International Congress on the Systematics and Ecology of Myxomycetes: 136.

Rodríguez-Palma, M., Varela-García, A. \& Lado, C. 2002. Corticolous Myxomycetes associated with four tree species in Mexico. Mycotaxon 81: 345-355.

Rogerson, C.T., Harris, R.C. \& Samuels, G.J. 1990. Fungi collected by Bassett Maguire and collaborators in the Guayana highland, 1944-1983. 
Rojas, C. \& Stephenson, S.L. 2007. Distribution and ecology of myxomycetes in the high-elevation oak forest of Cerro Bellavista, Costa Rica. Mycologia 99(4): 534-543.

Rojas, C. \& Stephenson, S.L. 2008. Myxomycete ecology along a elevation gradient on Cocos Island, Costa Rica. Fungal Diversity 29: 117-127.

Rorer, J.B. 1911. A preliminary list of Trinidad fungi. Dept. Agriculture Trinidad -Tobago Circ. 4: 37-44.

Rudolphi, F. 1829. Plantarum vel novarum vel minus cognitarum descripciones. Linnaea 4: 114-120.

Rufino, M.U.L. \& Cavalcanti, L.H. 2007. Alterations in the lignicolous myxomycete biota over two decades at the Dois Irmãos Ecologic State Reserve, Recife, Pernambuco, Brazil. Fungal Diversity 24: 159-171.

Rzedowski, J. 1991. El endemismo en la flora fanerogámica mexicana: una apreciación analítica preliminar. Acta Botánica Mexicana 15: 47-64.

Saccardo, P.A. 1892. Sylloge fungorum. Ed. Sumptibus auctoris typis Seminarii 10: 1-964.

Saccardo, P.A. 1895. Sylloge fungorum. Ed. Sumptibus auctoris typis Seminarii. 11: 1-753.

Saccardo, P.A. \& Saccardo, D. 1906. Sylloge fungorum. Ed. Sumptibus auctoris typis Seminarii 18: 208-214.

Saccardo, P.A. \& Sydow, P. 1899. Sylloge fungorum. Ed. Sumptibus auctoris typis Seminarii 14: 831-840.

Saccardo, P.A. \& Sydow, P. 1902. Sylloge fungorum. Ed. Sumptibus auctoris typis Seminarii 16: 819-824.

Saccardo, P.A. \& Trotter, A. 1913. Sylloge fungorum. Ed. Sumptibus auctoris typis Seminarii 22: 792-817.

Santos, E.J. \& Cavalcanti, L.H. 1988. Revisão de Myxomycetes ocorrentes em cana-de-açúcar (Saccharum spp.) no Brasil. Boletim de Micologia 4: 61-64.

Santos, E.J. \& Cavalcanti, L.H. 1991a. Adições à Mixoflora da Ilha de Maracá (Boa Vista-RR). Resumos da 15a. Reunião Nordestina de Botânica., Maceió, pp. 8.

Santos, E.J. \& Cavalcanti, L.H. 1991b. Myxomycetes do canavial I. Levantamento florístico em Carpina-PE. Acta Botanica Brasilica 5: 49-61.

Santos, E.J. \& Cavalcanti, L.H. 1995. Myxomycetes ocorrentes em bagaço de cana-de-açúcar armazenado em indústria. Boletim da Sociedade Broteriana 67: 5-22.

Santos, E.J., Cavalcanti, L.H. \& Albuquerque, W.C. 1986. Myxomycetes de Alagoas. In: Congresso Nacional de Botânica, 37. 1986, Ouro Preto. Anais. Ouro Preto (MG): Sociedade Botânica do Brasil, pp. 499-503.

Schinner, F. 1981. Myxomycetes from the tropical rain forest of Ecuador. Bericht des Naturwissenschaftlich-Medizinischen Vereins Innsbruck 68: 7-11.

Schnittler, M. 2001. Foliicolous liverworts as a microhabitat for Neotropical myxomycetes. Nova Hedwigia 72: 259-270.

Schnittler, M. \& Stephenson, S.L. 2000. Myxomycete biodiversity in four different forest types in Costa Rica. Mycologia 92: 626637.

Schnittler, M. \& Stephenson, S.L. 2002a. Inflorescences of Neotropical herbs as a newly discovered microhabitat for myxomycetes. Mycologia 94: 6-20.

Schnittler, M. \& Stephenson, S.L. 2002b. Myxomycetes from inflorescences of giant herbs and foliicolous liverworts. Scripta Botanica Belgica 22: 83.

Schnittler, M., Lado, C. \& Stephenson, S.L. 2002. Rapid biodiversity assessment of a tropical myxomycete assemblage - Maquipucuna Cloud Forest Reserve, Ecuador. Fungal Diversity 9: 135-167.
Seaver, F.J. \& Chardon, C.E. 1926. Scientific survey of Porto Rico and the Virgin Islands. New York Academy of Sciences Annual 8(1): 3-9.

Silva, M.I.L. \& Cavalcanti, L.H. 1988. Myxomycetes ocorrentes nos brejos de Pernambuco, I. Boletim de Micologia 4:31-35.

Spegazzini, C. 1880a. Fungi argentini. Pugillus primus. Anales de Sociedad Científica Argentina 9(4): 158-192.

Spegazzini, C. 1880b. Fungi argentini, Pugillus secundus (continuación). Anales de Sociedad Científica Argentina 10: 5-33.

Spegazzini, C. 1880c. Fungi argentini. Pugillus tertius. Anales de Sociedad Científica Argentina 10: 145-168.

Spegazzini, C. 1881. Fungi argentini. Pugillus quartus. Additis nonnullis Brasiliensibus Montevideensibusque. Anales de Sociedad Científica Argentina 12: 241-258.

Spegazzini, C. 1882. Fungi argentini additis non nullis Brasiliensibus Montevideensibus que. Revue Mycologique Tolouse 4(14): 121-123.

Spegazzini, C. 1886. Fungi guaranitici. Pugillus I. Anales de Sociedad Científica Argentina 22: 186-224.

Spegazzini, C. 1887a. Fungi patagonici. Boletín de la Academia Nacional de Ciencias. Córdoba 11(1): 5-64.

Spegazzini, C. 1887b. Fungi fuegiani. Boletín de la Academia Nacional de Ciencias. Córdoba 11: 135-308.

Spegazzini, C. 1888. Fungi guaranitici. Pugillus II. Anales de Sociedad Cientifica Argentina 26(1): 5-74.

Spegazzini, C. 1889. Fungi puiggariani. Boletín de la Academia Nacional de Ciencias. Córdoba 11:381-622.

Spegazzini, C. 1896a. Contribución al estudio de la flora de la Sierra de la Ventana [Fungi]. Min. Obras Publ. Buenos Aires.

Spegazzini, C. 1896b. Hongos de la caña de azúcar. Revista de la Facultad de Agronomía Veterinaria de La Plata 2(18): 227-258.

Spegazzini, C. 1899a. Mycetes argentinenses. Anales de Sociedad Cientifica Argentina 47(6): 262-279.

Spegazzini, C. 1899b. Fungi argentini novi v. critici. Anales del Museo Nacional de Historia Natural de Buenos Aires 6(3): 81-367.

Spegazzini, C. 1909a. Fungi in Ilice paraguariensi vigentes. Anales del Museo Nacional de Historia Natural de Buenos Aires 10: 114133.

Spegazzini, C. 1909b. Mycetes argentinenses. (Series.IV). Anales del Museo Nacional de Historia Natural de Buenos Aires 19: 257 458.

Spegazzini, C. 1912. Mycetes argentinenses. (Series.VI). Anales del Museo Nacional de Historia Natural de Buenos Aires 23: 1-146.

Spegazzini, C. 1913. Mycetes argentinenses. Anales del Museo Nacional de Historia Natural de Buenos Aires 24: 167-186.

Spegazzini, C. 1917. Algunos hongos chilenos. Revista chilena de Historia Natutal 21(4-5): 117-126.

Spegazzini, C. 1919a. Los hongos de Tucumán. I Reunión Nacional de la Sociedad Argentina de Ciencias Naturales 1916: 254-274.

Spegazzini, C. 1919b. Relquiae mycologicae tropicae et fungi Costaricensis nonnulli. Boletín de la Academia Nacional de Ciencias. Córdoba 23: 365-609.

Spegazzini, C. 1921. Mycetes chilenses. Boletín de la Academia Nacional de Ciencias. Córdoba 25: 1-124.

Spegazzini, C. 1923. Fungi paraguayenses. Anales del Museo Nacional de Historia Natural de Buenos Aires 31: 335-450.

Spegazzini, C. 1926. Algunas especies de Myxomycetas de la Argentina. Physis (Buenos Aires) 8: 417-419.

Spegazzini, C. 1927. Contribución al conocimiento de la flora micológica de las Sierras de Córdoba. Boletín de la Academia Nacional de Ciencias. Córdoba 29(3-4): 113-190.

Staatliche Naturwissenschaftliche Sammlungen Bayerns, The Myxomycetes Collections at the Botanische Staatssammlung 
München - Collection of Martin Schnittler (accessed through GBIF data portal, http: //data.gbif.org/datasets/resource /1444, 2008-06-25, and Collection of Hermann Neubert (accessed through GBIF data portal, http: //data.gbif.org /datasets/resource/1443, 2008-06-25)

Standley, P.C. 1927. The flora of Barro Colorado Island, Panama. Smithsonian Miscellaneous Collection 78(8): 417-419.

Standley, P.C. 1933. The flora of Barro Colorado Island, Panama. Contributions from the Arnold Arboretum 5: 5-159.

Stephenson, S.L. \& Mitchell, D. 1994. Notes on tropical Myxomycetes. I. Collections from Ecuador and Peru. Micología Neotropical Aplicada 7: 17-21.

Stephenson, S.L. \& Stempen, H., 1994. Myxomycetes A Handbook of Slime molds. Timber Press, Portland, Oregon.

Stephenson, S.L., Estrada-Torres, A., Schnittler, M., Lado, C., Wrigley, D. \& Ogata, N. 2003. Distribution and ecology of myxomycetes in the forests of Yucatan. In: Gómez-Pompa, A., Allen, M., Fedick, S. \& Jiménez-Osornio, J. (eds.), Lowland Maya Area: Three Millenia at the Human-Wildland Interface. Haworth Press, New York.

Stephenson, S.L., Landolt, J.C. \& Moore, D.L. 1999. Protostelids, dictyostelids, and myxomycetes in the litter microhabitat of the Luquillo Experimental Forest, Puerto Rico. Mycological Research 103: 209-214.

Stephenson, S.L., Schnittler, M. \& Lado, C. 2004a. Ecological characterization of a tropical myxomycete assemblage Maquipucuna Cloud Forest Reserve, Ecuador. Mycologia 96: 488-497.

Stephenson, S.L., Schnittler, M., Lado, C., Estrada-Torres, A., Wrigley de Basanta, D., Landolt, J., Novozhilov, Y.K., Clark, J., Moore, D.L. \& Spiegel, F.W. 2004b. Studies of neotropical mycetozoans. Systematics and Geography of Plants 74: 87-108.

Stevenson, J.A. 1971. An account of Fungus exsiccati containing material from the Americas. Nova Hedwigia 36: 1-564.

Stevenson, J.A. 1975. The fungi of Puerto Rico and the American Virgin Islands. Contributions from the Reed Herbarium 23: 1-743.

Stevenson, J.A. \& Cardenas, M. 1949. Lista preliminar de los hongos de Bolivia. Lilloa 21: 77-134.

Sturgis, W.C. 1916. Myxomycetes from South America. Mycologia 8(1):34-41.

Sydow, H. \& Sydow, P. 1907. Verzeichnis der von Hern F. Noack in Brasilien gesammelten Pilze. Annales Mycologici 5(4): 348363.

Toro, R.A. 1926. Mixomicetos de Santo Domingo. Imprenta de J.R. Vda. García. Santo Domingo. R. D.

Torrend, C. 1908. Les Myxomycètes. Étude des espèces connues jusqu'ici. Brotéria, Série Botanica 7: 5-177.

Torrend, C. 1915. Les Myxomycètes du Brésil, connus jusqu'ici. Brotéria 13(2): 72-88.

Torrend, C. 1916. Os Myxomycetes dos arredores da Bahia. In: Anon. (ed.), Anais do $5^{\circ}$ Congresso Brasileiro de Geographia. Sociedade Brasileira de Geographia, Salvador, pp. 484-492.

Trujillo-Flores, F. 1988. Contribución al conocimiento de los myxomycetes de la sierra de Manantlán, Jalisco. Tiempos de Ciencia 12: $20-27$

Trujillo-Flores, F., Castañeda Macías, M. \& Guzmán-Dávalos, L. 1986. Hongos del estado de Jalisco, VI. Los myxomycetes conocidos. Tiempos de Ciencia 5: 42-51.

University of Arkansas, Planetary Biodiversity Inventory Eumycetozoan Databank (accessed through GBIF data portal, http: //data.gbif.org/datasets/resource/1515, 2008-06-24).

Uribe-Meléndez, J. 1995. Catálogo de los Myxomycetes registrados para Colombia. Caldasia 18: 23-26.
Utah State University, USU-UTC Specimen Database (accessed through GBIF data portal, http: //data.gbif.org/datasets/ resource/1508, 2008-06-25).

Verde de Millán, L. \& Jaimes, F. 1987. Contribución al conocimiento de los Myxomycetes de Venezuela. I. Estado de Sucre. Boletín de la Sociedad Micológica de Madrid 11(2): 195-201.

Villarreal, L. 1983. Algunas especies de myxomycetes no registradas del Estado de Veracruz. Boletín de la Sociedad Mexicana de Micología 18: 153-164.

Villarreal, L. 1985. Nuevos registros de Myxomycetes en el Estado de Veracruz. Revista Mexicana de Micología 1: 363-378.

Villarreal, L. 1990. Estudios sobre los Myxomycetes de México, I. Nuevos registros. Micología Neotropical Aplicada 3: 67-79.

Welden, A.L. 1954. Some Myxomycetes from Panama and Costa Rica. Mycologia 46(2): 93-99.

Welden, A.L. \& Guzmán, G. 1978. Lista preliminar de los hongos, líquenes y myxomycetes de las regiones de Uxpanapa, Coatzacoalcos, Los Tuxtlax, Papaloapan y Xalapa (parte de los estados de Veracruz y Oaxaca). Boletín de la Sociedad Mexicana de Micología 12: 59-102.

Welden, A.L. \& Lemke, P.A. 1961. Notas sobre algunos hongos mexicanos. Boletín de la Sociedad Botánica Mexicana 26: 1-24.

Welden, A.L., Dávalos, L. \& Guzmán, G. 1979. Segunda lista de los hongos, líquenes y mixomicetos de las regiones de Uxpanapa, Coatzcoalcos, Los Tuxtlas, Papaolapan and Xalapa (Mexico). Boletín de la Sociedad Mexicana de Micología 13: 151-161.

Weston, W.H. Jr. 1933. The fungi of Barro Colorado. Scientific Monthly 36(5): 387-407.

Wheeler, Q.D. 1980. Studies on Neotropical slime mold / Beetle relationships, part I: Natura, history and description of a new species of Anisotoma from Panama (Coleoptera: Leiodidae). Proceedings of the Entomological Society of Washington 82(3): 493-498.

Wright, J.E. \& Albertó, E. 2006. Guía de los hongos de la Región Pampeana. II. Hongos sin laminillas. Ed. L.O.L.A., Buenos Aires.

Wrigley de Basanta, D. \& Lado, C. 2005. A taxonomic evaluation of the stipitate Licea species. Fungal Diversity 20: 261-314.

Wrigley de Basanta, D., Lado, C. \& Estrada-Torres, A. (in press). Description and culture of a new species of Didymium (Myxomycetes) from arid areas of Mexico. Mycologia

Wrigley de Basanta, D. \& Stephenson, S.L. 2005. Mycetozoan Biodiversity in the realm of the condor. Inoculum 56 (2): 5-6.

Wrigley de Basanta, D., Estrada-Torres, A. \& Lado, C. 2003. Biodiversity Surveys for Neotropical Mycetozoans in Cuba and Mexico. Inoculum 54(1): 1-2.

Wrigley de Basanta, D., Lado, C., Stephenson, S.L. \& EstradaTorres, A. 2002. Myxomycetes from moist chamber cultures of Neotropical substrates. Scripta Botanica Belgica 22: 100.

Wrigley de Basanta, D., Stephenson, S.L., Lado, C., Estrada-Torres, A. \& Nieves-Rivera, A.M. 2008. Lianas as a microhabitat for myxomycetes in tropical forests. Fungal Diversity 28: 109-125.

Yamamoto, Y. 2000. Several Mexican Myxomycetes preserved in TNS. The Myxomycetes 18: 43-45.

Yamamoto, Y., Hagiwara, H. \& Kawakami, S. 2000. Brazilian Myxomycetes in the Herbarium of the National Science Museum, Tokyo. Bulletin of the National Science Museum. Tokyo, Ser. B 26(4): 123-133.

Associate Editor: F. Pando Received: $27-\mathrm{V}-2008$ Accepted: 7-VII-2008 